\title{
Target the ego or target the group: Evidence from a randomized experiment in proactive churn management
}

\author{
Miguel Godinho de Matos \\ Católica Lisbon School of Business and Economics,miguel.godinhomatos@clsbe.lisboa.ucp.pt \\ Pedro Ferreira \\ Heinz College and Department of Engineering and Public Policy, Carnegie Mellon University, pedrof@cmu.edu \\ Rodrigo Belo \\ Rotterdam School of Management, Erasmus University, rbelo@rsm.nl
}

\begin{abstract}
We propose a new strategy for proactive churn management that actively uses social network information to help retain consumers. We collaborate with a major telecommunications provider to design, deploy and analyze the outcomes of a randomized control trial at the household level to evaluate the effectiveness of this strategy. A random subset of likely churners were selected to be called by the firm. We also randomly selected whether their friends would be called. We find that listing likely churners to be called reduced their propensity to churn by 1.9 percentage points from a baseline of $17.2 \%$. When their friends were also listed to be called their likelihood of churn reduced an additional 1.3 percentage points. The NPV of likely churners increased $2.1 \%$ with traditional proactive churn management and this statistic becomes $6.4 \%$ when their friends were also listed to be called by the firm. We show that in our setting likely churners receive a signal from their friends that reduces churn among the former. We also discuss how this signal may trigger mechanisms akin to both financial comparisons and conformity that may explain our findings.
\end{abstract}

Key words: Proactive Churn Management, Social Networks, Randomized Experiment

\section{Introduction}

Customer retention is a central concern in many industries, in particular in IT-related markets, where churn rates are considerably high. For example, cell phone companies report churn rates as high as $2 \%$ per month (Bensoussan et al. 2014) and pay-TV firms experience churn rates of about $1 \%$ per month (Green 2016). Such high churn rates have a significant impact on the value and profitability of firms (Gupta et al. 2004). As a consequence, customer retention is a fundamental issue in marketing research. In general, there are two main approaches to manage customer churn: firms can either wait for consumers to request service to be disconnected and extend them aggressive retention deals when this happens - reactive churn management - or they can try to anticipate which consumers are likely to churn and reach out to them before they do - proactive churn management. Reactive churn management focuses on users that have already signaled their 
willingness to leave the firm. However, at that stage, it might be too late to convince them to stay. With proactive churn management, firms reach consumers sooner, which may be cheaper, but they may also extend deals to users that were unlikely to leave.

Proactive churn management is often viewed as a more sophisticated approach potentially leading to better results for the firm. However, it is still hard to generalize the effects of proactive churn management even within the telecommunications industry. While some studies found that proactive churn management helps retain consumers, such as Burez and Van den Poel (2007) in the context of pay-TV services, others report an increase in the churn rate of the targeted consumers, such as Ascarza et al. (2016b) in the context of prepaid mobile. In parallel, a number of studies have looked at the role social networks in consumer decision making in the context of IT. However, only a few studies have looked at the impact of peer effects on churn. Nitzan and Libai (2011) analyzed a large dataset from a cell phone provider and found that exposure to a churner is positively associated with churn. In a more recent study, Ascarza et al. (2016a) identified customers at risk in another cell phone provider and found that giving them a monetary credit increased usage and lowered churn across both the treated consumers and their connections. The authors attribute this result to the explicit network externalities associated to how consumers can use the gifted credit to place calls.

In this paper we study a new strategy to retain consumers and compare its performance to that of traditional proactive churn management. Once proactive churn management may be profitable in pay-TV markets and IT-related services may benefit from peer influence, we ask whether it may be profitable for a telecommunications firm that offers triple-play service to combine churn management with peer effects. In particular, and in a context without explicit network externalities, does it help to reach out to the friends of likely churners as a way to create "goodwill" around the latter to retain them? The contribution of our paper relies on actively using the social network to design a profitable intervention for the firm as opposed to the previous literature that only used the social network to find peer effects a-posteriori. We call this new strategy, in which the friends of likely churners are also contacted by the firm, socially-based proactive churn management. This strategy proposes a "group-centric" approach to manage churn while traditional proactive churn management is usually focused on targeting consumers independently.

We collaborate with a major telecommunications provider to design, implement and analyze outcomes of a randomized control trial at the household level. We use data from pay-TV subscriptions to develop a model to predict likely churners, and data from Call Detailed Records (CDRs) to draw a social graph across households. On a monthly basis, and during 8 consecutive months, we select a random set of likely churners and their friends and allocated them randomly across four different experimental conditions. In particular, we randomly, and independently, select whether 
both the former and the latter are listed to be called by firm's call center. The goal of these calls is to identify likely churners and extend them retention deals to demote them from churning.

We find that in our setting traditional proactive churn management decreases the churn rate of likely churners by 1.9 percentage points $(p-$ value $<0.01)$, from a baseline of $17.2 \%$. The latter is the churn rate observed with reactive retention only. We also find that socially-based proactive churn management decreases this statistic by an additional 1.3 percentage points $(p-v a l u e<0.05)$ and increases customer lifetime value relative to traditional proactive churn management, thus improving the firm's bottomline. In addition, we also find no significant change in the churn rate of the friends of likely churners across all our treatment conditions. Our results show that in our setting likely churners receive a signal from their friends that lowers their likelihood of churn, which the firm can use to its benefit by targeting consumers alongside with their friends. This signal may trigger mechanisms akin to conformity and financial comparisons, which are likely to explain the results we observe in our setting. The remainder of this paper is organized as follows. Section 2 reviews the relevant literature. Section 3 describes the context of our study and section 4 explains our experiment in detail. Section 5 shows our results and section 6 concludes.

\section{Relevant Background}

Customer retention has been a central issue in marketing research since the late 1990s. The early studies focused on identifying the drivers of consumer churn and highlighted that customer demographics, such as age, gender and tenure, satisfaction, and perception of fairness affect the length of stay (e.g., Bolton 1998, Bolton and Lemon 1999). Customer retention has been studied in many different contexts, such as loyalty programs, switching costs, customer self-service channels, pricing policies and advertising expenditure, management of firm-customer interactions (Reinartz and Kumar 2003, Venkatesan and Kumar 2004, Reinartz et al. 2005). In general, these studies show that the drivers of customer churn are similar across industries and over time. Customer retention has also become a fundamental concern for practitioners since it has been directly linked to firm profitability (Anderson et al. 1994, Rust et al. 2004, Villanueva et al. 2008). Even small increases in retention rates have been shown to yield significant increases in profits (Reichheld and Sasser 1990, Reichheld 2003) because existing consumers tend to be more loyal and generate more revenue (Reichheld and Sasser 1990, Reichheld 2003).

Firms pursue two main approaches to manage customer churn (Winer 2001), namely reactive churn management, whereby they wait for consumers to signal their intention to leave and at that time they try to avoid so by offering them aggressive retention deals, and proactive churn management, whereby firms try to contact likely churners early to extend them deals to retain them. Proactive churn management may yield higher profits because firms reach consumers at 
a point in time at which they are cheaper to retain, for example, before contracts expire when consumers have not yet surveyed the market to learn about alternatives. However, the firm needs to identify customers at risk to implement proactive churn management. In particular, the firm needs to reach true churners to avoid losing too much profit with false positives. Therefore, proactive churn management typically starts by identifying likely churners, which has been an exercise that attracted significant research effort in the churn management literature. Neslin et al. (2006) and Lemmens and Croux (2006) are among the first to study data-intensive algorithms to predict churn. Please refer to Ascarza et al. (2017) for a detailed review of more recent papers on this topic.

The traditional approach to proactive churn management suggests that firms should target individuals with high probability of churn. (Blattberg et al. 2008, pp. 632). However, the literature provides mixed results in this respect. In the context of pay-TV, Burez and Van den Poel (2007) find a positive effect of proactive churn management on profit. The authors study churn using data from a company that experiences a churn rate of $15 \%$ /year, select the top $30 \%$ likely churners, and gift them with an invitation to a unique event, free movie tickets or simply ask them to fill in a satisfaction survey. All treatment conditions lead to significant lower churn rate (reductions between $3 \%$ and $5 \%$ from the statistic above), with the latter condition being the most efficient in terms of profit to the firm given its low implementation cost (essentially, not extending offers to consumers). In contrast, Ascarza et al. (2016b) study churn in a market for prepaid mobile service - experiencing a $6 \%$ average churn rate per quarter - and find a negative effect of proactive churn management. The authors select users predicted to benefit from upgrades in their tariff plan and offer a random subset of them a $\$ 15$ credit for a period of 3 months, conditional on them upgrading their tariff plan. They find that the churn rate across treated consumers increases to 10\%/quarter. The authors provide additional evidence showing that the intervention might have reduced the customers' inertia to change and increased the saliency of usage, potentially leading some customers to search for services from competitors that could fit better their calling needs.

These studies pertain to very different contexts. The former looks at a market where consumers become locked-in before they can churn without paying a financial penalty. The latter looks at a pre-paid service from which consumers could churn by simply winding down their account balance. These differences alone may account for the different churn rates observed in these markets, the differences in the observed behavior of consumers and the differences in the mechanisms that led to such behavior, but they highlight that the question of who to target is far from trivial. More recently, a number of studies propose to choose targets based on their expected profitability (Lemmens and Gupta 2017) or on their likelihood of changing behavior if targeted (Ascarza 2017), rather than on their absolute level of risk, because such approaches are likely to better maximize client lifetime value (Gupta et al. 2004, Blattberg et al. 2008). The argument used in these papers 
is that optimizing targeting algorithms only to identify the individuals that are most likely to churn may end up targeting less profitable ones, i.e., those that would always stay with the firm, those that never stay with the firm or those that may be induced to leave due to targeting.

All studies mentioned before assume that consumers make independent decisions about which services to abandon. In these studies, consumers are described as independent agents that make decisions based on their individual experience with the services that they subscribe, their personal profile and the marketing they receive (Peres et al. 2010, Solomon 2014). Accordingly, marketing campaigns have been largely designed to target consumers one by one. However, in recent times, researchers began collecting evidence showing that social networks play an important role in many consumer decisions including churn. Nitzan and Libai (2011) run an observational study over 1 million customers of a cellular company and find that exposure to a churner is associated to an increase of $80 \%$ on the hazard of churn. The authors find this result after controlling for a number of individual level covariates that proxy social, personal and purchase-related traits, which lessens concerns with endogeneity. They also discuss how this effect is economically large, by comparing it to those found in the literation on adoption. In addition, they also find that the effect of social influence depends on number of ties, tie strength, customer loyalty and decays exponentially over time. Highly connected customers are more affected by neighbor defection and loyal customers are less so. This study provides a thorough analysis of how communication across consumers affects churn and shows why studying the relationship between peer effects and churn is paramount for managers in the field.

Ascarza et al. (2016a) complements our knowledge of peer influence in churn. The authors report the existence of spillovers in churn behavior by looking at the outcomes of a randomized field experiment in prepaid mobile. They find that proactively targeting likely churners has a positive effect not only on the targeted individuals but also on their friends. The authors identify customers at risk as those that would have their accounts suspended if they did not top up their balance in one week. They sample 961 such customers and treat $67 \%$ of them, selected at random, with a credit incentive that could be used to place calls. The authors find that their intervention increases usage by $35 \%$ on the targeted consumers and $10 \%$ on the neighbors of the targeted consumers. They also find that the friends of targeted consumers churn less, a spillover effect that they attribute to the nature of the product studied. Their setting is one with explicit network externalities whereby consumers can place an unlimited number of in-network calls and use their balance to pay for outnetwork and international calls. Therefore, in their setting peer influence was likely to be triggered by this economic principle.

Our paper contributes to both these lines of research by testing whether peer effects can be exploited to improve the performance of proactive churn management. We propose that firms 
can actively use the social network to design profitable interventions as opposed to the previous literature that only used the social network to find peer effects a-posteriori. In particular, we test whether targeting likely churners and their friends in the same proactive retention campaign with the same menu of non-referral retention deals reduces churn. Unlike Nitzan and Libai (2011) and Ascarza et al. (2016a), we study a context where the way consumers use the service does not involve explicit network externalities, and thus it is unclear whether peer effects would arise in such a setting and, if so, whether they would be economically significant. Finally, and in line with the suggestions in (Reichheld and Sasser 1990, Berger and Nasr 1998, Jain and Singh 2002, Rust et al. 2004), we use customer lifetime value to compare the performance of our strategy to that of traditional proactive churn management, thus measuring its effect on the firm's bottomline.

\section{Context and Historical Data Sources}

We study whether the peer effects in churn decisions discussed in Nitzan and Libai (2011) and in Ascarza et al. (2016a) in cell phone markets may also arise in the context of triple play markets without explicit network externalities and, if so, whether firms can use them to improve churn management. Therefore, our setting is closer in nature to that studied in Burez and Van den Poel (2007) but these authors did not analyze peer effects. The relevance of studying triple-play markets, as opposed to cell phone service and pay-TV service separately, stems from the fact that triple-play is now becoming the dominant mode of consumption for telecommunications services. According to Digital TV Research, global triple-play subscriptions will reach 400 million by the end of 2017, up by nearly 300 million from the end of 2011. In addition, firms experience churn rates as high as $1 \%$ per month in these markets (Green 2016).

We collaborate with a large multinational telecommunications provider, hereinafter called TELCO. In the geography we analyze, TELCO serves more than 1 million households with TV, Internet and telephony. In this setting, households become locked-in when they contract service. We have anonymized data from TELCO between January 2013 and March 2015 and then for the month of January 2016. These data contain monthly snapshots of all TELCO products subscribed by all TELCO households. We also know each household's monthly bill and the price charged by competitors for similar services in the same region. In April date 2014, the average monthly bill at TELCO was $\$ 54.7 /$ month. Therefore, choosing whether to stay with TELCO or churn is a decision that has significant financial implications for consumers in our setting.

We also have anonymized Call Detailed Records (CDRs) for all landline calls served by TELCO between July 2012 and June 2013. This dataset contains over 600 million records. We use these data to define an undirected graph of communications across households. We build this graph by matching all anonymized phone numbers to their corresponding anonymized pay-TV accounts. 
We discard all CDRs where one of the calling parties is a number with no counterpart in the anonymized pay-TV database. An edge between two households is included in this graph if all of the following conditions are met: (1) there are two or more calls between them; (2) at least one of the calls took place after 7pm; (3) at least one of the calls occurred during the weekend; (4) there are calls between them in at least two months. Our criteria to add edges to this graph ensure that households contacted each other over landlines, after normal working hours, on weekends, and more than once. Thus, edges in our graph proxy real proximity between households and possibly family ties. The resulting social graph contains 1.2 million nodes and 2.63 million edges. The average and the median degree are 4.3 and 3, respectively. The household in 99th percentile is connected to 20 households.

\section{Our Experiment}

The best way to determine whether proactive churn management may help TELCO retain customers is to implement a randomized control trial in which a random set of likely churners are listed to be called by the firm and another random set of likely churners is hold out for control purposes. This setup allows us to control for all unobserved effects that may lead likely churners to churn and that could otherwise be related to proactive churn management had the firm selectively chosen households to call. In addition, we are also interested in learning whether the social network can be used to increase the efficiency of proactive churn management by leveraging potential peer effects. However, many unobserved factors that lead households to churn from TELCO are likely to be correlated among households connected in the social graph due to homophily. In fact, it is well known that empirically measuring the effect of peer influence is a hard task due to the confounding effects introduced by unobserved covariates that simultaneously drive friendship and behavior (Manski 1993, McPherson et al. 2001, Shalizi and Thomas 2011). We use a carefully designed randomized experiment to measure peer effects in our setting as described below. Our experiment is inspired in what has been done in the previous literature in other settings, such as Facebook (Aral and Walker 2011), news aggregators (Muchnik et al. 2013) and music recommendations (Bapna and Umyarov 2015).

\subsection{Initial Setup}

The initial setup of our experiment took place in the end of April 2014 and consisted in developing a model to predict churn. Using the monthly panel of households served by TELCO, we developed a model that maps a vector of household characteristics to likelihood of churn. This model was constructed using state-of-the-art data mining algorithms for classification tasks. Its details, training parameters, cross validation and performance metrics are all presented in Appendix B. This churn prediction model shows that tenure, an active lock-in period, the amount billed, the age of the 
contract holder and how much the household is off-price are among the most important predictors of churn at TELCO. How much the household is off-price is measured by the difference between her monthly bill and the lowest monthly bill offered by competitors for a similar bundle of services in the same region. Building this churn prediction model implements step one in the framework proposed by Blattberg et al. (2008) to measure the effect of proactive churn management. This churn prediction model allows us to focus our experiment on likely churners. As a consequence, our results generalize only to TELCO's likely churners, which is the subpopulation of interest for churn management purposes.

In addition, and also in April of 2014, we identified the ego-network of each household in our social graph. In social network analysis, the ego-network of a node in a social graph is the subgraph containing that node - called ego - all nodes connected directly to it - called alters - and all the edges between them (Wasserman 1994). Panel (1) in Figure 1 shows an example of an ego network. Finally, and also in April 2014, we also determined the sample size required to identify an effect that would render proactive churn management profitable on egos. Finding an effect that does not generate profit would be uninteresting from an economic point of view. Appendix $\mathrm{C}$ provides the details of the calculations that we performed for this purpose, which allowed us to determine that our experiment should run for at least 8 consecutive months. 

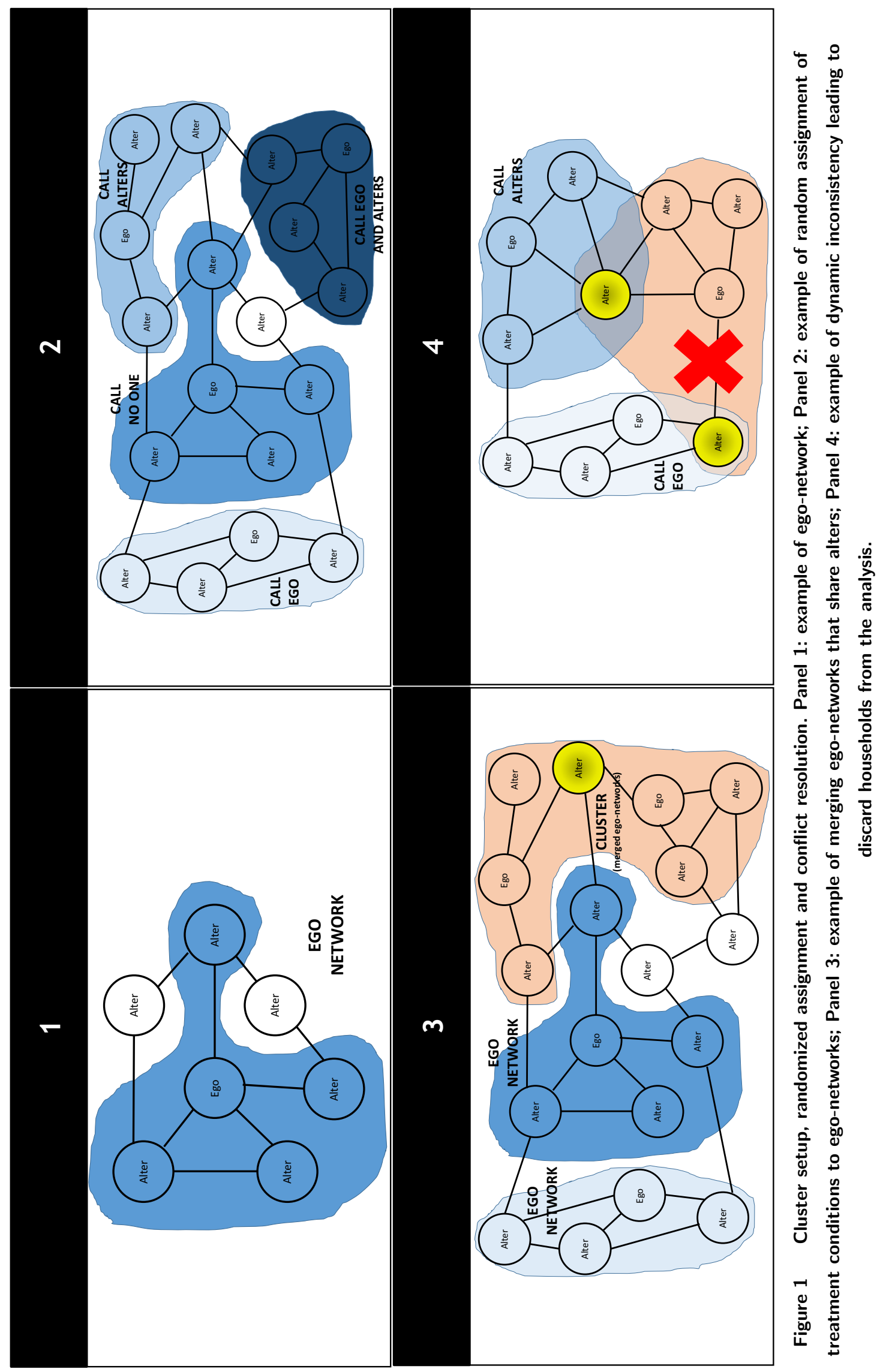


\subsection{Monthly Operations}

Every month starting in May 2014 TELCO provided us access to a dataset with a collection of covariates that allowed us to determine whether a household initiates churn. Households can initiate churn through several channels - by phone, mail, email or visiting a TELCO store. A household that performs any of these actions to churn is marked as so in our dataset. Every month we run our churn prediction model for households that did not initiate churn in the previous three months. TELCO refers to this period as "mourning". Its purpose is to ensure that households do not receive multiple contacts when parallel negotiations may already be underway. We note that our churn prediction model was retrained twice during our experiment, namely in May and October 2014, when TELCO refreshed our datasets on household level covariates. Table 1 shows a timeline of our experiment and which data was used when for what purpose.

Table 1 Experimental timeline indicating the calibration of our churn prediction model and the data used to select households into the experiment.

\begin{tabular}{|l|l|}
\hline \multicolumn{2}{|c|}{ Churn prediction model } \\
\hline Training and calibration & Used to select households \\
\hline \hline $\begin{array}{l}\text { Trained in Apr-2014 using data up to Dec-2013 } \\
\text { To predict churn Jan-15 to Feb-15 2014 }\end{array}$ & \\
\hline & $\begin{array}{l}\text { Used in May-2014 with data up to Apr-2014 } \\
\text { To predict churn Jun-15 to Jul-15 2014 } \\
\text { Used to select 1st wave of households on Jun 1st 2014 }\end{array}$ \\
\hline $\begin{array}{l}\text { Retrained in Jun-2014 using data up to Feb-2014 } \\
\text { To predict churn Mar-15 to Apr-15 2014 }\end{array}$ & \\
\hline & $\begin{array}{l}\text { Used in Jun-2014 with data up to May-2014 } \\
\text { To predict churn Jul-15 to Ago-15 2014 } \\
\text { Used to select 2nd wave of households on Jul 1st 2014 }\end{array}$ \\
\hline & $\begin{array}{l}\text { Used in Jul-2014 with data up to Jun-2014 } \\
\text { To predict churn Ago-15 to Sep-15 2014 } \\
\text { Used to select 3rd wave of households on Ago 1st 2014 }\end{array}$ \\
\hline & $\begin{array}{l}\text { Used in Ago-2014 with data up to Jul-2014 } \\
\text { To predict churn Sep-15 to Oct-15 2014 } \\
\text { Used to select 4th wave of households on Sep 1st 2014 }\end{array}$ \\
\hline & $\begin{array}{l}\text { Used in Sep-2014 with data up to Ago-2014 } \\
\text { To predict churn Oct-15 to Nov-15 2014 } \\
\text { Used to select 5th wave of households on Oct 1st 2014 }\end{array}$ \\
\hline $\begin{array}{l}\text { Retrained in Oct-2014 using data up to May-2014 } \\
\text { To predict churn Jun-15 to Jul-15 2014 }\end{array}$ & $\begin{array}{l}\text { Used in Oct-2014 with data up to Sep-2014 } \\
\text { To predict churn Nov-15 to Dec-15 2014 } \\
\text { Used to select 6th wave of households on Nov 1st 2014 }\end{array}$ \\
\hline & $\begin{array}{l}\text { Used in Nov-2014 with data up to Oct-2014 } \\
\text { To predict churn Dec-15 2014 to Jan-15 2015 } \\
\text { Used to select 7th wave of households on Dec 1st 2014 }\end{array}$ \\
\hline & $\begin{array}{l}\text { Used in Dec-2014 with data up to Nov-2014 } \\
\text { To predict churn Jan-15 to Feb-15 2015 }\end{array}$ \\
Used to select 8th wave of households on Jan 1st 2015 \\
\hline
\end{tabular}

Each month during the experiment we randomly placed the ego-network of each top (as defined in appendix C) likely churner in one of four conditions: Call No One - the ego and her alters are all listed not to be called by the firm; Call Ego - the ego is listed to be called by the firm and all 
her alters are listed not to be called by the firm; Call Alters - the ego is listed not to be called by the firm and all her alters are listed to be called by the firm; and Call Ego and Alters - both the ego and all her alters are listed to be called by the firm. We choose to list either no alter or all alters to be called by the firm to maximize our chance of finding an effect of peer influence. If there is no effect when all alters are listed to be called by the firm then it is very unlikely that an effect arises when fewer alters are listed to be called.

Panel (2) in Figure 1 shows an example of a network in which each ego-network is randomly placed in one of these four different conditions. This panel in this figure shows how considering ego-networks introduces separation between egos in different treatment conditions, which reduces the likelihood of violating the Stable Unit Treatment Value Assumption (SUTVA) (Wooldridge 2010, Rosenbaum 2007). Violating SUTVA would hamper our ability to identify causal treatment effects due to interference across conditions. This is a common concern in network settings (e.g., Manski 1993, McPherson et al. 2001, Shalizi and Thomas 2011, Eckles et al. 2014). Randomizing treatment assignment to ego-networks addresses this concern as suggested in Ugander et al. (2013). Considering ego-networks in our setting ensures that egos in different treatment conditions are at least two hops away from each other. Assuming only one-hop peer influence is a common practice in the literature in peer effects (e.g., Aral and Walker 2011, Bapna and Umyarov 2015, Ugander et al. 2012). In our setting, only $2.8 \%$ of the egos are connect to egos in a different cluster by two hops. Moreover, less than $2 \%$ of the egos are connected by two hops to egos in clusters with different treatment conditions. Furthermore, we find no evidence of peer influence over two-hops in our case (results available upon request).

Complex situations arise when ego networks overlap such as in panel (3) of Figure 1, where two egos share one alter. In order to ensure consistency, in this case, we merge the two ego-networks into a larger cluster, assign the same condition to both egos in this cluster and assign the same condition to all alters in the cluster. Therefore, alters in this cluster will either all be listed to be called by the firm or all be listed not to be called by the firm. In other words, once clusters are formed (and without overlap, clusters are just ego-networks), each cluster is randomly placed in one of the four treatment conditions referred above. Finally, we note that our experiment took place over several months. Therefore, when we select an ego to be included in the experiment it may be the case that it is connected to households that were already assigned to different treatment conditions in previous time periods. According to our experimental design this would require us to merge clusters that were assigned to different treatment conditions, such as in panel (4) in Figure 1. We circumvent this limitation of our experimental design by discarding from our analysis all households in the new cluster. This limitation led us to discard only $1 \%$ of the households in our sample. 
After the experimental procedure described above, the monthly random assignment of clusters to our four treatment conditions resulted in lists of households to be called by the firm and lists of households not to be called by the firm that we provided to TELCO's call center every month. We note that in our setting reactive retention is modeled by the condition Call No One, under which households still churn organically by calling the firm. Our other three treatment conditions embody an element of proactive churn management given that the firm is the party initiating contact. We followed households for a period of 120 days after treatment assignment and again in January 2016, that is, one year after our experiment ended. We note that from historical data, $95 \%$ of true positives listed by our predictive model request to disconnect their service (that is, enter the reactive retention) in the first 120 days after being identified by our model.

\subsection{Descriptive Statistics and Balance Across Treatment Conditions}

Figure 2 plots the number of clusters in each treatment condition. There are many more clusters in the Call Ego condition because TELCO required us to focus its call center efforts on likely churners. In fact, TELCO increased the capacity of the call center allocated to our experiment one month after it started and added more slots to call likely churners. This did not raise problems to our experiment because, overall, we still listed enough households to call in each and every treatment condition to identify the desired effects. Table 2 provides precise definitions for all covariates used throughout our paper. Tables 3,4 and 5 provide descriptive statistics for the most important covariates that in our setting shape consumer behavior - churn score, monthly bill and lock-in period - for all egos, all alters and all clusters in our experiment, respectively. The average degree of egos and alters in our experiment is 1.84 and 6.36, respectively. Their average churn scores are 0.71 and 0.24 , respectively. As expected, the churn score of egos is much higher in our setting. The average monthly bill and lock-in period for egos 30 days before treatment assignment were $\$ 56.2$ and 31.1 days, respectively. These statistics are $\$ 54.7$ and 132.2 for alters, respectively. Therefore, in our setting, alters are much less inclined to churn because they enjoy lower monthly bills and face longer lock-in periods. Appendix D provides additional evidence of balance in these covariates across treatment conditions on a monthly basis.

\subsection{Offers, Potential Outcomes and Compliance Levels}

Figure 3 describes the decision stages and possible outcomes for households included in the experiment. At the start of each month, lists of households to be included in the experiment were sent to the call center. Some of these households, selected at random as described in Section 4.2, were marked to be called by the call center dialler. The remaining households were marked not to be called, which helped ensure compliance with the hold out conditions. The latter households do not change their service conditions, unless they call the firm to leave, in which case they enter reactive 


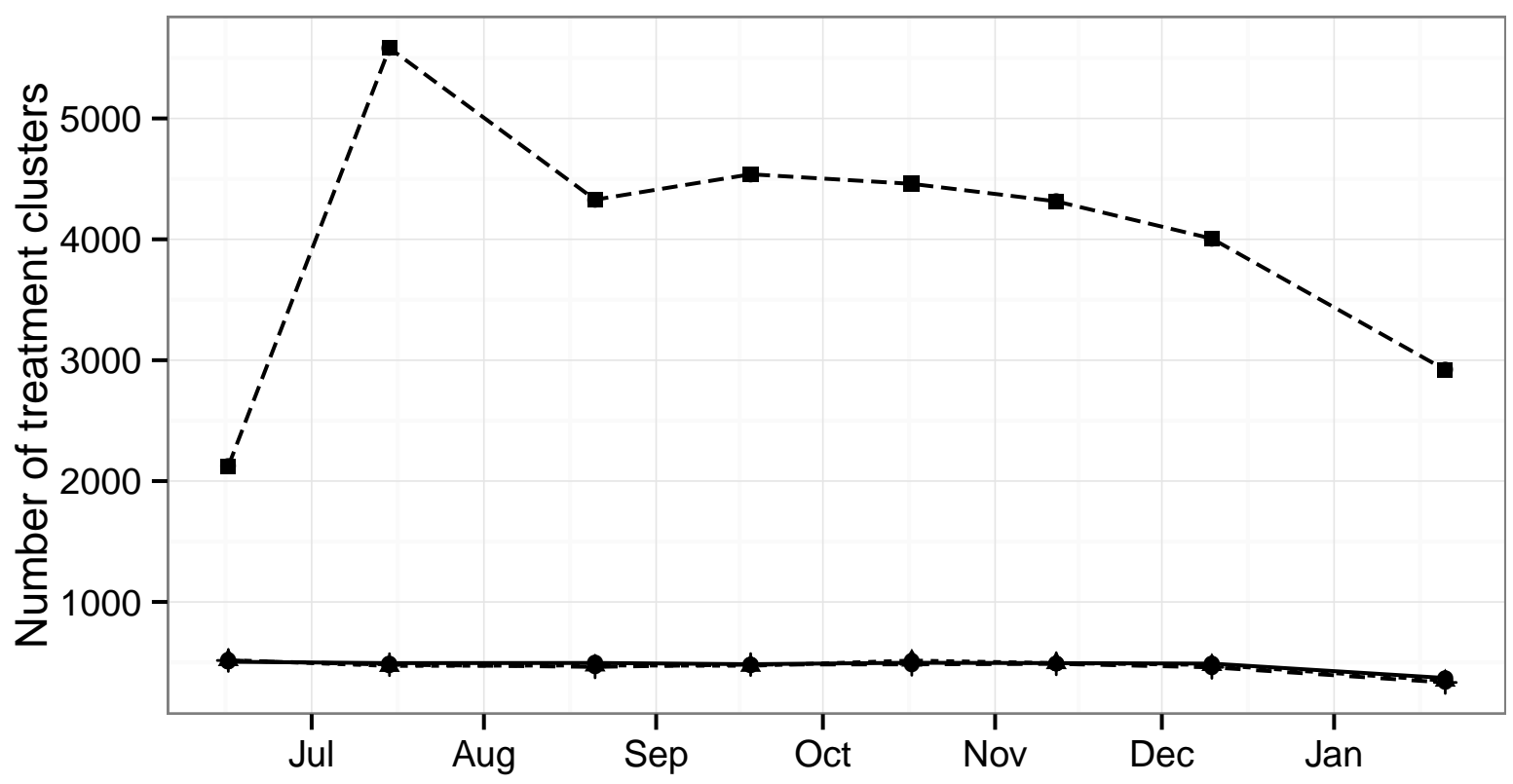

$\rightarrow$ Call No One -^. Call Alter -\#. Call Ego + Call Ego and Alter

Figure 2 Number of clusters per treatment condition over time during the experiment.

retention. Call center operators were not told which households were egos nor alters. They did not know the churn scores from our churn prediction model and they were not told that some households in these lists were connected in the social graph. The operations of TELCO's call center are based on automatic dialers that randomize the order and time at which households are contacted and recontacted when previous calls fail. In short, in our setting, there is no selection bias in terms of how calls were attempted. Yet, a number of unknown reasons may result in missed calls. It is still up to each household to pick up the phone from TELCO. This introduces a first layer of selection in our experiment. The top-left and top-right panels in Figure 4 show that egos listed to be called by the firm were reached $51 \%$ of the time and that alters listed to be called by the firm were reached $57 \%$ of the time.

When a call is established the call center operator tries to assess whether the household reached is likely to churn. A satisfaction survey is used to do so. All call center operators follow the same survey, i.e., the same questions in the same order were asked during each and every call made. If the call center operator determines that the household is unlikely to churn she explains why the current contract and service fit the needs of that household and wraps up the call with no offer and thus there is no change in the household's contractual conditions. If, on the other hand, the 
Table 2 Definition of variables used in the paper.

\begin{tabular}{|c|c|c|}
\hline Name & Type & Description \\
\hline Call No One & $\begin{array}{l}\text { Dummy } \\
\text { Variable }\end{array}$ & $\begin{array}{l}=1 \text { for households in clusters assigned to the Call No One } \\
\text { condition }\end{array}$ \\
\hline Câll'Älters & Dummy & 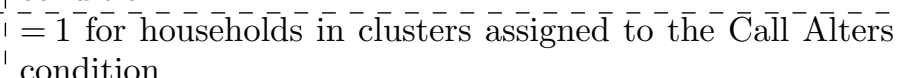 \\
\hline C̄all' Egō & $\begin{array}{l}\text { Dummy } \\
\text { Variable }\end{array}$ & $\begin{array}{l}=1^{-} \text {for households in clusters assigned to the }{ }^{-} \bar{C}^{-}{ }^{-}{ }^{-} \bar{E} \text { 'o } \\
\text { condition }\end{array}$ \\
\hline Cáll Ego and Álters & $\begin{array}{l}\text { Dummy } \\
\text { Variable }\end{array}$ & $\begin{array}{l}=1 \text { for households in clusters assigned to the call Ego and } \\
\text { Alters condition }\end{array}$ \\
\hline Churn Score & $\begin{array}{l}\text { Continuous } \\
\text { Variable }\end{array}$ & 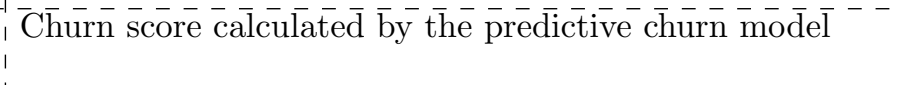 \\
\hline$\overline{\mathrm{R}} \overline{\mathrm{R}} \overline{\mathrm{A}} \overline{\mathrm{fter}} \overline{1} \overline{2} 0 \overline{\mathrm{D}} \overline{\mathrm{D}}-\overline{\mathrm{s}}$ & $\begin{array}{l}\text { Dummy } \\
\text { Variable }\end{array}$ & $\begin{array}{l}=1 \text { for households that request to churn within } \overline{1} 2 \overline{0} \text { after } \\
\text { treatment assignment }\end{array}$ \\
\hline Churn by Jan 2016 & $\begin{array}{l}\text { Dummy } \\
\text { Variable }\end{array}$ & $=1$ for households that churned by January $20 \overline{1} 6$ \\
\hline$\overline{\text { Lock-in Period }}-\overline{1}$ & $\begin{array}{l}\text { Continuous } \\
\text { Variable }\end{array}$ & $\begin{array}{l}\text { Number of days to current contact expiry } \overline{1} \overline{2} 0 \text { days before } \\
\text { treatment assignment }\end{array}$ \\
\hline Monthly $\overline{\text { Bill }}$ & $\begin{array}{l}\text { Continuous } \\
\text { Variable }\end{array}$ & $\begin{array}{l}\text { Monthly bill associated to all telecommunication services } \\
(=0 \text { for households who churn) }\end{array}$ \\
\hline Géts Câll & $\begin{array}{l}\text { Dummy } \\
\text { Variable }\end{array}$ & $=1$ for households that got a call from the call center ${ }^{-}{ }^{-}-$ \\
\hline & $\begin{array}{l}\text { Continuous- } \\
\text { Variable }\end{array}$ & $\begin{array}{l}\text { Proportion of household's friends that got a call from the } \\
\text { call center }\end{array}$ \\
\hline$\overline{\mathrm{G}} \overline{\mathrm{et}} \overline{\mathrm{s}} \mathrm{O} \mathrm{O} \overline{\mathrm{ffe}} \overline{\mathrm{r}}$ & $\begin{array}{l}\text { Dummy } \\
\text { Variable }\end{array}$ & $\begin{array}{l}=1 \text { for households that got a retention offer from the call } \\
\text { center }\end{array}$ \\
\hline Fraction of Friends Get Offer & $\begin{array}{l}\text { Continuous } \\
\text { Variable }\end{array}$ & $\begin{array}{l}\text { Proportion of household's friends that got an offer from } \\
\text { the call center }\end{array}$ \\
\hline$\overline{\mathrm{O}} \overline{\mathrm{ffe}} \overline{\mathrm{A}} \mathrm{A}$ & $\begin{array}{l}\text { Dummy-- } \\
\text { Variable }\end{array}$ & $\begin{array}{l}=1 \text { for households that got a retention offer }{ }^{-} \text {from the } \\
\text { call center }\end{array}$ \\
\hline$\overline{\mathrm{O} f f} \overline{\mathrm{er}} \overline{\mathrm{B}}^{-}$ & $\begin{array}{l}\text { Dummy } \\
\text { Variable }\end{array}$ & $\begin{array}{l}=1 \text { for households that got a retention offer }{ }^{-} \bar{B} \text { from the } \\
\text { call center }\end{array}$ \\
\hline$\overline{\text { Offer }} \overline{\mathrm{C}}$ & $\begin{array}{l}\text { Dummy } \\
\text { Variable }\end{array}$ & $\begin{array}{l}=1 \text { for households that got a retention offer } \bar{C} \text { from the } \\
\text { call center }\end{array}$ \\
\hline
\end{tabular}

call center operator determines that the household is likely to churn then a deal is offered to try to retain her. A menu with three different levels of offers was available to call center operators for this purpose: i) offer A - reduced quality of service (such as fewer TV channels or lower Internet speed) for a lower price; ii) offer $\mathrm{B}$ - a discount of $\$ 3$ in the monthly bill corresponding to the free rental of the set-top-box; iii) offer $\mathrm{C}-\mathrm{a} \$ 7$ discount in the monthly bill. This menu of offers was the same for each and every call made during our experiment. Yet, call center operators were free to negotiate which offer to extend on a case by case basis but asked to try to retain the consumer at the least possible cost for the firm. In fact, call center operators were paid more if they were able to increase the households' contractual commitments and particularly high cash bonuses were paid when they were able to convince households to increase their monthly bill (which is extremely rare). The freedom given to call center operators to negotiate as best as possible with consumers introduces a second layer of selection in our experiment. The bottom-left and bottom-right panels in Figure 4 show that egos listed to be called by the firm were given a retention offer $34 \%$ of 
Table 3 Descriptive statistics for egos in the experiment per treatment condition.

\begin{tabular}{llrrr}
\hline \hline Treatment & Variable & Observations (Egos) & Avg. & Stdev \\
\hline Call No One & N. Alters & 3,996 & 1.890 & 1.067 \\
Call Alter & N. Alters & 3,895 & 1.890 & 1.080 \\
Call Ego & N. Alters & 33,312 & 1.830 & 1.045 \\
Call Ego and Alter & N. Alters & 3,820 & 1.849 & 1.018 \\
\hline Call No One & Churn Score & 3,996 & 0.689 & 0.274 \\
Call Alter & Churn Score & 3,895 & 0.689 & 0.271 \\
Call Ego & Churn Score & 33,312 & 0.715 & 0.276 \\
Call Ego and Alter & Churn Score & 3,820 & 0.691 & 0.273 \\
\hline Call No One & Monthly Bill & 3,996 & 55.722 & 13.951 \\
Call Alter & Monthly Bill & 3,895 & 55.824 & 13.846 \\
Call Ego & Monthly Bill & 33,312 & 56.300 & 13.593 \\
Call Ego and Alter & Monthly Bill & 3,820 & 55.637 & 14.001 \\
\hline Call No One & Lock-in Period & 3,996 & 30.801 & 77.817 \\
Call Alter & Lock-in Period & 3,895 & 33.937 & 87.800 \\
Call Ego & Lock-in Period & 33,312 & 30.814 & 79.083 \\
Call Ego and Alter & Lock-in Period & 3,820 & 30.863 & 80.765 \\
\hline
\end{tabular}

Note: monthly bill and lock-in period computed 30 days before treatment assignment.

Table 4 Descriptive statistics for alters in the experiment per treatment condition.

\begin{tabular}{llrrr}
\hline \hline Treatment & Variable & Observations (Alters) & Avg. & Stdedv \\
\hline Call No One & N. Alters & 6,545 & 7.677 & 32.444 \\
Call Alter & N. Alters & 6,235 & 6.150 & 9.633 \\
Call Ego & N. Alters & 55,925 & 6.272 & 9.093 \\
Call Ego and Alter & N. Alters & 6,043 & 5.923 & 5.561 \\
\hline Call No One & Churn Score & 6,545 & 0.230 & 0.268 \\
Call Alter & Churn Score & 6,235 & 0.222 & 0.262 \\
Call Ego & Churn Score & 55,925 & 0.240 & 0.275 \\
Call Ego and Alter & Churn Score & 6,043 & 0.224 & 0.261 \\
\hline Call No One & Monthly Bill & 6,545 & 54.420 & 23.603 \\
Call Alter & Monthly Bill & 6,235 & 54.846 & 23.513 \\
Call Ego & Monthly Bill & 55,925 & 54.761 & 24.040 \\
Call Ego and Alter & Monthly Bill & 6,043 & 54.704 & 23.624 \\
\hline Call No One & Lock-in Period & 6,545 & 128.649 & 232.783 \\
Call Alter & Lock-in Period & 6,235 & 131.182 & 234.552 \\
Call Ego & Lock-in Period & 55,925 & 132.666 & 235.551 \\
Call Ego and Alter & Lock-in Period & 6,043 & 132.708 & 234.636 \\
\hline
\end{tabular}

Note: monthly bill and lock-in period computed 30 days before treatment assignment.

Table 5 Descriptive statistics for clusters in the experiment per treatment condition.

\begin{tabular}{llrrr}
\hline \hline Treatment & Variable & Observations (N. Clusters) & Avg. & Stdev \\
\hline Call No One & N. Egos & 3,831 & 1.043 & 0.250 \\
Call Alter & N. Egos & 3,774 & 1.032 & 0.192 \\
Call Ego & N. Egos & 32,270 & 1.032 & 0.197 \\
Call Ego and Alter & N. Egos & 3,707 & 1.030 & 0.180 \\
\hline Call No One & N. Alters & 3,831 & 1.972 & 1.284 \\
Call Alter & N. Alters & 3,774 & 1.950 & 1.237 \\
Call Ego & N. Alters & 32,270 & 1.889 & 1.178 \\
Call Ego and Alter & N. Alters & 3,707 & 1.905 & 1.128 \\
\hline Call No One & Cluster Size & 3,831 & 3.015 & 1.433 \\
Call Alter & Cluster Size & 3,774 & 2.983 & 1.337 \\
Call Ego & Cluster Size & 32,270 & 2.921 & 1.277 \\
Call Ego and Alter & Cluster Size & 3,707 & 2.936 & 1.210 \\
\hline
\end{tabular}




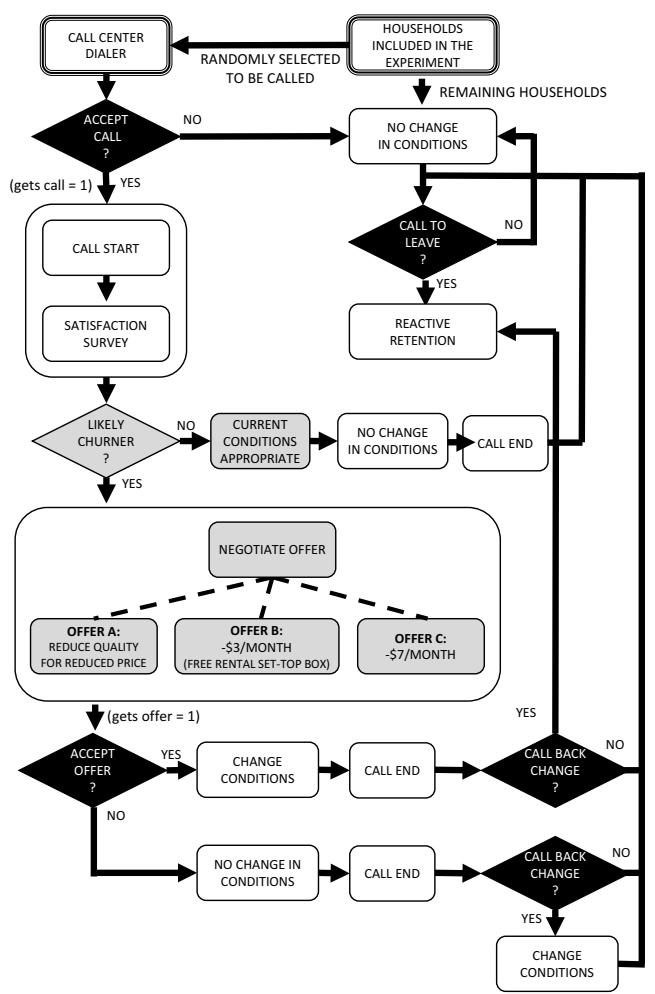

Figure 3 Decision stages and possible outcomes for households contacted by the firm during our experiment.

the time. Alters listed to be called by the firm received such offers $30 \%$ of the time. Obtaining an offer is conditional on getting a phone call from the call center. Therefore, roughly $2 / 3$ of the households that were reached during our experiment were given a retention offer. Appendix A provides additional descriptive statistics about which offers were accepted and not by households in the experiment. When an offer is made the household decides to accept or reject it on the spot. If the household accepts an offer from the call center operator then its conditions are updated and the call ends. However, the consumer has 15 days to call back and ask to revert to the previous conditions. If this is the case, then she enters reactive retention and the firm will try to retain her. If the household rejects the offer from the call center operator no change is made to her conditions and the call ends. However, a consumer that rejects such an offer also has 15 days to call back to accept it. All offers extended by TELCO under this type of campaign are registered in TELCO's information system and the company typically honors offers that it had previously extended to consumers. If this happens, the conditions of that household change. Finally, we note that whenever households are on the phone with a call center agent, they may ask for the service to be disconnected, in that case, consumers are routed towards the reactive retention team and the firms initiates all churn related procedures. 

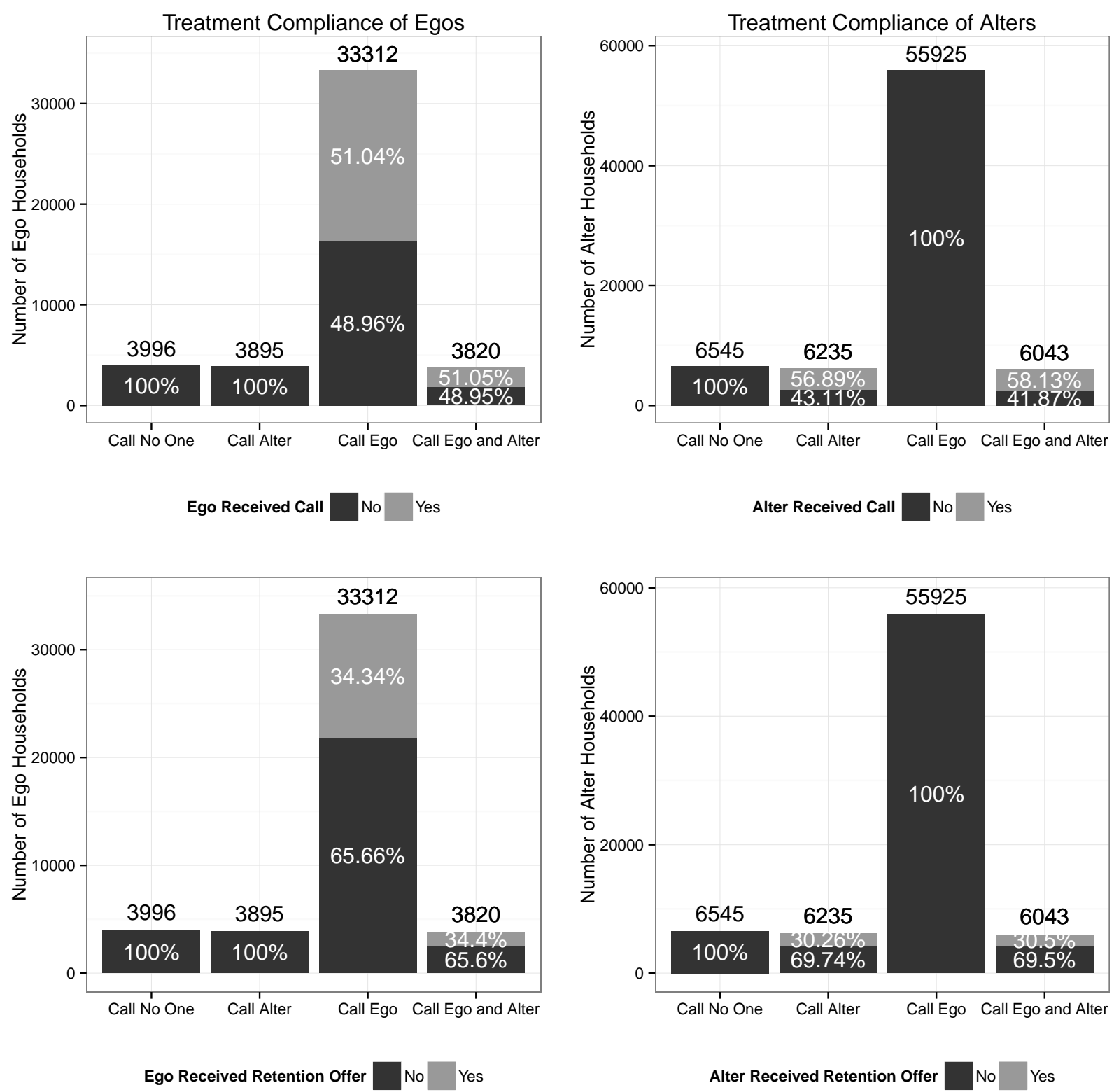

Figure 4 Compliance with treatment assignment during the experiment. The left panels show compliance across egos and the right panels show compliance across alters.

\section{Results}

In this section we measure the effect of proactive churn management in our setting by comparing the behavior of egos in the Call No One condition and in the Call Ego condition. We also measure the effect of socially-based proactive churn management by comparing the behavior of egos in the Call Ego condition and in the Call Ego and Alters condition. All these results measure the impact of our randomized assignment of households to treatment conditions before any selection occurs. This yields the effect of the Intention to Treat (ITT) households proactively across the population 
of likely churners at TELCO, which provides causal evidence of the effect of listing them to call. We also note that the probability of treatment assignment changed from month to month during our experiment. For example, the number of slots that were allocated to our experiment changed every month and in particular in June 2014. This means that the results that we present below are precision-weighted averages of monthly effects.

\subsection{Effect of Churn Management on Entering Retention and Churn}

We start by pooling all observations together to find whether there is some indication that our treatments yielded some effect. Figure 5 illustrates the results obtained for egos and alters in the top and bottom panels, respectively. The vertical axes on the left panels measure entry into reactive retention 120 days after treatment assignment. The vertical axes on the right panels measure churn in January 2016. The top panels show preliminary evidence that listing only alters to call does not change the likelihood at which egos enter reactive retention nor churn. However, listing egos to call reduces their likelihood of doing so and listing both egos and alters to call seems to yield even a larger effect. The bottom panels show that none of our treatments affected the likelihood at which alters enter reactive retention nor churn. Figure 6 illustrates what happens to monthly bills and to lock-in periods during our experiments. The top panels are for egos and the bottom panels are for alters. The vertical axes on the left panels measure changes in monthly bills. The vertical axes on the right panels measure changes in lock-in periods. The left top panel shows that listing only

alters to call does not change the monthly bills of egos. Listing egos to call reduces their monthly bills. Listing their alters to call reduces the monthly bills of egos by a similar amount, on average. The left bottom panel shows that listing egos to call does not change the monthly bill of alters. Listing alters to call reduces their monthly bills. The panels on the right show similar results for lock-in periods.

We estimate the magnitude of the causal effects of our treatments using the following model:

$$
Y_{j}=\zeta_{0}+\text { churn } \_ \text {score }_{j} \alpha_{1}+\text { treatment }_{\mathbf{j}} \alpha_{\mathbf{2}}+\theta_{t}+\epsilon_{j}
$$

where $j$ is a household in our experiment, $Y_{j}$ represents either entering reactive retention 120 days after treatment assignment or churn in January 2016, treatment $\mathbf{~}_{\mathbf{j}}$ is a vector of indicators for our treatment conditions and $\alpha_{2}$ is a vector of parameters indicating the corresponding levels of our dependent variable. Differences among these parameters will readily measure the effect of proactive churn management and of socially-based proactive churn management on our dependent variable. We use a Linear Probability Model (LPM) to estimate this equation. LPM is appropriate to identify the average causal effect of treatment assignment in randomized experiments with binary outcomes when the covariates of interest are sparse and discrete as in our case (Angrist 1991, Angrist et al. 
1996, Angrist 2001). For completeness, we present results using Probit in Appendix E, which are both qualitatively and quantitatively similar to the ones discussed below.

Table 6 shows the effect of treatment assignment on egos during our experiment. Columns (1) and (3) show that listing alters to call does not change the likelihood at which egos enter reactive retention nor churn. However, listing egos to call reduces the former by 1.7 percentage points, from a baseline of $20.7 \%$, and reduces the latter by 1.9 percentage points, from a baseline of $17.2 \%$. Columns (2) and (4) show that listing alters to call in addition to listing egos to call reduces the likelihood at which egos enter reactive retention by an additional 1.4 percentage points and the likelihood of churn by an additional 1.3 percentage points. Appendix G shows that these effects are not affected by tie strength nor by the degree centrality of the ego. Instead, in our setting, these effects are moderated by the proportion of alters that pick up the phone from the call center as shown in Appendix F. Finally, Table 7 shows results for the case of alters. All treatment conditions yield a similar likelihood of entering reactive retention and a similar churn rate.
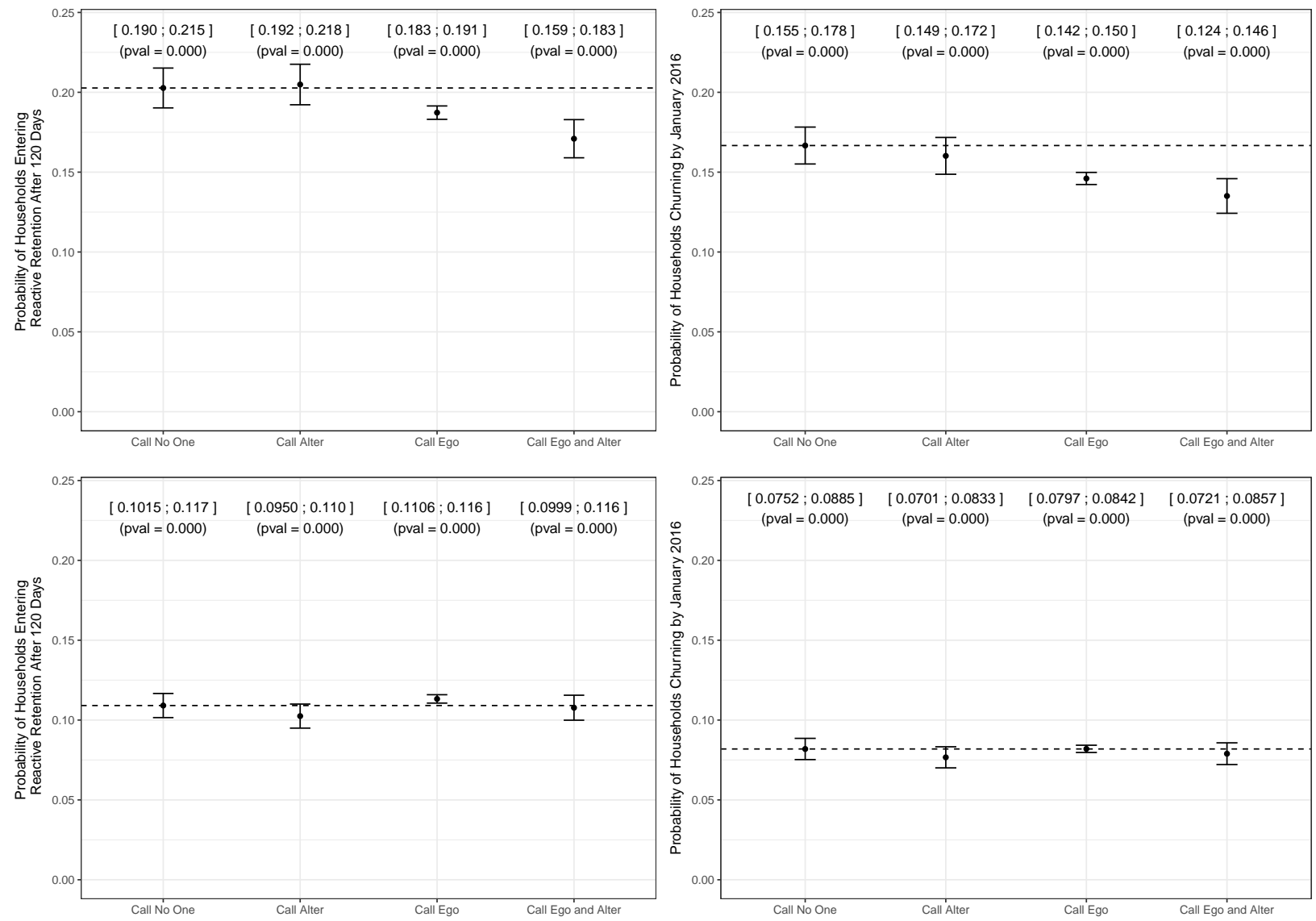

Figure 5 Likelihood of entering reactive retention during the mourning period (left panels) and likelihood of churn by January 2016 (right panels) per treatment condition. Top panels are for egos and the bottom panels are for alters. 

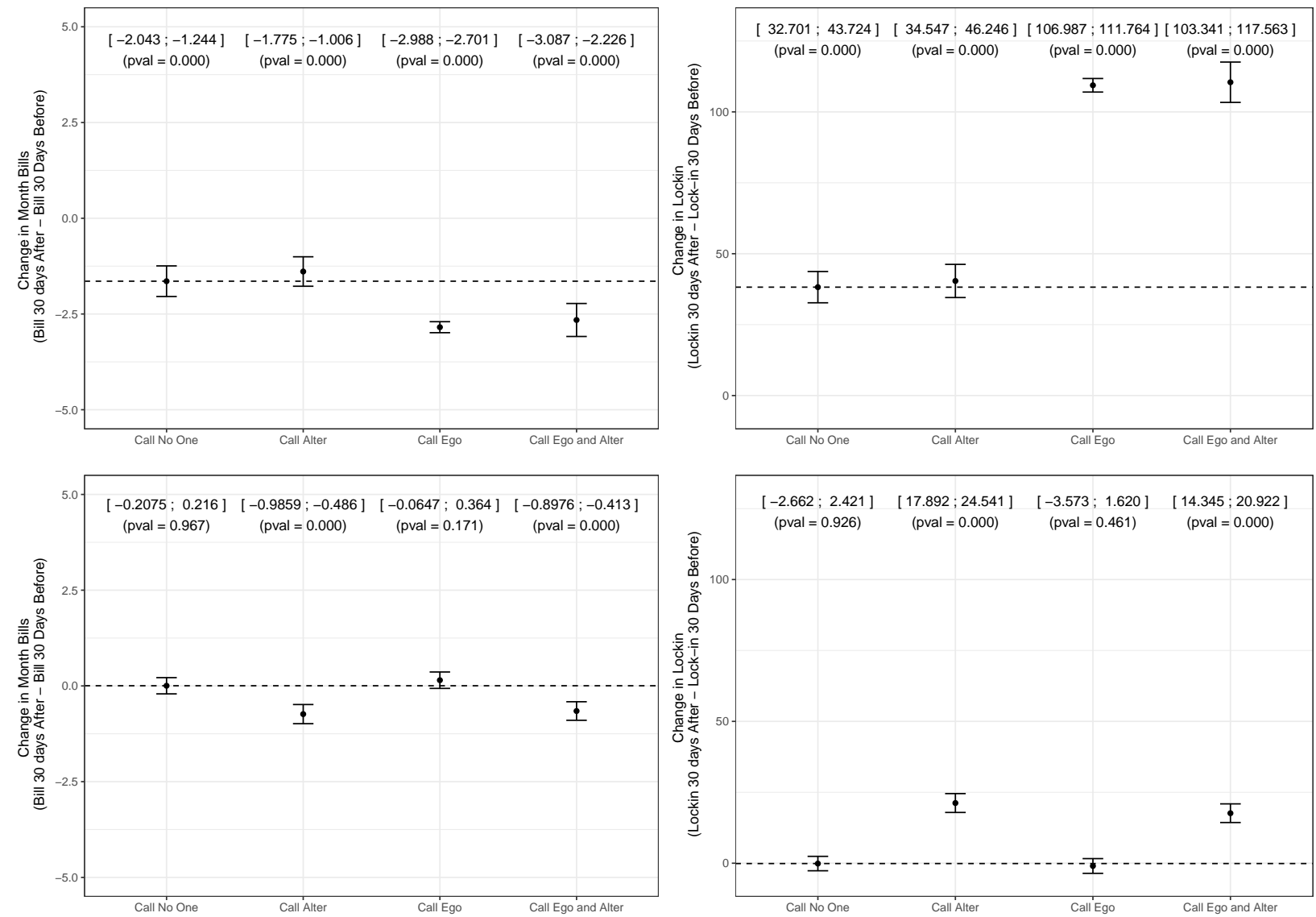

Figure 6 Change in the month bill (left panels) and lockin (right panels) of egos (top panels) and alters (bottom panels) over a period of 60 days: from 30 days before listing them to call to 30 days after listing them to call.

Our results show that calling only likely churners does not yield a spillover effect to alters and that calling only alters does not have spillover effect to egos. This is different from Ascarza et al. (2016a), where the authors find evidence of spillover effects. However, they study a product with explicit network externalities, which, as the authors argue, drives the results that they observe. Our case is different. We analyze a market without explicit network externalities and, in our setting, spillover effects arise only when both parties - the likely churner and her friends - are listed to be called by the firm. This shows that one must be very careful when generalizing spillover effects and that the effects identified in Ascarza et al. (2016a) may not generalize even within the telecommunications industry. More important, our findings show that in our setting a signal flows from alters to egos that lowers the likelihood at which the latter enter reactive retention and churn (in January 2016). This follows from the fact that with random assignment egos in the Call Ego and in the Call Ego and Alters conditions are, on average, similar on everything expect for the fact that their alters were also listed to be called by the firm under the latter condition. We cannot identify the channel used to transmit this signal nor we can be sure of what the signal is because 
Table 6 Effect of Intention To Treat on egos controlling for period dummies and churn score.

\begin{tabular}{|c|c|c|c|c|}
\hline & \multicolumn{4}{|c|}{ Dependent variable: } \\
\hline & \multirow{2}{*}{\multicolumn{2}{|c|}{$\begin{array}{c}\text { RR After } 120 \text { Days } \\
\text { LPM }\end{array}$}} & \multirow{2}{*}{\multicolumn{2}{|c|}{$\begin{array}{c}\text { Churn by Jan } 2016 \\
\text { LPM }\end{array}$}} \\
\hline & & & & \\
\hline & $(1)$ & $(2)$ & $(3)$ & $(4)$ \\
\hline Call No One & & $\begin{array}{l}0.017^{* *} \\
(0.007)\end{array}$ & & $\begin{array}{c}0.019^{* * *} \\
(0.006)\end{array}$ \\
\hline Call Alter & $\begin{array}{c}0.002 \\
(0.009)\end{array}$ & $\begin{array}{c}0.020^{* * *} \\
(0.007)\end{array}$ & $\begin{array}{l}-0.007 \\
(0.008)\end{array}$ & $\begin{array}{c}0.013^{* *} \\
(0.006)\end{array}$ \\
\hline Call Ego & $\begin{array}{c}-0.017^{* *} \\
(0.007)\end{array}$ & & $\begin{array}{c}-0.019^{* * *} \\
(0.006)\end{array}$ & \\
\hline Call Ego and Alter & $\begin{array}{c}-0.032^{* * *} \\
(0.009)\end{array}$ & $\begin{array}{c}-0.014^{* *} \\
(0.007)\end{array}$ & $\begin{array}{c}-0.032^{* * *} \\
(0.008)\end{array}$ & $\begin{array}{c}-0.013^{* *} \\
(0.006)\end{array}$ \\
\hline Churn Score & $\begin{array}{c}0.127^{* * *} \\
(0.035)\end{array}$ & $\begin{array}{c}0.127^{* * *} \\
(0.035)\end{array}$ & $\begin{array}{c}0.050 \\
(0.032)\end{array}$ & $\begin{array}{c}0.050 \\
(0.032)\end{array}$ \\
\hline Constant & $\begin{array}{c}0.103^{* * *} \\
(0.022)\end{array}$ & $\begin{array}{c}0.085^{* * *} \\
(0.022)\end{array}$ & $\begin{array}{c}0.172^{* * *} \\
(0.021)\end{array}$ & $\begin{array}{c}0.153^{* * *} \\
(0.020) \\
\end{array}$ \\
\hline Period Dummies & Yes & Yes & Yes & Yes \\
\hline Observations & 45,023 & 45,023 & 45,023 & 45,023 \\
\hline $\mathrm{R}^{2}$ & 0.002 & 0.002 & 0.003 & 0.003 \\
\hline Adjusted $\mathrm{R}^{2}$ & 0.002 & 0.002 & 0.003 & 0.003 \\
\hline Residual Std. Error & 0.391 & 0.391 & 0.355 & 0.355 \\
\hline F Statistic & $8.123^{* * *}$ & $8.123^{* * *}$ & $12.570^{* * *}$ & $12.570^{* * *}$ \\
\hline Note: & Cluster Ro & $\begin{array}{l}\text { nd Heterosk } \\
\text { rations clust }\end{array}$ & $\begin{array}{r}{ }^{*} \mathrm{p}<0.1 \\
\text { ic Consister } \\
\text { sy social ne } \\
\text { RR stan }\end{array}$ & $\begin{array}{l}5 ;{ }^{* * *} \mathrm{p}<0.01 \\
\mathrm{~d} \text { Errors in () } \\
\text { atment cluster } \\
\text { tive retention }\end{array}$ \\
\hline
\end{tabular}

we do not observe the messages exchanged between egos and alters. Yet, such a signal is certainly present in our setting and is responsible for the effects observed with socially-based proactive churn management.

\subsection{Mechanisms at Play in Socially-Based Proactive Churn Management}

Proactive churn management triggers a number of mechanisms that have been already discussed in the prior literature and that may arise in our setting. For example, egos go through a satisfaction survey when they pick up the call from the firm, which may reduce their likelihood of churn. As pointed out by Burez and Van den Poel (2007), satisfaction surveys may lead to positive evaluations that help retain consumers. However, this does not seem to be the case in our setting, as explained in detail in appendix $\mathrm{H}$. The egos that accept offers from the firm reduce their monthly bills (from $\$ 59.8 /$ month on average 30 days before being included in the experiment to $\$ 56.2 /$ month on average 30 days after being included in the experiment), which may, just per si, also lower their likelihood of churn. In addition, the offers that egos receive from the firm allow them to align their 
Table 7 Effect of the Intention To Treat on alters controlling for period dummies and churn score.

\begin{tabular}{lcc}
\hline \hline & & Dependent variable: \\
\cline { 2 - 3 } & RR After 120 Days & Churn by Jan 2016 \\
& $(1)$ & -0.004 \\
Call Alter & -0.006 & $(0.005)$ \\
Call Ego & $(0.005)$ & 0.001 \\
& 0.005 & $(0.004)$ \\
Call Ego and Alter & $(0.004)$ & -0.003 \\
& -0.001 & $(0.005)$ \\
Churn Score & $(0.006)$ & $0.093^{* * *}$ \\
& $0.113^{* * *}$ & $(0.005)$ \\
Flg. No Churn Score & $(0.005)$ & $0.048^{* * *}$ \\
& $0.038^{* * *}$ & $(0.003)$ \\
Constant & $(0.003)$ & $0.085^{* * *}$ \\
& $0.101^{* * *}$ & $(0.005)$ \\
\hline Period Dummies & $(0.005)$ & Yes \\
\hline Observations & Yes & 74,748 \\
$\mathrm{R}^{2}$ & 74,748 & 0.008 \\
Adjusted $\mathrm{R}^{2}$ & 0.007 & 0.008 \\
Residual Std. Error & 0.007 & 0.272 \\
F Statistic & 0.314 & $53.035^{* * *}$ \\
\hline \hline Note: & $44.269^{* * *}$ & ${ }^{*} \mathrm{p}<0.1 ;{ }^{* *} \mathrm{p}<0.05 ;{ }^{* * *} \mathrm{p}<0.01$ \\
& & RR stands for reactive retention \\
& & Observations clustered by social network treatment cluster \\
& & Cluster Robust and Heteroskedastic
\end{tabular}

monthly bills with those of their alters (the average monthly bill of alters is $\$ 55.3 /$ month 30 days before being included in the experiment), which is likely to increase their perception of fairness, thus potentially leading them to also churn less. For example, Bolton (1998) and Bolton and Lemon (1999) provide evidence that the perception of fairness reduces defection in environments where consumers can easily and meaningfully compare the prices that they are charged to those charged to their friends.

The mechanisms referred above can be at play in traditional proactive churn management and thus may explain the difference in the churn rates that we observe between egos in the Call No One and in the Call Ego conditions. The advantage of our study is that none of them are likely to play a role in explaining the lower churn rate of egos under socially-based proactive churn management. To see this, consider Table 8, which compares egos in the Call Ego and in the Call Ego and Alters conditions. Column (1) shows that egos in these two conditions pick up the phone from the firm at the same rate during our experiment. All egos that pick up the phone from the firm in these conditions go through the same satisfaction survey, that is, the survey included the same questions 
in the same order for each and every call made by any call center agent during our experiment. Column (2) in this table shows that egos in these two conditions not only pick up the phone from the firm at the same rate but that they also get offers from the firm at the same rate during the experiment. Columns (3)-(5) in this same table show that they even get the same offers equally often. Therefore, on average, egos in these two conditions have the same opportunities to lower their monthly bills if they accept the offers from the firm (note that even the monthly bill 30 days after treatment assignment for egos that accept offers and remain with the firm is similar, on average, for egos in these two treatment conditions: $\$ 56.2 /$ month under the Call Ego condition and $\$ 56.5 /$ month under the Call Ego and Alters condition). Furthermore, recall that, also on average, egos in these two conditions have the same monthly bills to begin with (as shown in table 3) and so do alters (as shown in table 4). Hence, reductions in the monthly bills of egos are very unlikely to explain the difference in their churn rates across these two conditions. Some alters under the Call Ego and Alters condition obtain and accept offers from the firm (which is unlikely under the Call Ego condition when alters are not listed to be called by the firm). Therefore, with respect to financial comparisons, if anything, the gaps in monthly bills between egos and alters reduce slightly less under socially-based proactive churn management, which would likely lead to a higher churn rate across egos under the Call Ego and Alters condition, which is not what we observe in our setting.

The discussion above provides evidence that other mechanisms must be at play in the case of socially-based proactive churn management. The local average treatment effects reported in table 12 in appendix $\mathrm{F}$ show that the effect of socially-based proactive churn management is associated to the alters that pick up the phone from the firm. However, in our setup, we are unable to split this effect into the one associated to the alters that get offers from the firm and the one associated to the alters that do not. Please refer to Appendix I for more details on why this is the case. It is possible that in our setting, part of the effect of listing alters to call on the churn rate of egos is associated to the alters that only get a satisfaction survey, which, according to the arguments above, may improve their opinion about the firm resulting in a more positive signal that they transmit to egos. This can trigger mechanisms associated to both conformity and financial comparisons. Cialdini and Goldstein (2004) define conformity as "the act of changing one's behavior to match the responses of others." If in our setting egos have a propensity to conform with their alters, the improved opinion that alters may have about the firm after going through the satisfaction survey may lead egos to stay more often with the firm under the Call Ego and Alters condition compared to what happens under the Call Ego condition (given that alters do not go through this survey under the latter condition). However, it may also be the fact that alters did not get offers from the firm that provides a different signal to egos under the Call Ego and Alters condition. Namely, egos 
have the same opportunities to align their monthly bills with those of their alters under the Call Ego condition and under the Call Ego and Alters condition. However, under the latter condition the alters that picked up the phone from the firm and did not get an offer may convey to egos that their monthly bills (that is, the monthly bills that egos can obtain if they accept the offers from the firm) seem hard to improve upon (because they were recently on the phone with the firm and were unable to get a better deal). This type of mechanism, which we may call "on-price" with low monthly bills for short, may also explain, in part, the observed lower churn rate across egos in our setting when alters are also listed to be called by the firm.

Finally, it is also possible that part of the effect that listing alters to call has on the churn rate of egos churn is associated to the alters that get offers from the firm after they go through the satisfaction survey. Getting such an offer might again improve their opinion about the firm, resulting in a more positive signal that they transmit to egos. Furthermore, the alters that accept these offers - and thus renew their contracts with the firm - have just made (recent conscious) decisions to stay, which egos may copy leading them to churn less under the Call Ego and Alters condition. In sum, both conformity and being "on-price" with low monthly bills may lead egos to churn less with socially-based proactive churn management.

Table 8 Comparing egos in the Call Ego and Call Ego and Alters conditions in terms of picking up the phone and obtaining offers from the TELCO.

\begin{tabular}{|c|c|c|c|c|c|}
\hline & \multicolumn{5}{|c|}{ Dependent variable: } \\
\hline & $\begin{array}{c}\text { Get Call } \\
(1)\end{array}$ & $\begin{array}{c}\text { Get Offer } \\
(2)\end{array}$ & $\begin{array}{c}\text { Offer A } \\
(3)\end{array}$ & $\begin{array}{c}\text { Offer B } \\
(4)\end{array}$ & $\begin{array}{c}\text { Offer C } \\
(5)\end{array}$ \\
\hline Call Ego and Alter & $\begin{array}{c}0.002 \\
(0.008)\end{array}$ & $\begin{array}{c}0.004 \\
(0.008)\end{array}$ & $\begin{array}{l}-0.002 \\
(0.002)\end{array}$ & $\begin{array}{l}-0.001 \\
(0.005)\end{array}$ & $\begin{array}{c}0.002 \\
(0.007)\end{array}$ \\
\hline Churn Score & $\begin{array}{c}-0.119^{* *} \\
(0.047)\end{array}$ & $\begin{array}{c}-0.132^{* * *} \\
(0.044)\end{array}$ & $\begin{array}{l}-0.009 \\
(0.011)\end{array}$ & $\begin{array}{c}-0.109^{* * *} \\
(0.030)\end{array}$ & $\begin{array}{l}-0.020 \\
(0.039)\end{array}$ \\
\hline Constant & $\begin{array}{c}0.565^{* * *} \\
(0.030) \\
\end{array}$ & $\begin{array}{c}0.411^{* * *} \\
(0.028) \\
\end{array}$ & $\begin{array}{c}0.040^{* * *} \\
(0.008) \\
\end{array}$ & $\begin{array}{c}0.170^{* * *} \\
(0.019) \\
\end{array}$ & $\begin{array}{c}0.207^{* * *} \\
(0.025) \\
\end{array}$ \\
\hline Period Dummies & Yes & Yes & Yes & Yes & Yes \\
\hline Observations & 37,132 & 37,132 & 37,132 & 37,132 & 37,132 \\
\hline $\mathrm{R}^{2}$ & 0.083 & 0.060 & 0.007 & 0.062 & 0.047 \\
\hline Adjusted $\mathrm{R}^{2}$ & 0.083 & 0.060 & 0.006 & 0.062 & 0.047 \\
\hline Residual Std. Error & 0.479 & 0.460 & 0.097 & 0.275 & 0.426 \\
\hline F Statistic & $372.823^{* * *}$ & $264.260^{* * *}$ & $27.357^{* * *}$ & $271.390^{* * *}$ & $204.269^{* * *}$ \\
\hline
\end{tabular}

Cluster Robust and Heteroskedastic Consistent Standar Errors in () Observations clustered by social network treatment cluster 


\subsection{The Effect of Churn Management on Firm Profit}

Blattberg et al. (2008) discusses reasons why proactive churn management may be very effective at keeping consumers but may fail to improve firm profits. In short, the firm tries to rescue likely churners offering them good deals. However, and along the process, the firm may also end up delighting non-likely churners, who obtain lower monthly bills though they would have not churned even if they were not given such good deals. The former improves firm profit while the latter does not. This trade-off is even trickier in the case of socially-based proactive churn management because in this case the firm also calls alters, who are unlikely to churn, and thus the likelihood of extending additional unnecessary deals may lower the profit of the firm even further. The appropriate way to determine the true effect of proactive churn management on the firm's profit is to measure its impact on Customer Lifetime Value (CLV). CLV is a function of the monthly bill, the survival with the firm and the discount rate. In our analysis, we set the monthly bill of each household in our experiment to its observed level 30 days after treatment assignment. We estimate survival as a function of treatment assignment using probabilistic continuous time models, which allow for churn rates to change over time (Fader and Hardie 2007, Schweidel et al. 2008). Appendix J provides the details of these computations and the results obtained. As expected, we find that the survival of egos increases when they are listed to be called by the firm and much more so when their alters are also listed to the called by the firm. The survival of alters does not change with treatment assignment. Finally, we set the discount rate to TELCO's Weighted Average Cost of Capital of $0.7 \% /$ month, as reported to us by company managers. We use these data to compute CLV over a 10-year horizon (the average TELCO household stays 62 months with the firm).

Table 9 shows the results obtained from regressing CLV on treatment assignment, which in our randomized setting allows for immediately identifying the effect of socially-based proactive churn management on the firm profit. Columns (1) and (2) show results for egos and columns (3) and (4) show results for alters. Column (1) shows that calling only alters does not change the CLV of egos in our setting. This column also shows that when only egos are listed to be called by the firm their CLV increases, on average, by $2.1 \%\left(51.1 /\left(2861-663^{*} 0.7\right)\right.$, where 0.7 is the average churn score across egos in our sample). This column also shows that when alters are listed to be called by the firm, in addition to egos, the CLV of egos increases, on average, by $6.4 \%(154.3 /(2861$ $\left.663^{*} 0.7\right)$ ). Column (2) shows that the difference between these statistics is statistically significant: $4.3 \%$ (given by $103.2 /(2911-663 * 0.7)$ ). This difference is the spillover effect associated to sociallybased proactive churn management, here measured in dollar terms for the firm. Columns (3) and (4) show that listing alters to be called by the firm, irrespective of whether egos are listed too, does not change their CLV, which is consistent with the fact that alters are unlikely to churn in our setting. Finally, and for robustness sake, columns (5) and (6) show the effects of our treatments 
over the clusters used in our experiment. The dependent variable in these regressions is the average CLV across egos and alters in each cluster (which adjusts for the fact that different clusters may have different sizes). The results in these columns confirm that in our setting listing likely churners to call is profitable for the firm and more so when their alters are also listed to be called. 


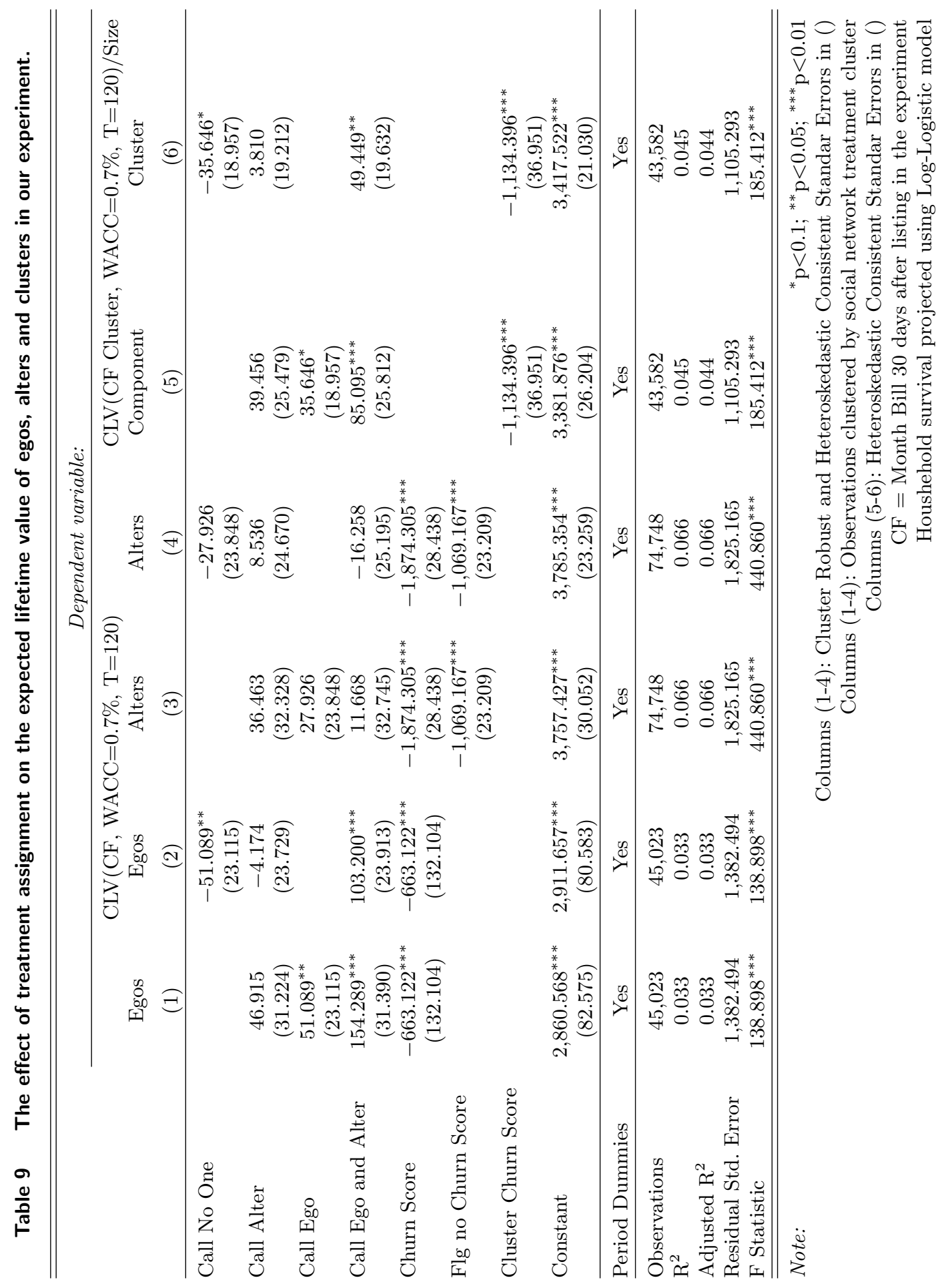




\section{Conclusions}

This paper studies whether a firm can actively use social network data to design an intervention that retains more consumers and increases firm profits than the current state of the art in proactive churn management. We do so in the context of triple play services, which is now becoming the standard mode of consumption in the telecommunications industry. In these markets, consumers become locked-in for several months before they can churn without paying financial penalties and there are no explicit network externalities in the way that they use services. Therefore, and at the outset, it would be unclear whether peer effects would play a role in this type of market and, if so, whether they would be economically significant to lead firms to consider them when retaining consumers. The main contribution of our work is to suggest a new type of intervention to help firms retain likely churners. In particular, we suggest that firms should also contact their friends. This strategy, which we call socially-based proactive churn management, is different from all previous approaches studied in the literature to perform proactive churn management, which involve contacting only likely churners. Two results from prior research on churn management in IT-related markets are inspirational for our research. First, proactive churn management may reduce churn. Second, one's decision to churn may be influenced by that of her friends. However, the latter was only observed before in markets with explicit network externalities. Yet, socially-based proactive churn management may work well if peer effects are strong enough. However, strong peer effects may not be sufficient for a firm to take advantage of this strategy because in the process of creating enough "goodwill" around the likely churner, the company might extend good deals to an unreasonable number of friends of likely churners (who could be unlikely to churn). This may hurt the firm's profit. If, however, a strong effect of peer influence can be achieved without losing too much revenue on the friends of the likely churners then it is possible that calling the latter becomes profitable for the firm.

We collaborated with a major telecommunications provider to design, implement and analyze outcomes of a randomized control trial at the household level to study the effect of socially-based proactive churn management. We started by using data from Call Detailed Records (CDRs) to draw a social graph across households and data from pay-TV subscriptions to develop a model to predict likely churners. Subsequently, and on a monthly basis during 8 consecutive months, we selected a random set of likely churners and their friends and we allocated them randomly across four different experimental conditions. In the Call No One condition all households were listed not to be called by the firm. In the Call Ego condition likely churners were listed to be called by the firm and their friends were listed not to be called by the firm. In the Call Alters condition the friends of likely churners were listed to be called by the firm and likely churners were listed not listed to be called by the firm. Finally, all households in the Call Ego and Alters condition were listed 
to be called by the firm. All calls were routed through the firm's call center. Randomly assigning households to these conditions allows us to immediately identify both the effect of proactive churn management - by comparing churn rates across the Call Ego and the Call No One conditions and the effect of socially-based proactive churn management - by comparing churn rates across the Call Ego and Alters and the Call Ego conditions. On average, we find that likely churners listed to be called whose friends were listed not to be called reduce their likelihood of churn by 1.9 percentage points $(\mathrm{p}$-value $<0.01$ ) from a baseline of $17.2 \%$. The likelihood of likely churners listed to be called whose friends were also listed to be called reduce the likelihood of churn by an additional 1.3 percentage points ( $\mathrm{p}$-value $<0.05$ ).

Our findings show that in our setting a signal flows from alters to egos that lowers the likelihood at which the latter enter reactive retention and churn. This signal may trigger several mechanisms that may lead egos to churn less with socially-based proactive churn management. The satisfaction survey that alters go through when they pick up the phone from the call center may, just per si, improve their opinion about the firm, which may lead them to transmit a more positive signal to egos. Likewise, for alters that obtain and accept an offer from the firm. If in our setting, egos have a propensity to conform with their alters, that is, if they exhibit a tendency to align their opinion with those of their alters and copy their behavior then these improved signals may lower the churn rate of egos when alters are also listed to be called by the firm compared to when they are not. However, such a lower churn rate across these egos may also because of the signal transmitted by the alters that pickup the phone from the firm and do not obtain offers. These alters may convey to their egos that if they accept the offers that they get from the firm then they obtain monthly bills that are hard to improve upon. This idea of "on-price" with low monthly bills may also decrease the likelihood of churn across egos whose alters had been listed to be called by the firm.

In addition, we use the framework proposed in Blattberg et al. (2008) to measure the effect of our interventions on firm profit using Customer Lifetime Value (CLV). The additional CLV associated to traditional proactive churn management is $\$ 51.1$ per likely churner, which represents an increase of $2.1 \%$ compared to reactive retention. These statistics are $\$ 154.3$ and $6.4 \%$, respectively, for the case of socially-based proactive churn management. Furthermore, we find no changes in the CLV of the friends of likely churners relative to reactive retention. Therefore, we show that in our context socially-based proactive churn management reduces churn among likely churners and increases firm profits. While a single empirical case is not enough to generalize results, we believe that our findings may extend to other contexts similar to ours, namely in subscription-based settings without explicit network externalities, where mechanisms akin to comparison and conformity play a role. Our paper suggests a new direction to enrich the firms' current portfolios of churn management strategies. Therefore, our results have significant implications for churn managers at firms that 
know, or that can proxy, the social network across their consumers and shows one way in which they can apportion value to these data.

Finally, we note that our work does not come without limitations. First, we do not observe the messages exchanged by egos and their friends. We have evidence that, in our setting, a signal flows from the latter to the former that reduces the churn rates of likely churners but we are unsure about what this signal is, when is it transmitted or what communication medium is used to transmit it. Second, part of the success of the churn management interventions that we test with our experiment is associated to the performance of the call center agents. This is not a special feature of our setting but rather an attribute common to all churn management exercises - in other words, one cannot expect to obtain good results from proactive churn management with unskilled call center agents. Third, we acknowledge that there are many ways in which one can identify customers at risk, in particular, the most likely churners might not be the most valuable customers to the firm nor the ones that are easier to retain. Therefore, the value generated by socially-based proactive churn management may even be higher that what we report in our paper if one combines targeting groups of consumers with more appropriate models to identify profitable targets.

\section{Acknowledgments}

Miguel Godinho de Matos acknowledges the support from the Portuguese Foundation for Science and Technology with grant reference UID/GES/00407/2013. We also thank our industry partner for their support. We thank the participants in the 13th ZEW Conference on The Economics of Information and Communication Technologies, the participants in the 2nd Conference on Digital Experimentation @ MIT and participants in the Heinz Research Seminar Series, in the Research Seminar Series at the Stern School of Management, NYU and in the Research Seminar Series at the Sloan School of Management, MIT for their comments and feedback to previous versions of this work. All remaining errors are our own.

\section{References}

Anderson, E. W., C. Fornell, D. R. Lehmann. 1994. Customer satisfaction, market share, and profitability: Findings from sweden. The Journal of Marketing 53-66.

Angrist, J. 1991. Instrumental variables estimation of average treatment effects in econometrics and epidemiology.

Angrist, J. D. 2001. Estimation of limited dependent variable models with dummy endogenous regressors. Journal of business \& economic statistics $\mathbf{1 9}(1)$.

Angrist, J. D., G. W. Imbens, D. B. Rubin. 1996. Identification of causal effects using instrumental variables. Journal of the American statistical Association 91(434) 444-455.

Aral, S., D. Walker. 2011. Creating social contagion through viral product design: A randomized trial of peer influence in networks. Management Science 57(9) 1623-1639. doi:10.1287/mnsc.1110.1421. 
Ascarza, E. 2017. Retention futility: Targeting high risk customers might be ineffective. Journal of Marketing Research 0(ja) null. doi:10.1509/jmr.16.0163. URL https://doi.org/10.1509/jmr.16.0163.

Ascarza, E., P. Ebbes, O. Netzer, M. Danielson. 2016a. Beyond the target customer: Social effects of crm campaigns. Journal of Marketing Research .

Ascarza, E., R. Iyengar, M. Schleicher. 2016b. The perils of proactive churn prevention using plan recommendations: Evidence from a field experiment. Journal of Marketing Research jmr-13.

Ascarza, E., S. Neslin, O. Netzer, Z. Anderson, P. Fader, S. Gupta, B. Hardie, A. Lemmens, B. Libai, D. Neal, et al. 2017. In pursuit of enhanced customer retention management. Working Paper .

Bapna, R., A. Umyarov. 2015. Do your online friends make you pay? a randomized field experiment in an online music social network. Management Science doi:Forthcoming.

Bensoussan, L., L. M. Del Bosch, F. Naud, P. Benichou. 2014. Churn value management: How can companies retain customers in an increasingly volatile world.

Berger, P. D., N. I. Nasr. 1998. Customer lifetime value: Marketing models and applications. Journal of interactive marketing 12(1) 17-30.

Blattberg, R. C., B.-D. Kim, S. A. Neslin, R. C. Blattberg, B.-D. Kim, S. A. Neslin. $2008 . \quad$ Database Marketing: Analyzing and Managing Customers. Springer.

Bloom, H. S. 1984. Accounting for no-shows in experimental evaluation designs. Evaluation review 8(2) $225-246$.

Bolton, R. N. 1998. A dynamic model of the duration of the customer's relationship with a continuous service provider: the role of satisfaction. Marketing science 17(1) 45-65.

Bolton, R. N., K. N. Lemon. 1999. A dynamic model of customers' usage of services: Usage as an antecedent and consequence of satisfaction. Journal of marketing research 171-186.

Burez, J., D. Van den Poel. 2007. Crm at a pay-tv company: Using analytical models to reduce customer attrition by targeted marketing for subscription services. Expert Systems with Applications 32(2) $277-288$.

Cialdini, R. B., N. J. Goldstein. 2004. Social influence: Compliance and conformity. Annu. Rev. Psychol. 55 $591-621$.

Eckles, D., B. Karrer, J. Ugander. 2014. Design and analysis of experiments in networks: Reducing bias from interference. arXiv preprint arXiv:1404.7530 .

Fader, P. S., B. G. Hardie. 2007. How to project customer retention. Journal of Interactive Marketing 21(1) $76-90$.

Friedman, J. H. 2001. Greedy function approximation: a gradient boosting machine. Annals of statistics $1189-1232$.

Green, H. 2016. Economic analysis of proposed sky/vodafone merger . 
Gupta, S., D. R. Lehmann, J. A. Stuart. 2004. Valuing customers. Journal of marketing research 41(1) $7-18$.

Jain, D., S. S. Singh. 2002. Customer lifetime value research in marketing: A review and future directions. Journal of interactive marketing 16(2) 34-46.

Kuhn, M. 2008. Building predictive models in r using the caret package. Journal of Statistical Software 28(5) $1-26$.

Lemmens, A., C. Croux. 2006. Bagging and boosting classification trees to predict churn. Journal of Marketing Research 43(2) 276-286.

Lemmens, A., S. Gupta. 2017. Managing churn to maximize profits .

Manski, C. F. 1993. Identification of endogenous social effects: The reflection problem. The review of economic studies $\mathbf{6 0}(3)$ 531-542.

McPherson, M., L. Smith-Lovin, J. M. Cook. 2001. Birds of a feather: Homophily in social networks. Annual review of sociology 415-444.

Muchnik, L., S. Aral, S. J. Taylor. 2013. Social influence bias: A randomized experiment. Science 341(6146) $647-651$.

Neslin, S. A., S. Gupta, W. Kamakura, J. Lu, C. H. Mason. 2006. Defection detection: Measuring and understanding the predictive accuracy of customer churn models. Journal of marketing research $\mathbf{4 3}(2)$ $204-211$.

Nitzan, I., B. Libai. 2011. Social effects on customer retention. Journal of Marketing 75(6) 24-38.

Peres, R., E. Muller, V. Mahajan. 2010. Innovation diffusion and new product growth models: A critical review and research directions. International Journal of Research in Marketing 27(2) 91-106.

Reichheld, F. 2003. The one number you need to grow. Harvard business review 81(12) 46-55.

Reichheld, F., W. Sasser. 1990. Zero defections: Quality comes to service. Harvard Business Review 68(5) $105-111$.

Reinartz, W., J. S. Thomas, V. Kumar. 2005. Balancing acquisition and retention resources to maximize customer profitability. Journal of Marketing 69(1) 63-79.

Reinartz, W. J., V. Kumar. 2003. The impact of customer relationship characteristics on profitable lifetime duration. Journal of marketing 67(1) 77-99.

Rosenbaum, P. R. 2007. Interference between units in randomized experiments. Journal of the American Statistical Association 102(477).

Rust, R. T., K. N. Lemon, V. A. Zeithaml. 2004. Return on marketing: Using customer equity to focus marketing strategy. Journal of marketing 68(1) 109-127.

Schweidel, D. A., P. S. Fader, E. T. Bradlow. 2008. Understanding service retention within and across cohorts using limited information. Journal of Marketing 72(1) 82-94. 
Shalizi, C., A. Thomas. 2011. Homophily and contagion are generically confounded in observational social network studies. Sociological Methods \& Research 40(2) 211.

Solomon, M. R. 2014. Consumer behavior: buying, having, and being. Prentice Hall Engelwood Cliffs, NJ.

Ugander, J., L. Backstrom, C. Marlow, J. Kleinberg. 2012. Structural diversity in social contagion. Proceedings of the National Academy of Sciences 109(16) 5962-5966.

Ugander, J., B. Karrer, L. Backstrom, J. Kleinberg. 2013. Graph cluster randomization: Network exposure to multiple universes $329-337$.

Venkatesan, R., V. Kumar. 2004. A customer lifetime value framework for customer selection and resource allocation strategy. Journal of marketing 68(4) 106-125.

Villanueva, J., S. Yoo, D. M. Hanssens. 2008. The impact of marketing-induced versus word-of-mouth customer acquisition on customer equity growth. Journal of marketing Research 45(1) 48-59.

Wasserman, S. 1994. Social network analysis: Methods and applications, vol. 8. Cambridge university press.

Winer, R. S. 2001. A framework for customer relationship management. California management review 43(4) 89-105.

Wooldridge, J. M. 2010. Econometric analysis of cross section and panel data. MIT press. 


\section{Appendix A: Retention Offers}

Table 10 provides descriptive statistics for the offers that ego households in the Call Ego and in the Call Ego and Alters conditions changed to upon being contacted by the firm. Offer C was the most accepted one during our experiment among the egos. Egos that accepted an offer from the firm churned 4 to 5 times less than egos that did not change their service because they either rolled back an offer they had previously accepted, did not accept the offer from the firm or did not get an offer from the firm. The majority of egos in our sample did not get an offer from the firm and a substantial number of egos that accepted an offer from the firm on the spot rolled back. A significant share of the latter churned.

Table 10 Descriptive statistics of outcomes for households in the Call Ego and in the Call Ego and Alters conditions during our experiment.

\begin{tabular}{lrcccc}
\hline \hline Households Action & $\mathrm{N}$ & $\begin{array}{c}\text { Avg. Month Bill } \\
\text { (30day aft.) }\end{array}$ & $\begin{array}{c}\text { Avg. Month Bill } \\
\text { (30day aft.) }\end{array}$ & $\begin{array}{c}\text { RR } \\
\text { (120day aft.) }\end{array}$ & $\begin{array}{c}\text { Churn } \\
\text { (Jan 2016) }\end{array}$ \\
\hline Changed to Offer A & 132 & 60.395 & 54.934 & 0.129 & 0.038 \\
Changed to Offer B & 1,290 & 62.474 & 59.774 & 0.083 & 0.030 \\
Changed to Offer C & 4,672 & 59.024 & 51.713 & 0.085 & 0.053 \\
No change (initially accepted an offer) & 1,487 & 53.279 & $50.012^{+}$ & 0.176 & 0.165 \\
No change (did not accept an offer) & 5,171 & 55.254 & $54.152^{+}$ & 0.240 & 0.174 \\
No change (did not get an offer) & 24,380 & 55.732 & $53.436^{+}$ & 0.200 & 0.162 \\
\hline
\end{tabular}

+ Change in average monthly bills is due to households that churn and whose monthly bill is set to zero.

\section{Appendix B: Churn Prediction Model}

We used the pre-experimental dataset for the period December 2013 - March 2014 to build a classification algorithm that allows for predicting in advance households that are likely to churn. We used the Caret framework to train and evaluate the performance of different machine learning algorithms (Kuhn 2008) on their ability to predict churn. All algorithms were trained and tested using 10 fold cross validation. A detailed description of each algorithm is beyond the scope of this paper. Refer to Kuhn (2008) for a complete overview. Model acronyms in our figures are provided exactly as by Kuhn (2008). Engineering all the features that were included as candidate predictors of churn in our model was done in partnership with the churn management team at TELCO. We include a large number of features available to us in these models and we deliberately decided to use machine learning algorithms that include automated feature selection over other algorithms in which feature selection is a separate modeling step. Figure 7 shows that most models fit our data well. In particular, the area under the curve (AUC/ROC) - giving us the probability that a classifier ranks a randomly chosen positive instance higher than a randomly chosen negative instance - is near $80 \%$ across all algorithms used. This threshold is usually employed as rule of thumb criterium in predictive analytics to determine whether a model is sufficiently good.

In our setting Gradient Boosted Model trees (GBM) (Friedman 2001) achieved better scores in (almost) every performance metric. Thus, we opted for GBM in our study. Figure 8 shows several learning plots for our predictive model exercise. These plots show that in our setting slow learners with moderate tree complexity performed better. Figure 9 plots the ROC curve of our GBM configured to the parameters that 


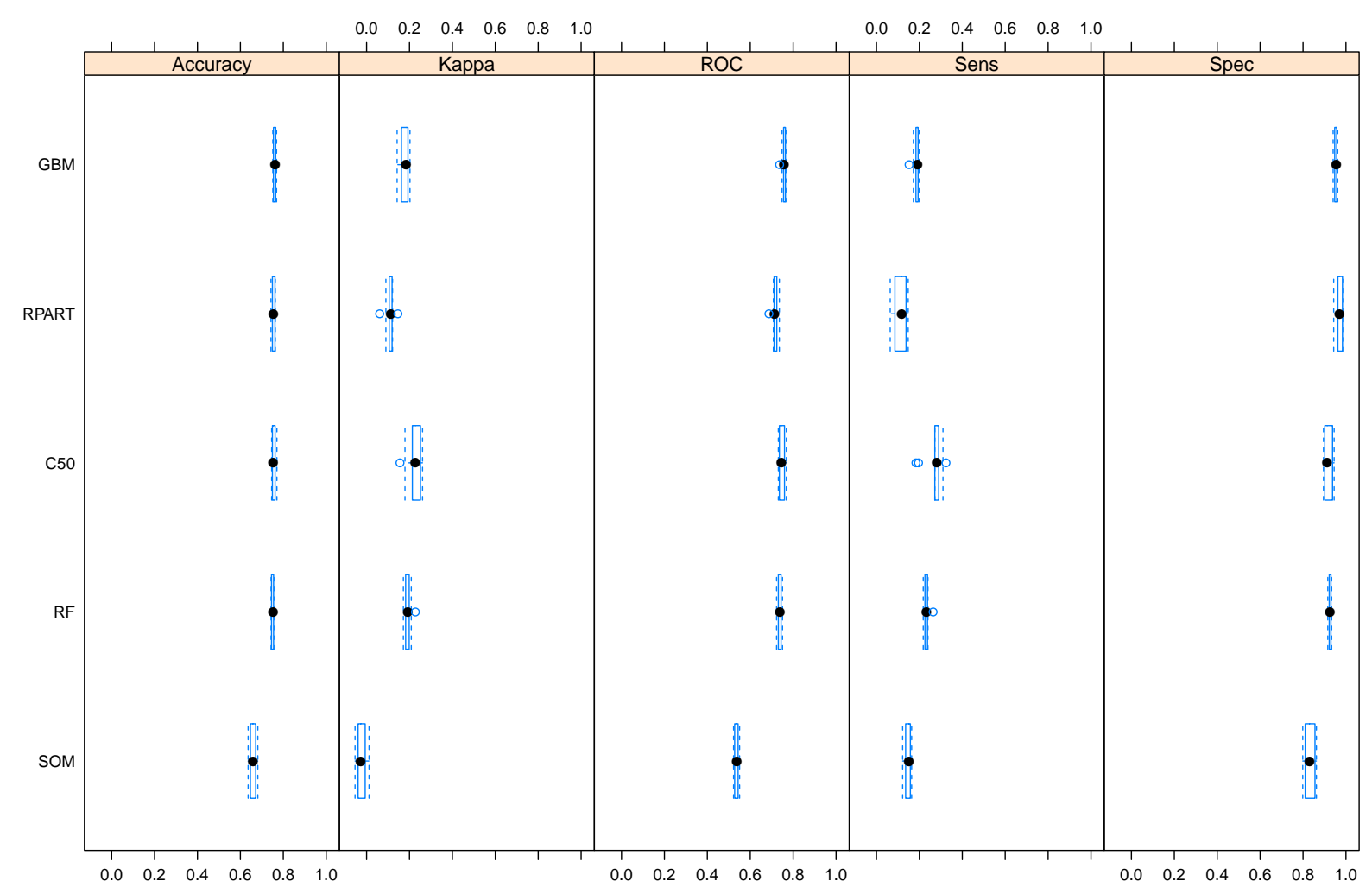

Figure 7 Performance of the machine learning algorithms tested.

optimize performance. Figure 10 shows the corresponding lift. These figures show that our model provided significant lift (compared to random classification) at small thresholds that face quickly when classifying positive instance became less strict (i.e. positive instances classified with smaller churn scores). Over time, we selected a different threshold to build our classifier from the GBM algorithm. We did so because (1) we retrained our model twice during our experiment, implying that each time the model was estimated the churn scores were calibrated differently, and (2) we built our classifier to roughly match the capacity available in TELCO's call center. Finally, and for completeness, Figure 11 provides a list of the top 10 features that predict churn. Variable importance is standardized to the contribution of most relevant feature and tracks the reduction in the generalized cross-validation statistic as covariates are added. Household tenure, contractual obligations, monthly bill, the age of the contract holder and the amount off-price are among the characteristics that better discriminate likely churners.

\section{Appendix C: Sample Size Calculation to Size our Experiment}

We define the sample size for this experiment in a way that allow us to identify an effect that renders proactive churn management profitable on the egos. Finding an effect that would not generate profit would be uninteresting from an economic point of view. Our proactive churn management campaign is profitable on the egos as long as the benefits from each retained household supersede the costs of calling households that 


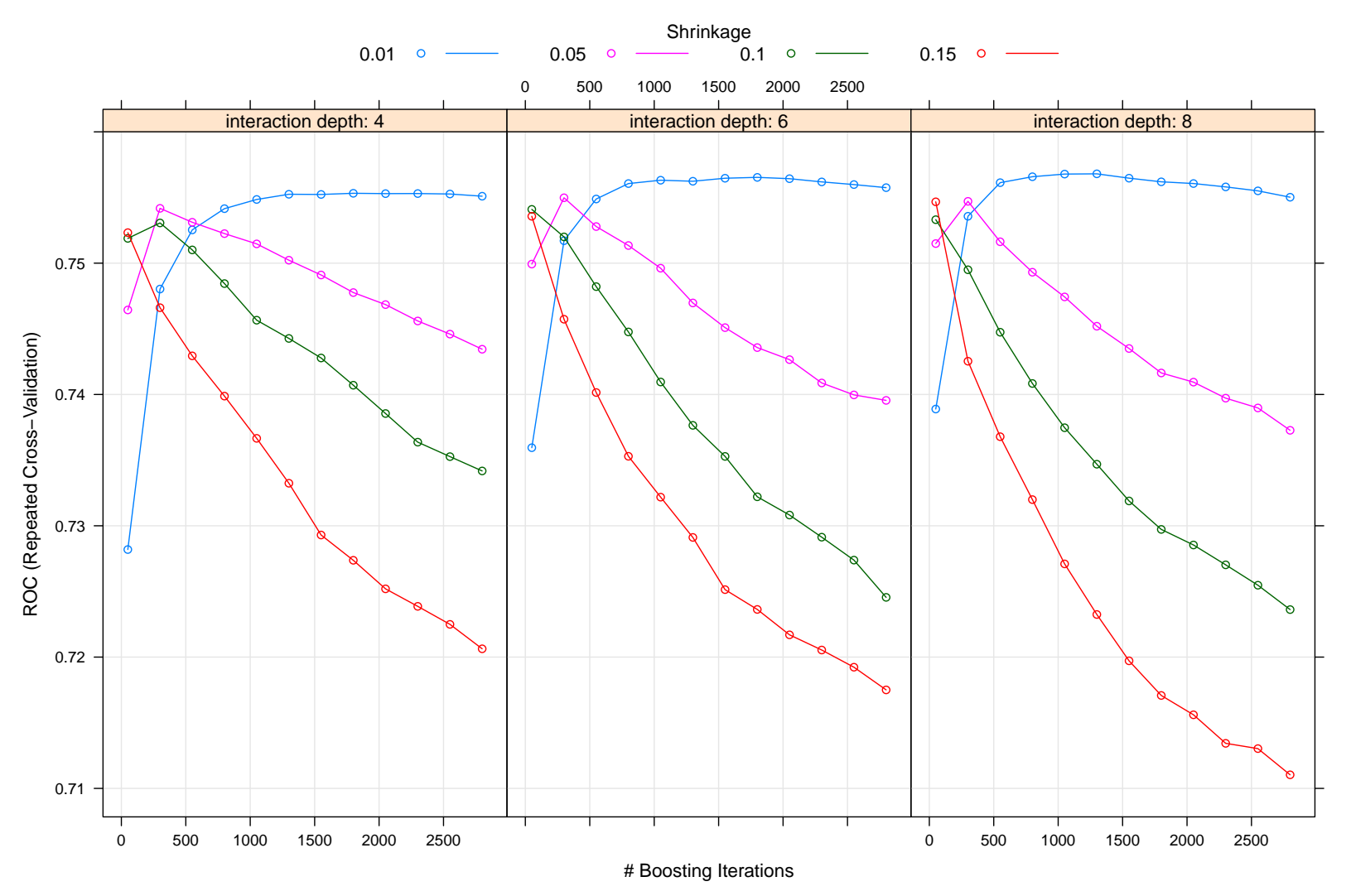

Figure 8 Learning plots for the parameterization of the GBM algorithm.

are not retained and that would not churn (false positives). We determine the minimum effectiveness of this operation to break-even using the following data obtained from churn managers at TELCO: each recovered household yields an NPV of $\$ 2589$ ( $\$ 45 /$ month over a lifetime of 6 years using a discount rate of $9 \% /$ year minus $\$ 4$ for the cost of the call); the expected net loss from calling a false positive is $\$ 219$ ( $\$ 4$ for the cost of the call plus $\$ 5 /$ month over a lifetime of 11 years using a discount rate of $9 \% /$ year and assuming that about $50 \%$ of the contacted households will accept retention offers). The operation would be profitable if, for each recovered household (true positive), at most $11.8(\$ 2589 / \$ 219)$ households were contacted. In other words, the operation would be profitable if the ratio between recovered households and contacted households is at least $0.085(\$ 219 / \$ 2589)$. Thus, the minimum effectiveness of the call center for this operation to be profitable is $8.5 \%$.

An important restriction to the design of this experiment was that the call center capacity was set close to 5,000 calls/month at the beginning of the experiment. An additional restriction imposed by TELCO was that the majority of the call center effort should be allocated to contact likely churners. We established that each month at most $25 \%$ of the households identified as likely churners would be held out. Moreover, some call center capacity would be needed to contact alters. Given these restrictions, we used 3,000 call center slots per month to contact egos and we listed 1,000 egos per month not to contact. The remaining call center capacity of about 2,000 sots per month was used to contact alters. Given that on average 19\% of TELCO's households enter retention every 120 days and that the number of egos listed to be contacted is expected to 


\section{ROC curve}

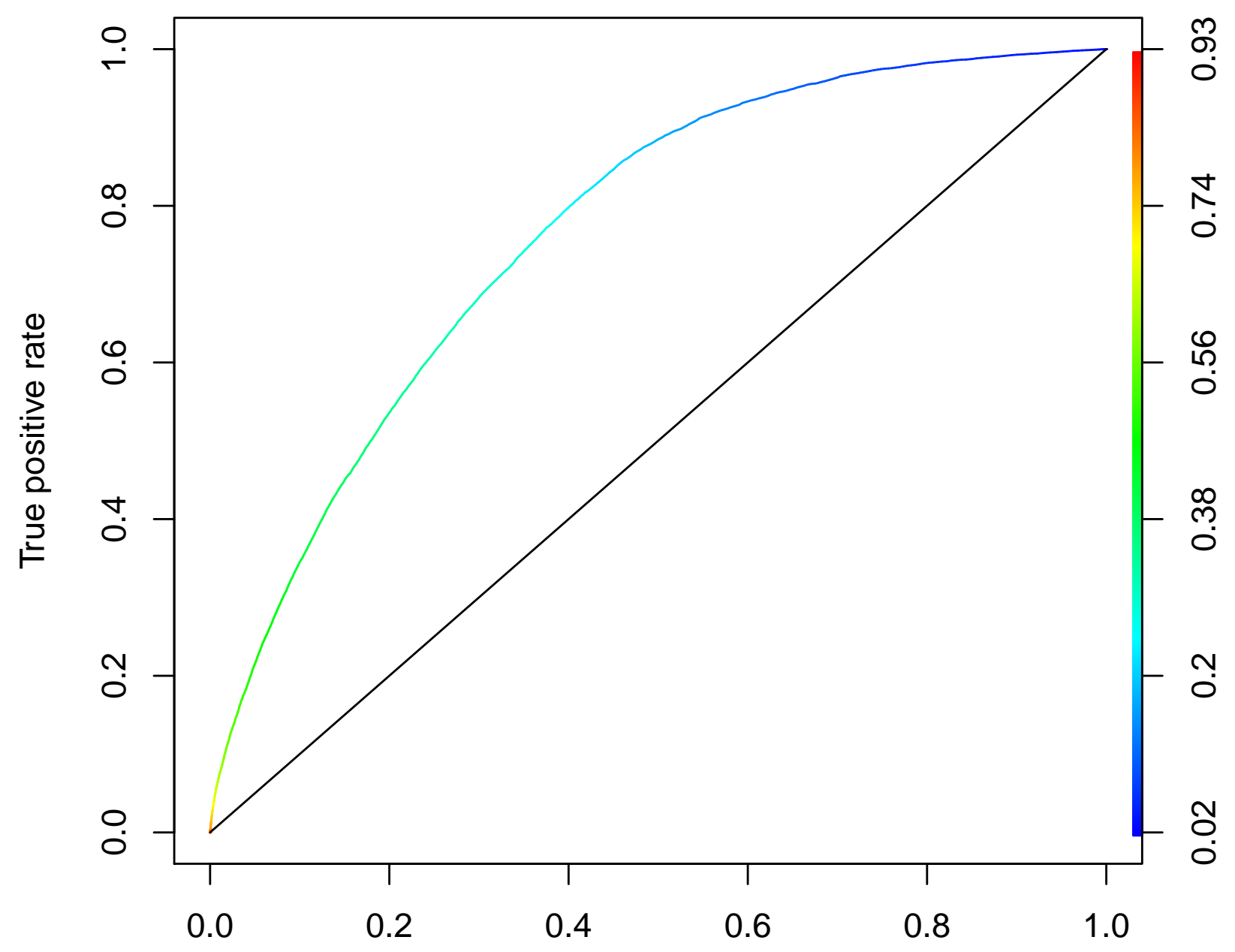

False positive rate

Figure 9 ROC space of the fitted BGM trees algorithm.

be roughly 3 times those listed not to be contacted, the minimum number of egos listed not to be contacted required to identify an effectiveness of $8.5 \%$, with $95 \%$ confidence and $80 \%$ power, is 5,976 . Given that we list 1,000 ego households not to be contacted per month our experiment should last at least 6 months, to which we added an extra 2 months to account for potential loss of data.

Finally, we note that a limitation of our computations for minimum sample size is that they focus only on finding an effect on egos and did not take into account what could happen to alters. We ended up splitting the 2,000 call center slots used to contact alters evenly between alters of egos listed to contact and alters of egos listed not to contact. Therefore, each month about 500 egos listed to be contacted had their alters listed to be contacted and about 500 egos listed not to be contacted had their alters listed to be contacted. 


\section{Lift curve}

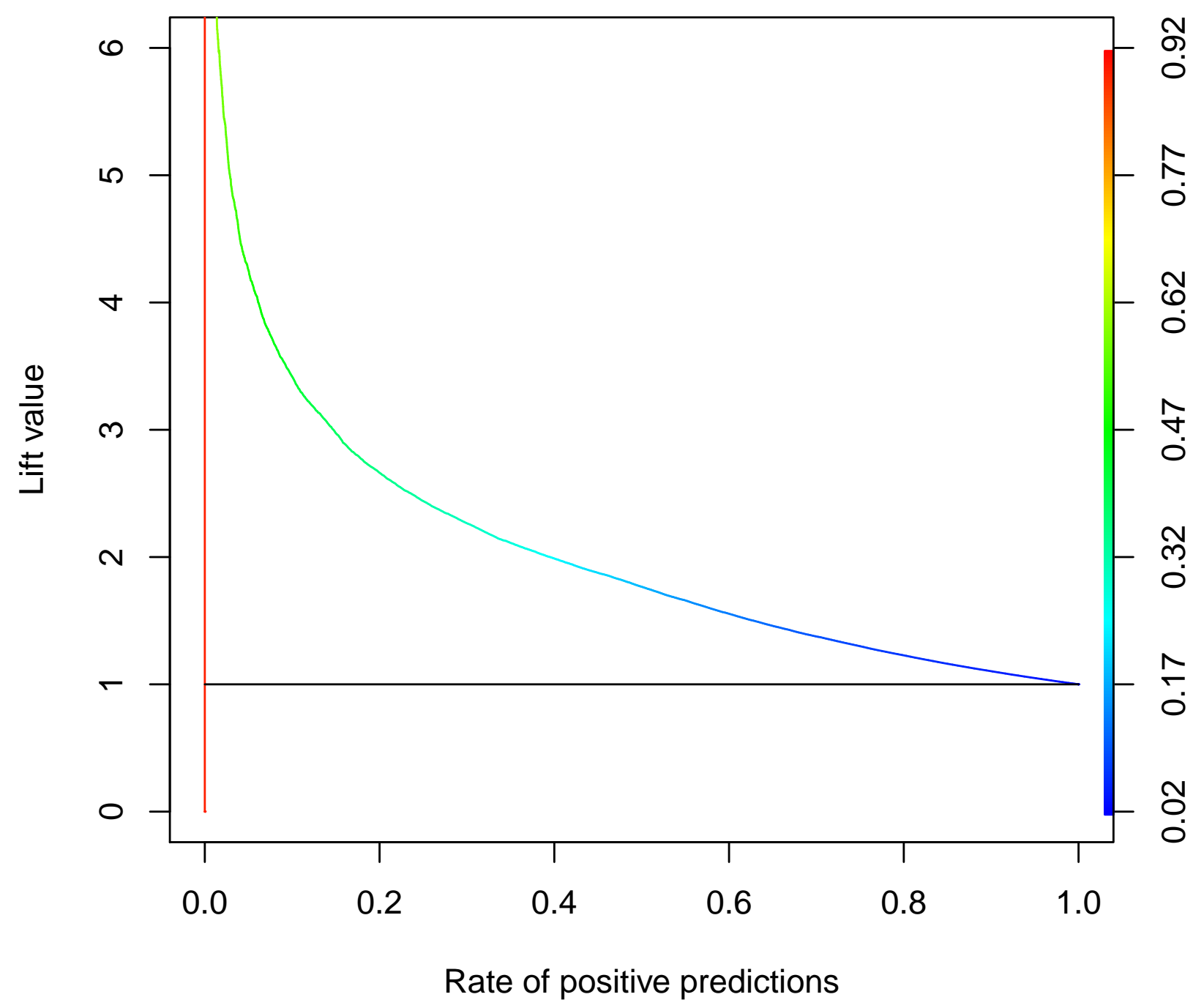

Figure 10 Lift curve of the fitted GBM trees algorithm.

\section{Appendix D: Descriptive Statistics of Pre-treatment Covariates}

The top left panel in Figure 12 shows that, on average, the churn score obtained from our churn prediction model was similar for egos and alters across all treatment conditions at any point in time during our experiment. Variations in the average churn score across time periods are explained by seasonality and by the fact that we retrained the predictive churn model when TELCO provided us with new data during the experiment. The remaining panels in this figure repeat this analysis for number of friends, monthly bills and lock-in periods (number of days to contract expiry). Likewise, and on average, egos in all treatment conditions are statistically similar with respect to these covariates as highlighted by the F-test for the analysis of variance run across the four experimental conditions at each point in time and for each covariate. Likewise, 


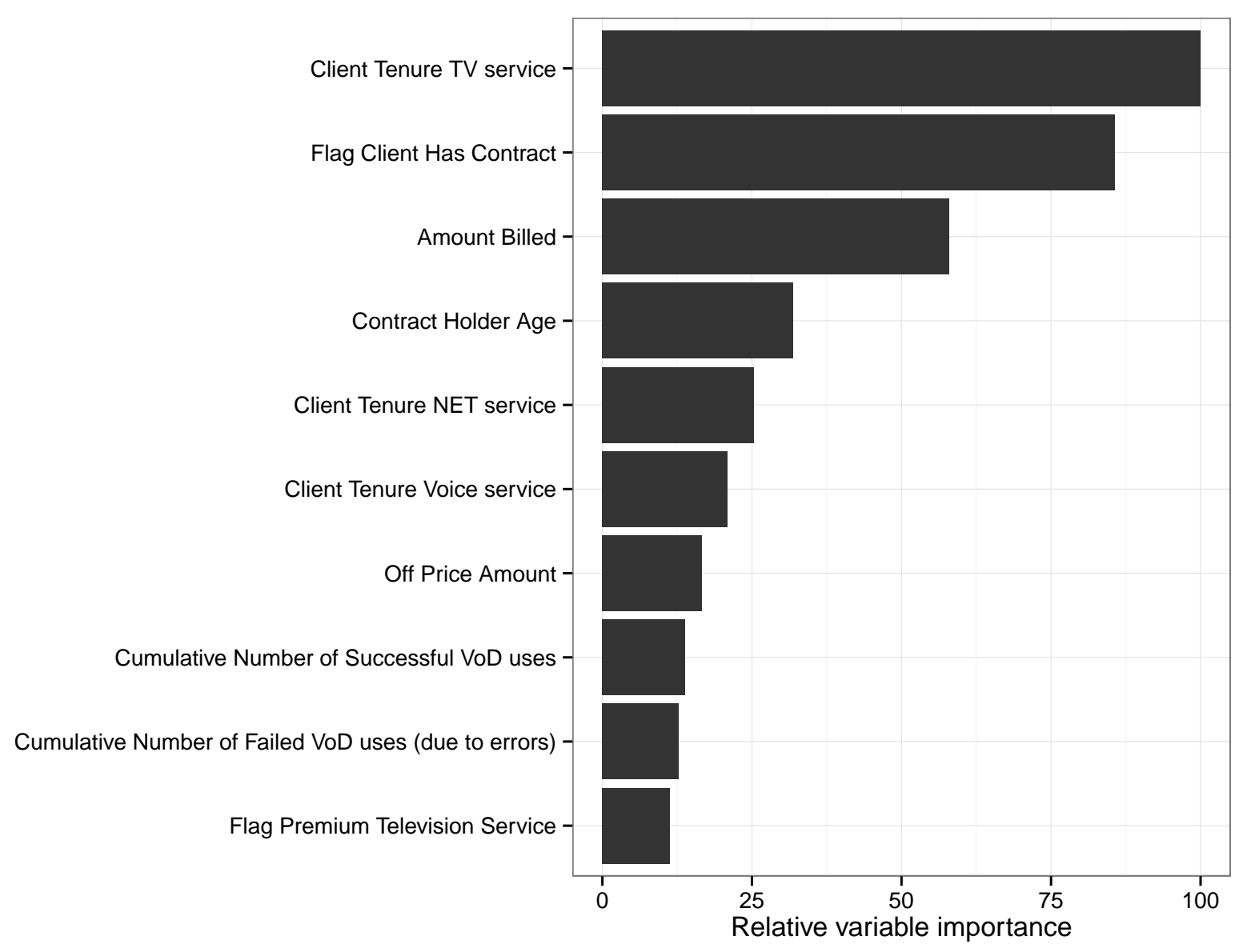

Figure 11 Variable importance plot for the GBM trees algorithm.

for alters. Figure 13 shows that, on average, the likelihood of churn was the same for alters across the four different treatment conditions at any point in time during our experiment. 

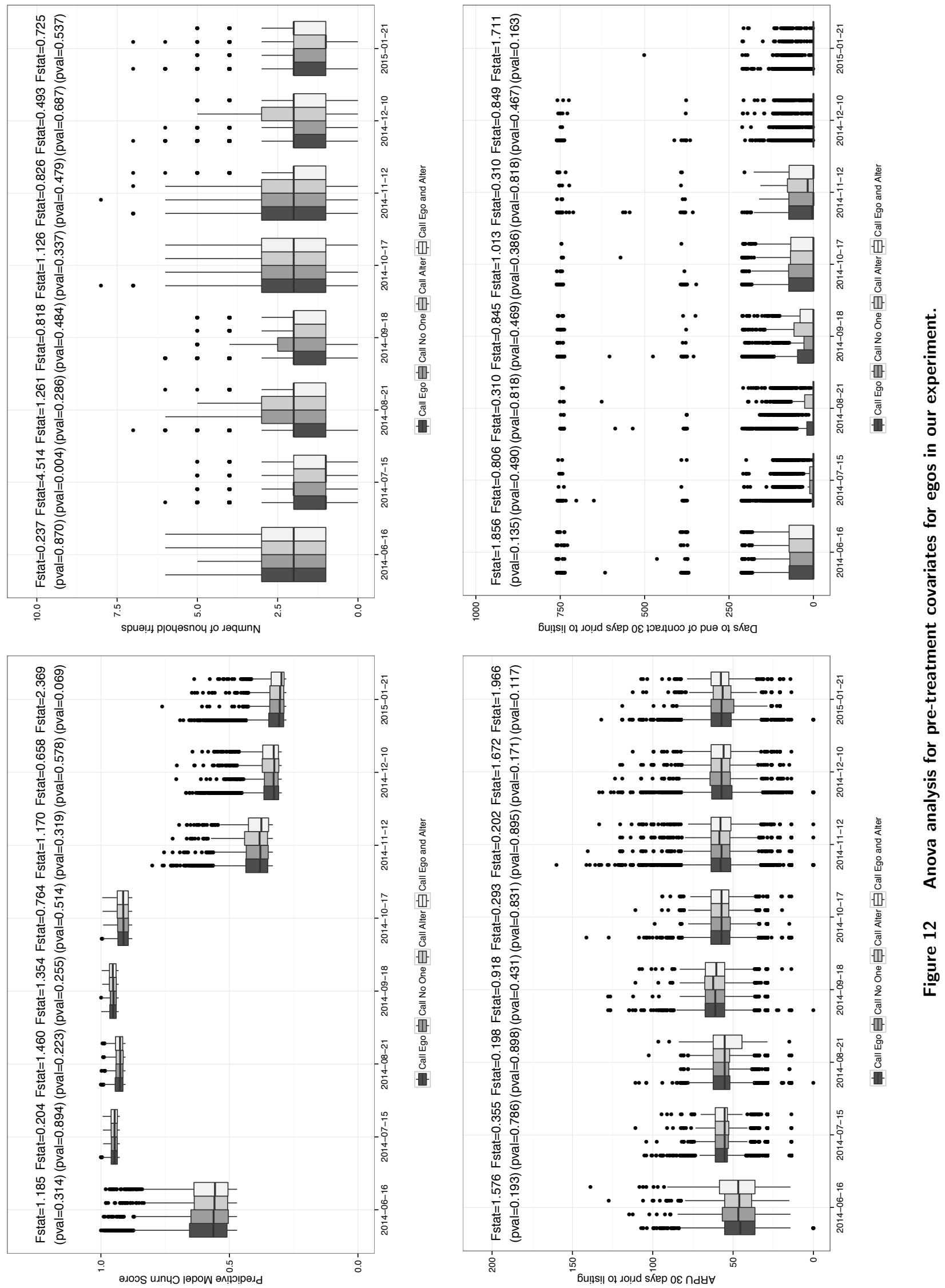
41
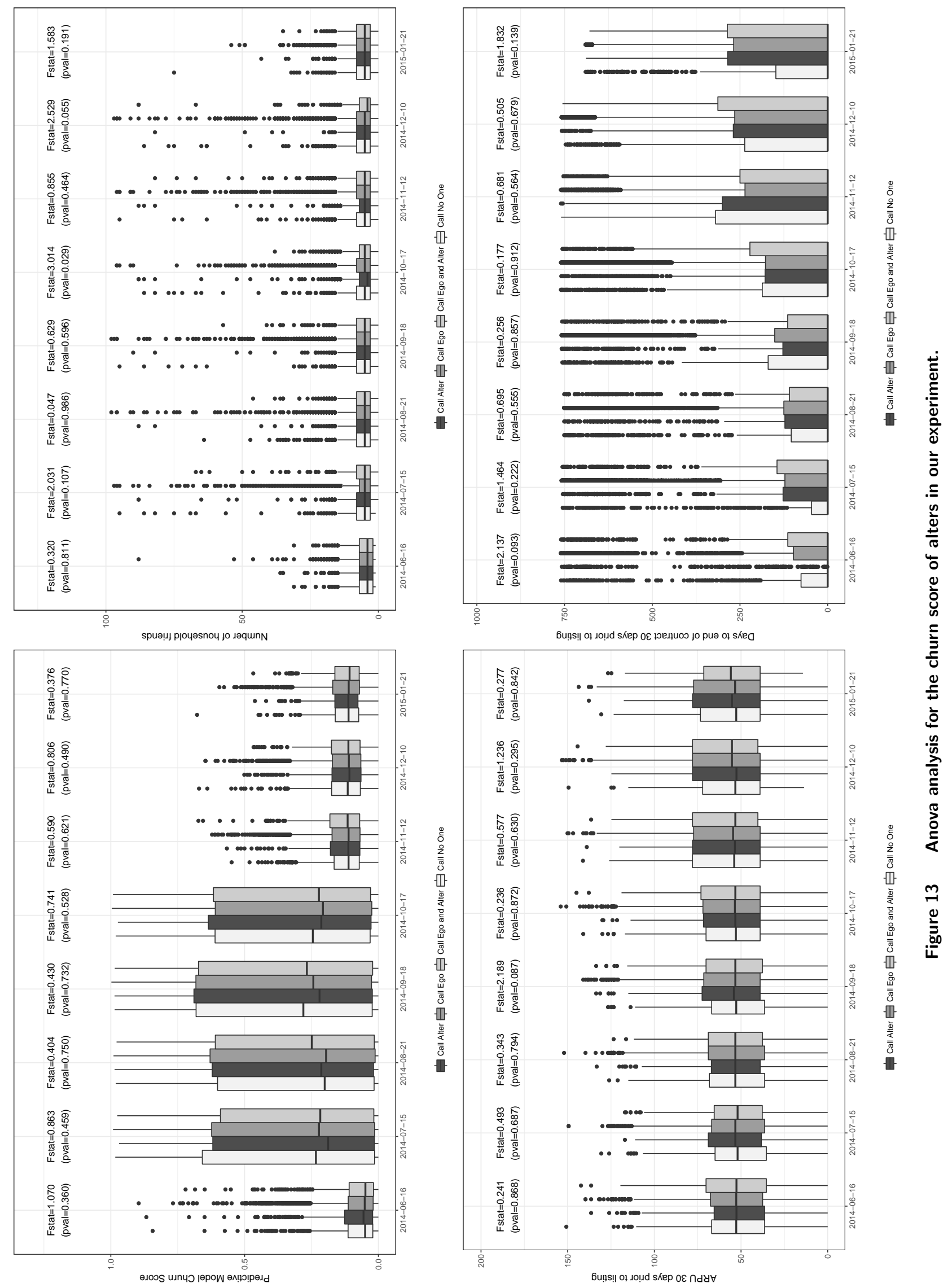


\section{Appendix E: Probit Functional Form}

Table 11 shows the effect of our treatments on the likelihood of entering retention 120 days after treatment assignment and on churn in January 2016 for egos in our experiment using a Probit functional form. These results are in line, both qualitatively and quantitatively, with the ones presented in the main body of the paper using a Linear Probability Model.

Table 11 Treatment effects on the likelihood of entering reactive retention and churning across egos.

\begin{tabular}{lcccc}
\hline \hline & \multicolumn{3}{c}{ Dependent variable: } \\
\cline { 2 - 5 } & \multicolumn{2}{c}{ RR After 120 Days } & \multicolumn{2}{c}{ Churn by Jan 2016} \\
& $(1)$ & $(2)$ & $(3)$ & $(4)$ \\
& \multicolumn{3}{c}{$0.064^{* * *}$} \\
Call No One & $(0.024)$ & $0.080^{* * *}$ \\
& & & $(0.025)$ \\
Call Alter & 0.008 & $0.071^{* * *}$ & -0.027 & $0.053^{* *}$ \\
& $(0.032)$ & $(0.024)$ & $(0.033)$ & $(0.026)$ \\
Call Ego & $-0.064^{* * *}$ & & $-0.080^{* * *}$ & \\
& $(0.024)$ & & $(0.025)$ & $-0.058^{* *}$ \\
Call Ego and Alter & $-0.118^{* * *}$ & $-0.054^{* *}$ & $-0.138^{* * *}$ & $(0.027)$ \\
& $(0.033)$ & $(0.026)$ & $(0.035)$ & 0.210 \\
Churn Score & $0.472^{* * *}$ & $0.472^{* * *}$ & 0.210 & $(0.130)$ \\
Constant & $(0.125)$ & $(0.125)$ & $(0.130)$ & $-1.030^{* * *}$ \\
& $-1.207^{* * *}$ & $-1.271^{* * *}$ & $-0.949^{* * *}$ & $(0.082)$ \\
\hline Period Dummies & $(0.082)$ & $(0.080)$ & $(0.085)$ & Yes \\
\hline Observations & Yes & Yes & Yes & 45,023 \\
Log Likelihood & 45,023 & 45,023 & 45,023 & $-18,820.000$ \\
Akaike Inf. Crit. & $-21,766.000$ & $-21,766.000$ & $-18,820.000$ & $37,664.000$ \\
\hline \hline
\end{tabular}

Note:

${ }^{*} \mathrm{p}<0.1 ;{ }^{* *} \mathrm{p}<0.05 ;{ }^{* * *} \mathrm{p}<0.01$

Cluster Robust and Heteroskedastic Consistent Standar Errors in ()

Observations clustered by social network treatment cluster

$\mathrm{RR}$ stands for reactive retention 


\section{Appendix F: Local Average Treatment Effects for Egos}

We follow Angrist et al. (1996) and report Local Average Treatment Effects (LATEs) using treatment assignment as an instrumental variable for treatment compliance. We note that all panels in Figure 4 show perfect compliance with the hold out conditions. Therefore, all egos listed not to be called by the firm were indeed not called. Therefore, we have one-side non-compliance with treatment assignment (Bloom 1984) and thus our LATE estimates reported below measure the Average Effect on the Treated (ATT) (Angrist et al. 1996). These results are consistent with the results presented in the main body of the paper and show that egos that pick up the phone from the call center more often and egos with more alters that pick up the phone from the call center are less likely to enter reactive retention and churn. Columns (1) and (3) in this table include egos in the Call No One condition and in the Call Ego condition and use whether the ego was listed to be called by the firm as an instrument for whether she picked up the call from the firm. Columns (2) and (4) in this table include egos in the Call Ego condition and in the Call Ego and Alters condition and use whether alters were listed to be called by the firm as an instrument for the proportion of them that picked up the phone from the call center.

Table 12 Local average treatment effects associated to picking up the phone from the firm.

\begin{tabular}{|c|c|c|c|c|}
\hline & \multicolumn{4}{|c|}{ Dependent variable: } \\
\hline & \multicolumn{2}{|c|}{ RR after 120 days } & \multicolumn{2}{|c|}{ Churn by Jan 2016} \\
\hline & 2SLS & 2SLS & 2SLS & 2SLS \\
\hline & $(1)$ & $(2)$ & $(3)$ & $(4)$ \\
\hline Gets Call (b1) & $\begin{array}{c}-0.033^{* *} \\
(0.013)\end{array}$ & & $\begin{array}{c}-0.037^{* * *} \\
(0.012)\end{array}$ & \\
\hline Fraction of Alters Get Call (b2) & & $\begin{array}{c}-0.026^{* *} \\
(0.012)\end{array}$ & & $\begin{array}{c}-0.024^{* *} \\
(0.011)\end{array}$ \\
\hline Number of Alters & & $\begin{array}{l}-0.001 \\
(0.002)\end{array}$ & & $\begin{array}{c}-0.007^{* * *} \\
(0.002)\end{array}$ \\
\hline Churn Score & $\begin{array}{c}0.162^{* * *} \\
(0.040)\end{array}$ & $\begin{array}{c}0.141^{* * *} \\
(0.039)\end{array}$ & $\begin{array}{c}0.044 \\
(0.036)\end{array}$ & $\begin{array}{c}0.038 \\
(0.036)\end{array}$ \\
\hline Constant & $\begin{array}{c}0.091^{* * *} \\
(0.026) \\
\end{array}$ & $\begin{array}{c}0.085^{* * *} \\
(0.025) \\
\end{array}$ & $\begin{array}{c}0.179^{* * *} \\
(0.024) \\
\end{array}$ & $\begin{array}{c}0.179^{* * *} \\
(0.023) \\
\end{array}$ \\
\hline Period Dummies & Yes & Yes & Yes & Yes \\
\hline Stock Yogo - b1 (inst $=$ To Call Ego) & $4415.422^{* * *}$ & & $4415.422^{* * *}$ & \\
\hline Stock Yogo - b2 (inst $=$ To Call Ego and Alter $)$ & & $58085.116^{* * *}$ & & $58085.116^{* * *}$ \\
\hline Wu-Hausman & 0.031 & $5.062 * *$ & 1.693 & $3.616^{*}$ \\
\hline Wald & $7.365^{* * *}$ & $6.242^{* * *}$ & $11.732^{* * *}$ & $11.776^{* * *}$ \\
\hline Observations & 37,308 & 37,132 & 37,308 & 37,132 \\
\hline$\underline{\text { Residual Std. Error }}$ & 0.391 & 0.389 & 0.354 & 0.352 \\
\hline Note: & $\begin{array}{r}\text { Cluster Rob } \\
\text { Ob } \\
\text { (1) anc } \\
\text { (2) and (4) h }\end{array}$ & $\begin{array}{l}\text { st and Heterosk } \\
\text { ervations cluste } \\
\text { (3) have Ego h } \\
\text { ve Ego househo }\end{array}$ & $\begin{array}{l}{ }^{*} \mathrm{p}<0 \\
\text { dastic Consist } \\
\text { d by social } \mathrm{n} \\
\text { aseholds in C } \\
\text { ls in Call Ego }\end{array}$ & $\begin{array}{l}\mathrm{p}<0.05 ;{ }^{* * *} \mathrm{p}< \\
\text { andar Errors } \\
\text { treatment cly } \\
\text { One and Call } \\
\text { Call Ego and }\end{array}$ \\
\hline
\end{tabular}




\section{Appendix G: Moderating Effect of Tie Strength}

We analyzed whether tie strength and degree centrality played a moderating role in our results. Table 13 shows these results. We proxy the tie strength between an ego and her alters using two covariates number of calls and airtime - which we interact with our treatment conditions. None of these interactions are statistically significant in our setting and, therefore, close ties or number of friends do not moderate the effects observed.

Table 13 Treatment effects moderated by tie strength.

\begin{tabular}{|c|c|c|c|c|c|c|}
\hline & \multicolumn{6}{|c|}{ Dependent variable: } \\
\hline & $\begin{array}{c}\mathrm{RR} \\
120 \text { days } \\
(1)\end{array}$ & $\begin{array}{c}\text { Churn } \\
\text { Jan } 2016 \\
(2) \\
\end{array}$ & $\begin{array}{c}\mathrm{RR} \\
120 \text { days } \\
(3)\end{array}$ & $\begin{array}{c}\text { Churn } \\
\text { Jan } 2016 \\
(4) \\
\end{array}$ & $\begin{array}{c}\mathrm{RR} \\
120 \text { days } \\
(5)\end{array}$ & $\begin{array}{c}\text { Churn } \\
\text { Jan } 2016 \\
(6)\end{array}$ \\
\hline Call Alter & $\begin{array}{c}0.006 \\
(0.011)\end{array}$ & $\begin{array}{l}-0.008 \\
(0.010)\end{array}$ & $\begin{array}{c}0.006 \\
(0.010)\end{array}$ & $\begin{array}{l}-0.011 \\
(0.009)\end{array}$ & $\begin{array}{l}-0.019 \\
(0.019)\end{array}$ & $\begin{array}{l}-0.025 \\
(0.017)\end{array}$ \\
\hline Call Ego & $\begin{array}{c}-0.017^{* *} \\
(0.008)\end{array}$ & $\begin{array}{c}-0.016^{* *} \\
(0.007)\end{array}$ & $\begin{array}{c}-0.015^{* *} \\
(0.008)\end{array}$ & $\begin{aligned}-0.018^{* * *} \\
(0.007)\end{aligned}$ & $\begin{array}{l}-0.017 \\
(0.014)\end{array}$ & $\begin{array}{l}-0.012 \\
(0.013)\end{array}$ \\
\hline Call Ego and Alter & $\begin{array}{c}-0.023^{* *} \\
(0.011)\end{array}$ & $\begin{aligned}-0.027^{* * *} & (0.010)\end{aligned}$ & $\begin{aligned}-0.027^{* * *} & (0.010)\end{aligned}$ & $\begin{array}{l}-0.032^{* * *} \\
(0.009)\end{array}$ & $\begin{array}{c}-0.030^{*} \\
(0.018)\end{array}$ & $\begin{array}{l}-0.024 \\
(0.017)\end{array}$ \\
\hline Call Alter * Avg. Calls to Alters & $\begin{array}{c}-0.00004 \\
(0.0001)\end{array}$ & $\begin{array}{c}-0.00000 \\
(0.0001)\end{array}$ & & & & \\
\hline Call Ego * Avg. Calls to Alters & $\begin{array}{l}-0.00003 \\
(0.0001)\end{array}$ & $\begin{array}{l}-0.0001 \\
(0.0001)\end{array}$ & & & & \\
\hline Call Ego and Alter * Avg. Calls to Alters & $\begin{array}{l}-0.0001 \\
(0.0001)\end{array}$ & $\begin{array}{l}-0.0001 \\
(0.0001)\end{array}$ & & & & \\
\hline Call Alter $*$ Avg. Airtime to Alters & & & $\begin{array}{l}-0.0003 \\
(0.0003)\end{array}$ & $\begin{array}{c}0.0003 \\
(0.0004)\end{array}$ & & \\
\hline Call Ego * Avg. Airtime to Alters & & & $\begin{array}{l}-0.0003 \\
(0.0003)\end{array}$ & $\begin{array}{l}-0.0002 \\
(0.0003)\end{array}$ & & \\
\hline Call Ego and Alter * Avg. Airtime to Alters & & & $\begin{array}{l}-0.0003 \\
(0.0004)\end{array}$ & $\begin{array}{l}0.0001 \\
(0.0004)\end{array}$ & & \\
\hline Call Alter * Number of Alters & & & & & $\begin{array}{c}0.011 \\
(0.009)\end{array}$ & $\begin{array}{c}0.010 \\
(0.008)\end{array}$ \\
\hline Call Ego * Number of Alters & & & & & $\begin{array}{c}-0.0001 \\
(0.006)\end{array}$ & $\begin{array}{l}-0.004 \\
(0.006)\end{array}$ \\
\hline Call Ego and Alter * Number of Alters & & & & & $\begin{array}{l}-0.001 \\
(0.009)\end{array}$ & $\begin{array}{l}-0.005 \\
(0.008)\end{array}$ \\
\hline Avg. Calls to Alters & $\begin{array}{l}0.00002 \\
(0.0001)\end{array}$ & $\begin{array}{c}-0.00001 \\
(0.0001)\end{array}$ & & & & \\
\hline Avg. Airtime to Alters & & & $\begin{array}{c}0.0002 \\
(0.0003)\end{array}$ & $\begin{array}{l}-0.0001 \\
(0.0003)\end{array}$ & & \\
\hline Churn Score & $\begin{array}{c}0.120^{* * *} \\
(0.038)\end{array}$ & $\begin{array}{c}0.049 \\
(0.034)\end{array}$ & $\begin{array}{c}0.120^{* * *} \\
(0.038)\end{array}$ & $\begin{array}{c}0.050 \\
(0.034)\end{array}$ & $\begin{array}{c}0.127^{* * *} \\
(0.035)\end{array}$ & $\begin{array}{c}0.049 \\
(0.032)\end{array}$ \\
\hline Number of Alters & $\begin{array}{c}0.001 \\
(0.002)\end{array}$ & $\begin{array}{c}-0.003^{* *} \\
(0.002)\end{array}$ & $\begin{array}{c}0.001 \\
(0.002)\end{array}$ & $\begin{array}{c}-0.003^{* *} \\
(0.002)\end{array}$ & $\begin{array}{l}-0.001 \\
(0.006)\end{array}$ & $\begin{array}{l}-0.003 \\
(0.006)\end{array}$ \\
\hline Constant & $\begin{array}{c}0.101^{* * *} \\
(0.025) \\
\end{array}$ & $\begin{array}{c}0.178^{* * *} \\
(0.023)\end{array}$ & $\begin{array}{c}0.099^{* * *} \\
(0.024)\end{array}$ & $\begin{array}{c}0.178^{* * *} \\
(0.022)\end{array}$ & $\begin{array}{c}0.104^{* * * *} \\
(0.025) \\
\end{array}$ & $\begin{array}{c}0.179^{* * *} \\
(0.023)\end{array}$ \\
\hline Period Dummies & Yes & Yes & Yes & Yes & Yes & Yes \\
\hline Observations & 42,591 & 42,591 & 42,591 & 42,591 & 45,023 & 45,023 \\
\hline $\mathrm{R}^{2}$ & 0.002 & 0.003 & 0.002 & 0.003 & 0.002 & 0.003 \\
\hline Adjusted $\mathrm{R}^{2}$ & 0.002 & 0.003 & 0.002 & 0.002 & 0.002 & 0.003 \\
\hline Residual Std. Error & 0.391 & 0.352 & 0.391 & 0.352 & 0.391 & 0.355 \\
\hline F Statistic & $5.445^{* * *}$ & $8.233^{* * *}$ & $5.371^{* * *}$ & $7.512^{* * *}$ & $6.194^{* * *}$ & $10.453^{* * *}$ \\
\hline Note: & $\begin{array}{l}\text { Cluster Ro } \\
\text { vations clu } \\
\text { retention; } \\
\text { did not ha }\end{array}$ & $\begin{array}{l}\text { bust and } \mathrm{H} \\
\text { istered by so } \\
\text { Excludes } 1,0 \\
\text { ve Alters cor }\end{array}$ & $\begin{array}{l}\text { eteroskedast } \\
\text { cial network } \\
34 \text { Egos wit } \\
\text { mplete call in }\end{array}$ & $\begin{array}{l}\text { ic Consisten } \\
\text { treatment } \\
\text { h zero Alter } \\
\text { nformation }\end{array}$ & $\begin{array}{l}{ }^{*} \mathrm{p}<0.1 ; \\
\text { t } \text { Standar } \\
\text { cluster; } \mathrm{RP} \\
\text { s and } 1,398\end{array}$ & $\begin{array}{l}0.05 ;{ }^{* * *} \mathrm{p}<0.01 \\
\text { rs in (); Obser- } \\
\text { nds for reactive } \\
\text { os for whom we }\end{array}$ \\
\hline
\end{tabular}




\section{Appendix H: The Effect of Satisfaction Surveys on Consumer Retention}

This appendix shows that satisfaction surveys do not drive the results that we observe in our setting. We start with the case of egos. Consider the directed acyclic graph in Panel A of Figure 14, which includes all paths that can affect the retention of egos in our scenario. Namely, entering reactive retention may be directly caused by getting a call - which always includes a satisfaction survey - (L1), or indirectly, through the effect of getting an offer (L2). In addition, entering reactive retention may also be caused by an unobserved affecting the likelihood of getting a call, getting an offer or entering retention. We present a general case here where we assume that L1, L2 and L3 are all potentially present.

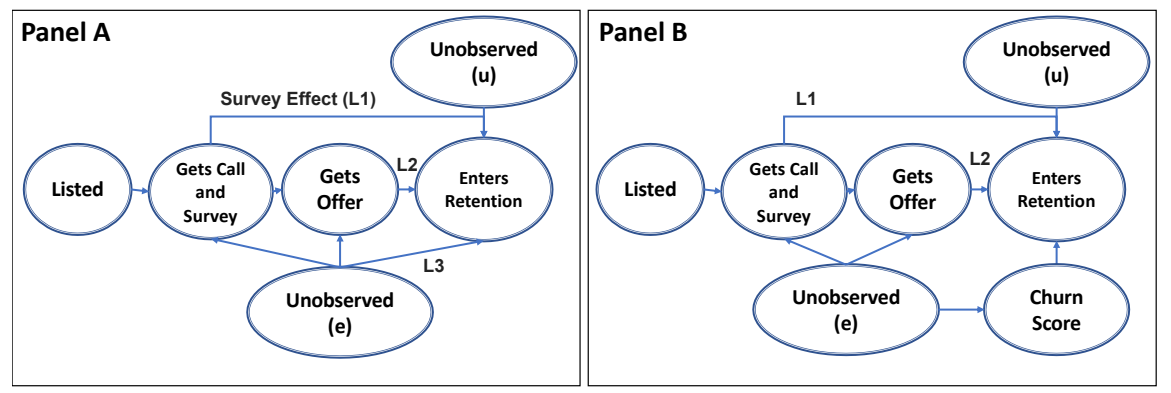

Figure 14 Directed acyclic graph and possible causal paths between getting a call, getting an offer and entering into retention.

We now focus on egos across the Call No One condition and the Call Ego condition. In the main body of the paper, we show that the latter enter reactive retention less often. In other words, we show that there is a causal effect from listing the ego to call (Listed in the graph) on entering retention through L1, L2, or both. If L3 is not present, i.e., if there is no endogeneity, one can identify L1 by regressing Enters Retention on Gets Call and Survey controlling for Gets Offer. In this case, Gets Call and Survey captures the effect through L1 while Gets Offer captures the effect through L2. However, if L3 is present, one needs to instrument Get Calls and Survey with ego Listed to call in order to block the path between the unobserved (e) and Gets Call and Survey. However, with only one instrument, one cannot empirically separate the causal effect through L1 from that through L2. To identify these effects separately one would need another instrument for Gets Offer. Alternatively, one can try to block the path between the unobservable (e) and the outcome (Enters 
Retention) using a control variable that stands between them. Given that we do not have an additional instrument in our setting for Gets Offer, we followed the second approach referred above and we tested whether controlling for Churn Score (the churn score provided by our churn prediction algorithm) is enough to block path L3, which would thus allow us to separate the effects of Gets Call and Survey through L1 and L2.

Churn Score may be a good covariate for this purpose because it was generated before the experiment started and was used to identify households likely to churn in our setting. Therefore, Churn Score is the most natural candidate covariate to consider in our setting to block the confounding effect that L3 may introduce. To test if indeed Churn Score helps identify the direct effect of Gets Call and Survey on Enters Retention, we use whether the ego was listed to be called by the firm (Listed in this figure) as an instrument for Gets Call and Survey. Recall that whether egos were listed to be called by the firm was randomized in our setting. Furthermore, we use this (randomized) instrument to test for the endogeneity of Gets Call and Survey after controlling for Churn Score.

Table 14 presents our results using egos in the Call No One condition and in the Call Ego condition, which corroborate our hypothesis that Churn Score is a good covariate in our setting to block L3. Column (1) shows the first stage results. We can see here that whether the ego was listed to be called by the firm is strongly correlated with the probability of getting the call and the satisfaction survey. Column (2) shows the second stage results, which show that egos who actually got the call from the firm churn less than egos that did not but that would have been contacted by the firm had they been listed for proactive churn management. The parameter for Gets Call in column (2) of this table captures the effect of all paths from Gets Call and Survey to Enters Retention and, as expected, is larger in magnitude than the Intent-To-Treat (ITT) estimate that we reported in the main body of the paper because it measures the effect across the sub-population of compliers (those that pick up the call from the firm). Column (3) is our test of endogeneity. We follow (Wooldridge 2010, Chapter 6, page 126) and use the residuals of the first stage regression as an additional covariate in the linear regression of Enters Retention on Gets Call and Survey. If the residuals of the first stage are statistically significant in the second stage then we have evidence of endogeneity. If they are not, we cannot reject the null hypothesis that Gets Call and Survey is exogenous in our setting after controlling for Churn Score. The latter is precisely what we find, thus increasing our confidence that in our setting Gets Call and Survey is exogenous after controlling for Churn Score. The standard errors in column (3) were generated using 500 block bootstrap replications with treatment clusters used as sampling units. Finally, column (4) is a robustness check that shows that a simple OLS of Enters Retention on Gets Call and Survey after controlling for Churn Score generates the same estimate as that obtained using two stage least squares, as we would now expect given that L3 is appropriately blocked by Churn Score. Taken together, these results provide strong evidence that Churn Score is a good covariate to block the effect through L3 in Panel A of figure 14 .

After having shown that conditioning for Churn Score is a good strategy to separate the direct effect of Gets Call and Survey from the effect of Gets Offer on Enters Retention, the next step in our analysis is to run an OLS of Enters Retention on Gets Call and Survey and on Gets Offer together controlling for Churn 
Table 14 Regressions to test the endogeneity of Gets Call and Survey using Churn Score to block L3.

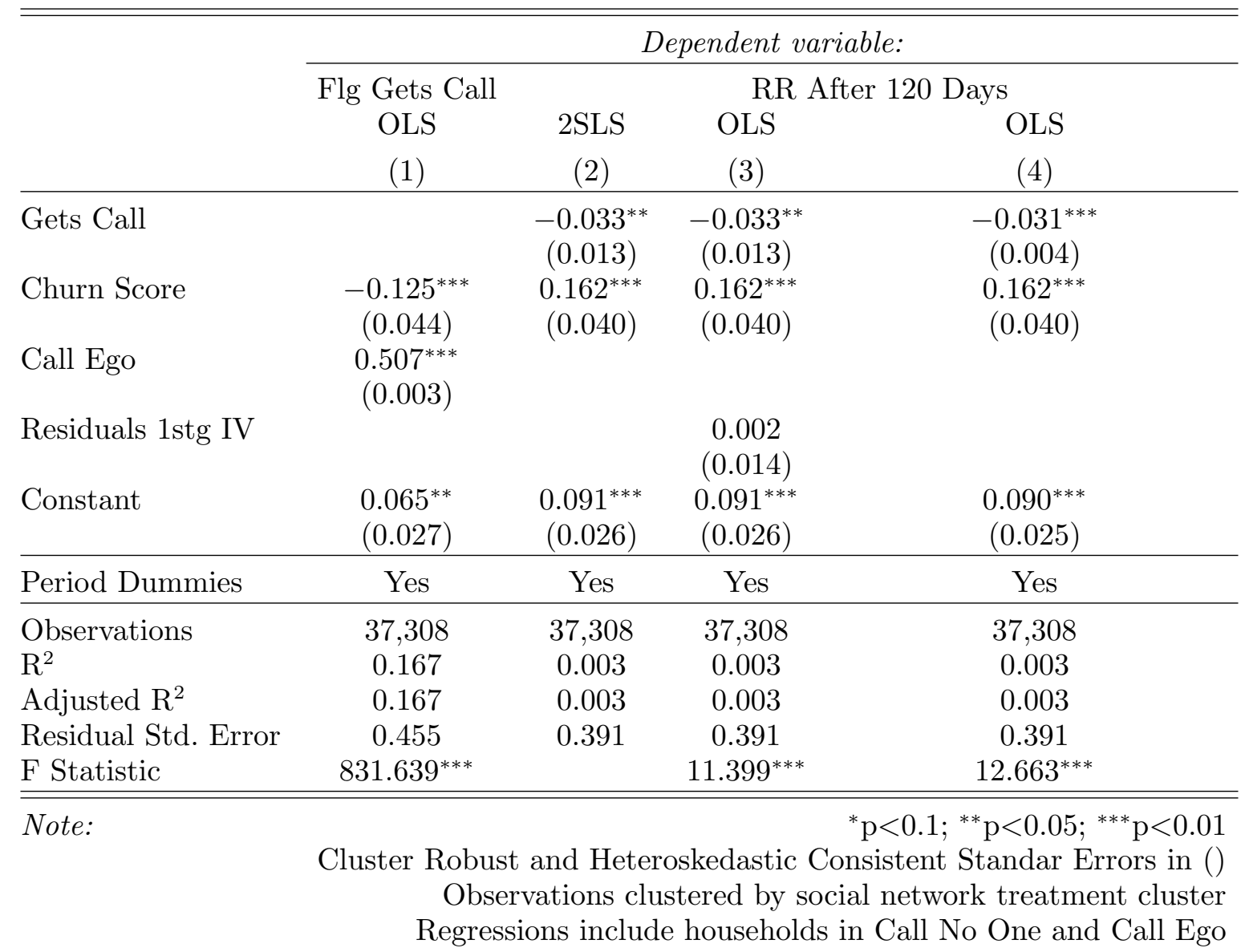

Score. Doing so allows us to separate the direct effect of the satisfaction survey (L1) from the effect of getting an offer (L2). Column (1) of Table 15 shows the results obtained. After controlling for the effect of getting an offer, the satisfaction survey has no effect on entry into retention. Column (2) in this table provides an additional robustness check. If the world works as we describe in Panel B of Figure 14, then after controlling for Gets Call and Survey and Churn Score, the effect of Gets Offer on Enters Retention is exogenous. This means that if we only analyze egos that pickup the phone (and thus go through the satisfaction survey) we must find the same effect of Gets Offer on Enters Retention as we would with the full regression that controls for both Churn score and Gets Call and Survey. This is exactly what we obtain in column (2) of this table. Taken as a whole, these results provide significant robust evidence that the effect of traditional proactive churn management in our setting works through the offers extended to egos and not through the satisfaction survey alone.

Finally, given that egos and alters are different consumers in our setting (likely and non-likely churners), one may argue that the results reported above for egos may not extend to alters. To address this concern, we repeated the analysis above now comparing alters in the Call Ego condition to alters in the Call Ego and Alter condition. In this case, we use whether the alter was listed to be contacted by the firm as an instrumental variable and we test whether controlling for the alter's churn score helps us block the path 
Table 15 Effect of Gets Offer and Gets Call and Survey on the ego's entry into retention.

\begin{tabular}{|c|c|c|}
\hline & \multicolumn{2}{|c|}{ Dependent variable: } \\
\hline & \multicolumn{2}{|c|}{ RR After 120 Days } \\
\hline & $\begin{array}{l}\text { All Households } \\
\text { (1) }\end{array}$ & $\begin{array}{l}\text { OLS } \\
\text { Households with Gets Call }=1 \\
\qquad(2)\end{array}$ \\
\hline Gets call (b1) & $\begin{array}{l}-0.001 \\
(0.006)\end{array}$ & \\
\hline Gets Offer (b2) & $\begin{array}{c}-0.045^{* * *} \\
(0.007)\end{array}$ & $\begin{array}{c}-0.046^{* * *} \\
(0.007)\end{array}$ \\
\hline Churn Score & $\begin{array}{c}0.160^{* * *} \\
(0.040)\end{array}$ & $\begin{array}{c}0.186^{* * *} \\
(0.062)\end{array}$ \\
\hline Constant & $\begin{array}{c}0.091^{* * *} \\
(0.025)\end{array}$ & $\begin{array}{l}0.078^{* *} \\
(0.038) \\
\end{array}$ \\
\hline Period Dummies & Yes & Yes \\
\hline Observations & 37,308 & 17,003 \\
\hline $\mathrm{R}^{2}$ & 0.004 & 0.008 \\
\hline Adjusted $\mathrm{R}^{2}$ & 0.004 & 0.007 \\
\hline Residual Std. Error & 0.391 & 0.379 \\
\hline F Statistic & $16.146^{* * *}$ & $14.445^{* * *}$ \\
\hline Note: & $\begin{array}{r}\text { Cluster Robust } \\
\text { Obser } \\
\text { Regressi }\end{array}$ & $\begin{array}{l}\qquad{ }^{*} \mathrm{p}<0.1 ;{ }^{* *} \mathrm{p}<0.05 ; \\
\text { skedastic Consistent Standar Er } \\
\text { tered by social network treatme } \\
\text { households in Call No One and }\end{array}$ \\
\hline
\end{tabular}

between the unobserved and the alter's entry into retention. Tables 16 and 17 show the results obtained, which are qualitatively similar to the case of egos. In addition, this table also corroborates that in our setting the rate at which alters enter retention was not affected by proactive churn management, neither through the satisfaction survey nor through the offers that they received. 
Table 16 Regressions to test the endogeneity of the Gets Call and Survey conditional on Churn Score for the case of alters.

\begin{tabular}{|c|c|c|c|c|}
\hline & \multicolumn{4}{|c|}{ Dependent variable: } \\
\hline & \multirow{2}{*}{$\begin{array}{c}\text { Flg Gets Call } \\
\text { OLS }\end{array}$} & \multicolumn{3}{|c|}{ RR After 120 Days } \\
\hline & & 2SLS & OLS & OLS \\
\hline & $(1)$ & $(2)$ & $(3)$ & $(4)$ \\
\hline \multirow[t]{2}{*}{ Gets Call } & & -0.010 & -0.010 & -0.007 \\
\hline & & $(0.007)$ & $(0.008)$ & $(0.005)$ \\
\hline \multirow{2}{*}{ Churn Score } & $-0.008^{* * *}$ & $0.118^{* * *}$ & $0.118^{* * *}$ & $0.118^{* * *}$ \\
\hline & $(0.003)$ & $(0.006)$ & $(0.006)$ & $(0.006)$ \\
\hline \multirow[t]{2}{*}{ Flg No Churn Score } & $-0.015^{* * *}$ & $0.040^{* * *}$ & $0.040^{* * *}$ & $0.040^{* * *}$ \\
\hline & $(0.002)$ & $(0.004)$ & $(0.004)$ & $(0.004)$ \\
\hline \multirow{2}{*}{ Call Alter } & $0.581^{* * *}$ & & & \\
\hline & $(0.007)$ & & & \\
\hline \multirow[t]{2}{*}{ Residuals 1stg IV } & & & 0.007 & \\
\hline & & & $(0.011)$ & \\
\hline \multirow{2}{*}{ Constant } & 0.001 & $0.104^{* * *}$ & $0.104^{* * *}$ & $0.103^{* * *}$ \\
\hline & $(0.003)$ & $(0.005)$ & $(0.005)$ & $(0.005)$ \\
\hline Period Dummies & Yes & Yes & Yes & Yes \\
\hline Observations & 61,968 & 61,968 & 61,968 & 61,968 \\
\hline $\mathrm{R}^{2}$ & 0.560 & 0.008 & 0.008 & 0.008 \\
\hline Adjusted $\mathrm{R}^{2}$ & 0.560 & 0.008 & 0.008 & 0.008 \\
\hline Residual Std. Error & 0.153 & 0.315 & 0.315 & 0.315 \\
\hline F Statistic & $7,877.330^{* * *}$ & & $43.639^{* * *}$ & $47.962^{* * *}$ \\
\hline
\end{tabular}

Note: $\quad{ }^{*} \mathrm{p}<0.1 ;{ }^{* *} \mathrm{p}<0.05 ;{ }^{* * *} \mathrm{p}<0.01$

Cluster Robust and Heteroskedastic Consistent Standar Errors in ()

Observations clustered by social network treatment cluster Regressions include Alter households in Call Ego and Call Ego and Alter 
Table 17 Effect of Gets Offer and Gets Call and Survey on the rate at which alters enter into retention.

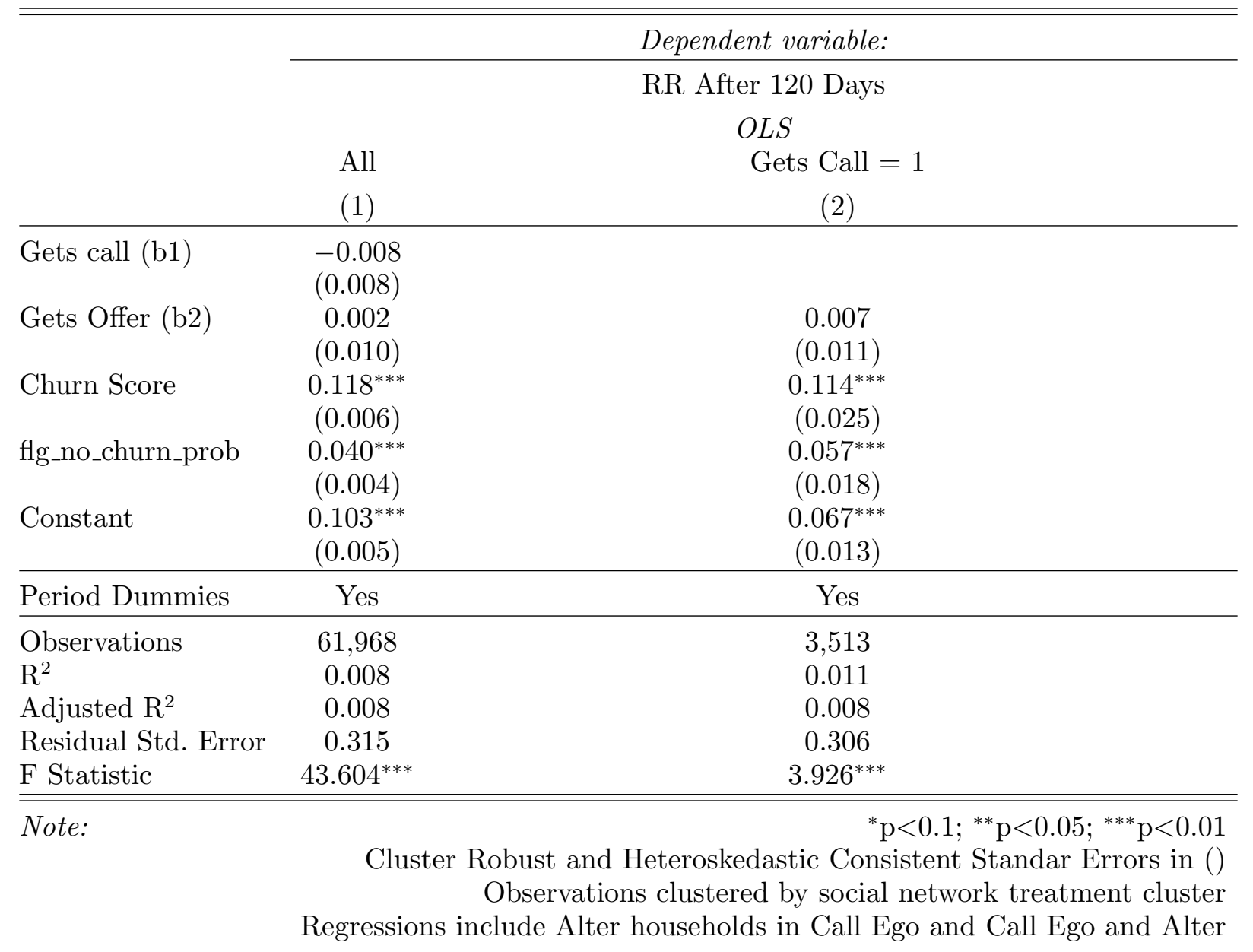




\section{Appendix I: Inability to Separate Effects of Alters on Egos Entry into Retention}

Consider egos and alters in the Call Ego and the Call Ego and Alters conditions. Panel A in Figure 15

illustrates the causal paths that may link to the decision of egos to enter into reactive retention in our context.
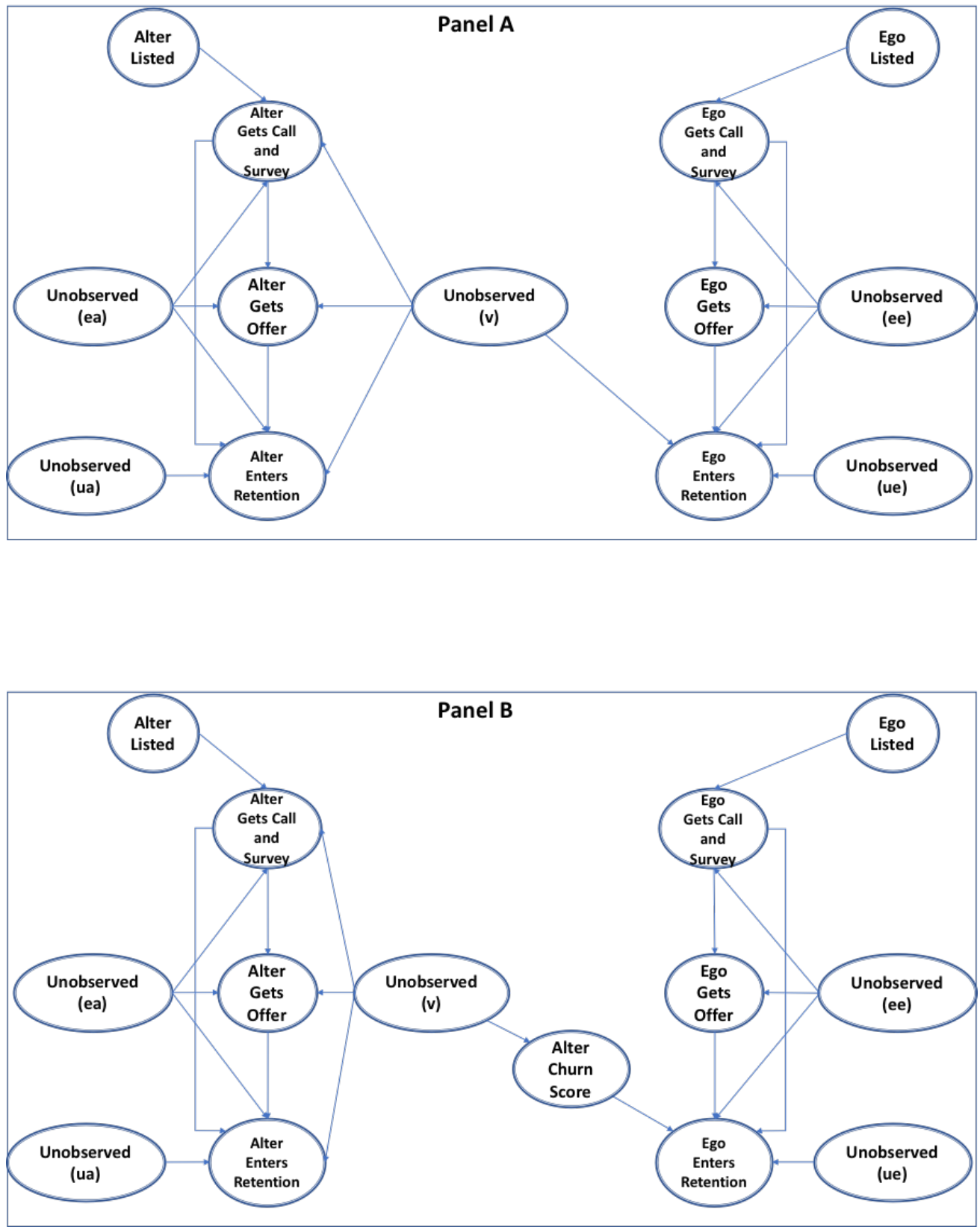

Figure 15 Causal paths for socially based proactive churn management 
We do not include direct links from Alter Gets Call and Survey, Alter Gets Offer and Alter Enters Retention towards Ego Gets Call and Survey or Ego Gets Offer in this graph because we have extensive empirical evidence (all reported in the main body of the paper) that egos in the Call Ego and in the Call Ego and Alter conditions picked up the phone at the same rate, received offers at the same rate and, in fact, received the same offers at the same rates (but enter reactive retention at different rates). Table 8 in the paper shows these results. We also omit links from the ego's covariates to the alters' covariates because we have no empirical evidence that egos influence alters in our setting. First, we have evidence that, on average, alters are contacted 4.9 days earlier than egos (the $95 \%$ confidence interval for this statistic is [ $5.698 ;-4.228]$ days). Second, when we compare alters in the Call Alter condition to alters in the Call Ego and Alter condition we find that they picked up the phone from the firm at the same rate, received offers with the same probability, accepted offers at the same rate and entered reactive retention at the same rate (see table 18). Therefore, we have no empirical evidence that egos influence the decisions of alters at all in our setting.

Table 18 Effect of assignement on Alter outcomes

\begin{tabular}{|c|c|c|c|}
\hline & \multicolumn{3}{|c|}{ Dependent variable: } \\
\hline & $\begin{array}{c}\text { Get Call } \\
(1)\end{array}$ & $\begin{array}{c}\text { Get Offer } \\
(2)\end{array}$ & $\begin{array}{c}\text { RR } 120 \text { days } \\
(3)\end{array}$ \\
\hline Call Ego and Alter & $\begin{array}{c}0.013 \\
(0.009)\end{array}$ & $\begin{array}{c}0.002 \\
(0.008)\end{array}$ & $\begin{array}{c}0.005 \\
(0.006)\end{array}$ \\
\hline Churn Score & $\begin{array}{c}0.031^{*} \\
(0.019)\end{array}$ & $\begin{array}{c}0.181^{* * *} \\
(0.018)\end{array}$ & $\begin{array}{c}0.062^{* * *} \\
(0.012)\end{array}$ \\
\hline Constant & $\begin{array}{c}0.566^{* * *} \\
(0.014)\end{array}$ & $\begin{array}{c}0.298^{* * *} \\
(0.012)\end{array}$ & $\begin{array}{c}0.098^{* * *} \\
(0.008)\end{array}$ \\
\hline Period Dummies & Yes & Yes & Yes \\
\hline $\begin{array}{l}\text { Observations } \\
\mathrm{R}^{2} \\
\text { Adjusted } \mathrm{R}^{2} \\
\text { Residual Std. Error } \\
\text { F Statistic }\end{array}$ & $\begin{array}{c}12,278 \\
0.069 \\
0.068 \\
0.477 \\
100.786^{* * *} \\
\end{array}$ & $\begin{array}{c}12,278 \\
0.043 \\
0.042 \\
0.450 \\
61.083^{* * *} \\
\end{array}$ & $\begin{array}{c}12,278 \\
0.003 \\
0.002 \\
0.306 \\
4.149^{* * *}\end{array}$ \\
\hline Note: & $\begin{array}{l}\text { Cluster R } \\
\text { Standard I } \\
\text { social net } \\
\text { include Alt }\end{array}$ & $\begin{array}{l}{ }^{*} \mathrm{p}<0.1 \\
\text { ad Heteros } \\
() ; \text { Observ } \\
\text { eatment } \\
\text { ll Alter an }\end{array}$ & $\begin{array}{l}05 ;{ }^{* *} \mathrm{p}<0.01 \\
\text { Consistent } \\
\text { clustered by } \\
\text { Regressions } \\
\text { go and Alter }\end{array}$ \\
\hline
\end{tabular}

We start with the regression below to try to measure the effects of Alter Gets Call and Survey, Alter Gets Offer and Alter Enters Retention on Ego Enters Retention, where Fraction of Alters Get Call and Survey indicates, for each ego, the percentage of her alters that pick up the phone from the firm and thus receive the satisfaction survey. Similar definitions apply for the Fraction of Alter Get Offers and for the Fraction of Alters Enter Reactive Retention: 


\section{EgoEntersRetention $=\alpha_{1}+\alpha_{2}$ Fractionof AltersGetCallandSurvey + $\alpha_{3}$ Fractionof AltersGetOffers $+\alpha_{4}$ Fractionof AltersEnterReactiveRetention $+u$}

However, it might be that some or all of the right hand side variables in this regression are correlated with unobservables that also determine the ego's entry to retention (because, for example, of social network homophily). Therefore, these variables are potentially endogenous. One way to address this concern is to obtain three instrumental variables, one for each of these potentially endogenous covariates, that we could use simultaneously. Another way to address this concern is to follow the same approach that we employed in appendix $\mathrm{H}$ for egos and alters separately and find a good proxy that may block the paths between these covariates and ego's entry into retention. Unfortunately, in this case, we were unable to find suitable instruments in addition to the random assignment of egos and alters to treatment conditions (which does not provide us with enough instruments in our casse). Therefore, we tried to use the average churn score of alters, determined by our machine learning algorithm based on data prior to the experiment, to block the paths between the right hand side variables in this regression and the ego's entry into retention.

We use whether the alters were listed to be called by the firm as an instrument for Fraction of Alters Get Call and Survey to test whether the average churn score of alters helps us identify the direct effect of the Fraction of Alters Get Call and Survey on Ego Enters Retention. Recall that whether alters were listed to be called by the firm was randomized in our setting. Furthermore, we also use this instrument to test for the endogeneity of the Fraction of Alters Get Call and Survey after controlling for the average churn score of alters. Table I presents the results obtained using egos in the Call Ego and in the Call Ego and Alter conditions. These results fail to corroborate our hypothesis that the average churn score of alters is a good variable in our setting to help us separate the effect of the Fraction of Alters Get Call from the effect of the Fraction of Alters Get Offer on the ego's likelihood OF entering into retention.

Column (1) shows the first stage results. As expected, whether alters were listed to be called by the firm is strongly correlated with their probability of picking up the phone and thus from getting the satisfaction survey. Column (2) shows the second stage results. The result in this column shows that the more alters that get calls from the firm the less the ego churns. The parameter for the Fraction of Alters Get Call and Survey in this column captures the causal effect of this covariate on the ego's likelihood of entering retention through all paths connecting the former to the latter and, as expected, this effect is larger in magnitude than the intent-to-treat estimate that we report in the paper because it measures the effect across the sub-population of compliers (the egos whose alters pick up the call from the firm).

Column (3) provides the results for our test of endogeneity. We follow (Wooldridge 2010, page 129) and use the residuals of the first stage regression as an additional covariate in the linear regression of Ego Enters Retention on the Fraction of Alters Get Call and Survey. If the residuals of the first stage are statistically significant in the second stage then we have evidence of endogeneity. If they are not, we cannot reject the null hypothesis that the Fraction of Alters Get Call and Survey is exogenous after controlling for the average churn score of alters. In this case, the residuals of the first stage are statistically significant in the second 
Table 19 Regressions for testing the endogeneity of the Fraction Gets Call variable conditional on Average Alter Churn Score control variable for alters

\begin{tabular}{|c|c|c|c|c|}
\hline & \multicolumn{4}{|c|}{ Dependent variable: } \\
\hline & \multirow{3}{*}{$\begin{array}{c}\text { Flg Gets Call } \\
\text { OLS } \\
(1)\end{array}$} & \multicolumn{3}{|c|}{ RR After 120 Days } \\
\hline & & 2SLS & OLS & OLS \\
\hline & & $(2)$ & $(3)$ & $(4)$ \\
\hline Call Ego and Alter & $\begin{array}{c}0.574^{* * *} \\
(0.007)\end{array}$ & & & \\
\hline Fraction of Friends Get Offer (b2) & $\begin{array}{l}-0.001 \\
(0.001)\end{array}$ & $\begin{array}{c}-0.002 \\
(0.002)\end{array}$ & $\begin{array}{l}-0.002 \\
(0.002)\end{array}$ & $\begin{array}{l}-0.002 \\
(0.002)\end{array}$ \\
\hline N Alters & $\begin{array}{l}-0.002 \\
(0.003)\end{array}$ & $\begin{array}{l}-0.003 \\
(0.010)\end{array}$ & $\begin{array}{l}-0.003 \\
(0.010)\end{array}$ & $\begin{array}{l}-0.003 \\
(0.010)\end{array}$ \\
\hline Avg. Alter Churn Score & & $\begin{array}{c}-0.030^{* *} \\
(0.012)\end{array}$ & $\begin{array}{c}-0.030^{* * *} \\
(0.012)\end{array}$ & $\begin{array}{l}-0.012 \\
(0.010)\end{array}$ \\
\hline Residuals 1stg IV & & & $\begin{array}{c}0.049^{* *} \\
(0.020)\end{array}$ & \\
\hline Constant & $\begin{array}{c}0.004 \\
(0.004)\end{array}$ & $\begin{array}{c}0.174^{* * *} \\
(0.009)\end{array}$ & $\begin{array}{c}0.174^{* * *} \\
(0.009)\end{array}$ & $\begin{array}{c}0.172^{* * *} \\
(0.009)\end{array}$ \\
\hline Period Dummies & Yes & Yes & Yes & Yes \\
\hline Observations & 35,012 & 35,012 & 35,012 & 35,012 \\
\hline $\mathrm{R}^{2}$ & 0.636 & 0.001 & 0.001 & 0.001 \\
\hline Adjusted $\mathrm{R}^{2}$ & 0.635 & 0.001 & 0.001 & 0.001 \\
\hline Residual Std. Error & 0.130 & 0.388 & 0.388 & 0.388 \\
\hline F Statistic & $6,105.111^{* * *}$ & & $4.202^{* * *}$ & $4.021^{* * *}$ \\
\hline Note: & $\begin{array}{l}\text { Cluster Robust } \\
\text { Standar Errors } \\
\text { social network } \\
\text { includes housel } \\
\text { and Alter }\end{array}$ & $\begin{array}{l}\quad{ }^{*} \mathrm{p}<0 . \\
\text { and Hete } \\
\text { in ();Obs } \\
\text { treatmen } \\
\text { iolds in C }\end{array}$ & $\begin{array}{l}{ }^{* *} \mathrm{p}<0.05 \text {; } \\
\text { skedastic } \\
\text { vations clu } \\
\text { cluster; R } \\
\text { ll Ego and }\end{array}$ & $\begin{array}{l}{ }^{* *} \mathrm{p}<0.01 \\
\text { onsistent } \\
\text { stered by } \\
\text { gressions } \\
\text { Call Ego }\end{array}$ \\
\hline
\end{tabular}

stage regression. Therefore, in our setting, the Fraction of Alters Get Call and Survey is still endogenous after controlling for the average churn score of alters, which precludes us from including all the potential endogenous variables in our regression at the same time to separate their causal effects. The standard errors in column (3) were generated using 500 block bootstrap replications with treatment clusters used as sampling units.

Finally, column (4) is a robustness check that shows that a simple OLS of Ego Enters Retention on the Fraction of Alters Get Call and Survey after controlling for the average churn score of alters generates estimates that are different from those obtained using two stage least squares, as we would now expect given that the Fraction of Alters Get Call is still endogenous variable. Taken together, our results show that in our setting the behavior of alters affects the decisions of egos but we cannot separate this effect into one associated to the alters that get offers from that associated to the alters that only get a call and the satisfaction survey. 


\section{Appendix J: Client Lifetime Valuation}

Blattberg et al. (2008) proposes a framework to evaluate the value associated to proactive churn management. This framework shows how one should use Customer Lifetime Value (CLV) to appropriately measure the effect of churn management initiatives and provides intuition for why proactive churn management can backfire. According to the author, the value of proactive churn management is given by $N\{\beta \gamma(C L V-\delta)+$ $(1-\beta) \psi(\Delta C L V-\delta)\}-N c$, where $N$ is the number of consumers called by the firm, $\beta$ is the probability of a churner, $\gamma$ is the likelihood of rescuing a churner, $\delta$ is the incentive given to consumers, $\psi$ is the probability that a non-churner takes the incentive and $\Delta$ is the percentage change in the CLV of non-churners that take the incentive. Therefore, the revenues associated to proactive churn management include two components - one pertains to the rescue effect on churners $\beta \gamma(C L V-\delta)$, which is likely positive given that $C L V$ is typically larger than $\delta$; the other pertains to the delight effect on non-churners $(1-\beta) \psi(\Delta C L V-\delta)$, which is likely negative given that $\triangle C L V$ is typically smaller than $\delta$. Hence, the expression above highlights clearly the tradeoff at stake with proactive churn management and shows that this strategy hurts the firm when the incentives given out to delight non-churners supersede the profit obtained from rescuing churners. The firm would like to avoid delighting non-churners but lack of precision in estimating who is a likely churner may lead it to delight too many consumers. The profit associated to proactive churn management is then obtained by subtracting the cost of the intervention from the revenues generated. The former is given by $N c$, where $c$ is the cost of calling a consumer, which is usually negligible compared to monthly bills in triple-play markets.

With individual level data from a randomized control trial such as in our case, measuring the effect of churn management on firm profit is best (e.g. more accurately) accomplished by directly computing the effect of treatment on the monthly bill and on the expected survival rate of each and every consumer intervened and combine them using the appropriate discount rate to compute the CLV of each consumer and only then aggregate across consumers to find the total effect of proactive churn management. We do so below for the cases of both proactive churn management and socially-based proactive churn management still resorting to the concepts of rescue and delight as put forward by Blattberg et al. (2008). In fact, we extend this framework to consider the potential effects of spillovers between egos and alters.

J.0.1. The Value of Proactive Churn Management With a randomized control trial, the value of proactive churn management for the firm is measured by computing the difference in the profit of the firm between calling egos and calling no one given that the latter represents the profit of the firm under reactive churn management. The following shows how to compute this difference for cluster $C$ in the social network (below $E_{g o s_{C}}$ represents the set of egos in cluster $C$ and Alters $\left(E_{g o s}\right)$ represents the alters of egos in cluster $C$ ):

$$
\begin{aligned}
& \left.\Pi_{C} \text { (Proactive Churn Management }\right)-\Pi_{C}(\text { Reactive Churn Management }) \\
& =\sum_{g \in E g s_{C}} \sum_{t=0}^{\infty} \frac{S_{g}^{t}(\text { call ego }) M B_{g}^{t}(\text { call ego })-S_{g}^{t}(\text { call no one }) M B_{g}^{t}(\text { call no one })}{(1+r)^{t}}
\end{aligned}
$$




$$
\begin{aligned}
& +\sum_{h \in \operatorname{Alters}\left(E_{g o s_{C}}\right)} \sum_{t=0}^{\infty} \frac{\left.\left.\left.S_{h}^{t} \text { (call ego }\right) M B_{h}^{t} \text { (call ego }\right)-S_{h}^{t}(\text { call no one }) M B_{h}^{t} \text { (call no one }\right)}{(1+r)^{t}} \\
& =\sum_{g \in \text { Egos }_{C}} \sum_{t=0}^{\infty} \frac{S_{g}^{t}(\text { call no one }) M B_{g}^{t}(\text { call no one })\left[\alpha_{g}^{t} \beta_{g}^{t}-1\right]}{(1+r)^{t}} \\
& +\sum_{h \in \text { Alters }\left(E_{g o s_{C}}\right)} \sum_{t=0}^{\infty} \frac{\left.S_{h}^{t} \text { (call no one }\right) M B_{h}^{t}(\text { call no one })\left[\alpha_{h}^{t} \beta_{h}^{t}-1\right]}{(1+r)^{t}}
\end{aligned}
$$

where $S_{j}^{t}(T)$ and $M B_{j}^{t}(T)$ represent the probability of survival and the monthly bill of consumer $j$ at time $t$ given treatment $T$, respectively. We also have $\alpha_{j}^{t}=S_{j}^{t}($ call ego $) / S_{j}^{t}\left(\right.$ call no one) and $\beta_{j}^{t}=$ $M B_{j}^{t}$ (call ego) $/ M B_{j}^{t}$ (call no one) as the percentage change in the probability of survival and in the monthly bill of consumer $j$ at time $t$, respectively, from reactive churn management to proactive churn management. Expression 2 measures the direct effect of proactive churn management on the egos - the effect of calling egos on themselves) - and expression 3 measures the spillover effect of proactive churn management on the alters - the effect of calling egos on alters. The former effect can be further split into two interesting terms:

$$
\begin{aligned}
& \sum_{g \in \text { Egos }_{C}} \sum_{t=0}^{\infty} \frac{\left.S_{g}^{t} \text { (call no one }\right) M B_{g}^{t}(\text { call no one })\left[\alpha_{g}^{t} \beta_{g}^{t}-1\right]}{(1+r)^{t}} \\
= & \sum_{g \in \text { Egos }_{C} \mid \text { churners }} \sum_{t=0}^{\infty} \frac{S_{g}^{t}(\text { call ego }) M B_{g}^{t}(\text { call ego })\left[1-1 /\left(\alpha_{g}^{t} \beta_{g}^{t}\right)\right]}{(1+r)^{t}} \\
+ & \sum_{g \in \text { Egos }_{C} \mid \text { non-churners }} \sum_{t=0}^{\infty} \frac{S_{g}^{t}(\text { call no one }) M B_{g}^{t}(\text { call no one })\left[\alpha_{g}^{t} \beta_{g}^{t}-1\right]}{(1+r)^{t}}
\end{aligned}
$$

Expression 4 captures the rescue effect discussed in Blattberg et al. (2008). This term tends to be positive given that both $\alpha_{j}^{t}$ and $\beta_{j}^{t}$ tend to infinity for churners because in this case both $S_{j}^{t}$ (call no one) and $M B_{j}^{t}$ (call no one) tend to zero. Expression 5 captures the delight effect discussed in Blattberg et al. (2008). This term tends to be negative because $\alpha_{j}^{t}$ tends to one (by definition, non-churners have high survival rates irrespective of treatment assignment) and $\beta_{j}^{t}$ is usually less than one (the firm reduces monthly bills to delight egos). Therefore, the tradeoff between these expressions illustrates why proactive churn management can backfire. Egos in expression 4 are true positives, which can be rescued, while egos in expression 5 are false positives, which can be delighted. Too many of the latter may lead the firm to lose profit under proactive churn management. Even if the rescue effect supersedes the delight effect on egos the firm still needs to account for the spillover effect on alters (expression 3). As discussed before when we look at the mechanisms that may be at play in our setting, this term might sometimes be negative. In our setting, we find that calling egos does not change the churn rate of alters, which likely leads to little change in their CLV.

J.0.2. The Additional Value of Socially-Based Proactive Churn Management With a randomized control trial, the additional value of socially-based proactive churn management relative to proactive churn management for the firm is measured by computing the difference in the profit of the firm between calling egos and alters and calling egos. The following shows how to compute this difference for cluster $C$ in the social network network: 
$\Pi_{C}($ Socially-Based Proactive Churn Management $)-\Pi_{C}$ (Proactive Churn Management)

$$
\begin{aligned}
& =\sum_{g \in E \text { gos }_{C}} \sum_{t=0}^{\infty} \frac{\left.\left.S_{g}^{t} \text { (call ego and alters }\right) M B_{g}^{t}(\text { call ego and alters })-S_{g}^{t}(\text { call ego }) M B_{g}^{t} \text { (call ego }\right)}{(1+r)^{t}} \\
& +\sum_{h \in \text { Alters }\left(E \text { Egos }_{C}\right)} \sum_{t=0}^{\infty} \frac{\left.\left.S_{h}^{t} \text { (call ego and alters }\right) M B_{h}^{t}(\text { call ego and alters })-S_{h}^{t}(\text { call ego }) M B_{h}^{t} \text { (call ego }\right)}{(1+r)^{t}} \\
& =\sum_{g \in E_{\text {gos }} C} \sum_{t=0}^{\infty} \frac{S_{g}^{t}(\text { call ego }) M B_{g}^{t}(\text { call ego })\left[\gamma_{g}^{t} \delta_{g}^{t}-1\right]}{(1+r)^{t}} \\
& +\sum_{h \in \text { Alters }\left(E_{\text {gos }}\right)} \sum_{t=0}^{\infty} \frac{\left.S_{h}^{t} \text { (call ego }\right) M B_{h}^{t}(\text { call ego })\left[\gamma_{h}^{t} \delta_{h}^{t}-1\right]}{(1+r)^{t}}
\end{aligned}
$$

where $\gamma_{j}^{t}=S_{j}^{t}$ (call ego and alters) $/ S_{j}^{t}$ (call ego) and $\delta_{j}^{t}=M B_{j}^{t}$ (call ego and alters) $/ M B_{j}^{t}$ (call ego) represent the percentage change in the probability of survival and in the monthly bill of consumer $j$ at time $t$, respectively, from proactive churn management to socially-based proactive churn management. Expression 6 measures the spillover effect of socially-based proactive churn management on egos - the additional effect on egos from calling alters under this strategy relative to not calling them under traditional proactive churn management - and expression 7 measures the direct effect of socially-based proactive churn management on alters - the effect on alters from calling them under this strategy relative to not calling them under traditional proactive churn management. In this case, it becomes interesting to split the latter effect into two terms:

$$
\begin{aligned}
& \quad \sum_{h \in \text { Alters }\left(\text { Egos }_{C}\right)} \sum_{t=0}^{\infty} \frac{\left.S_{h}^{t} \text { (call ego }\right) M B_{h}^{t}(\text { call ego })\left[\gamma_{h}^{t} \delta_{h}^{t}-1\right]}{(1+r)^{t}} \\
& =\sum_{h \in \text { Alters }\left(\text { Egos }_{C}\right) \mid \text { churners }} \sum_{t=0}^{\infty} \frac{S_{h}^{t}(\text { call ego and alters }) M B_{h}^{t}(\text { call ego and alters })\left[1-1 /\left(\gamma_{h}^{t} \delta_{h}^{t}\right)\right]}{(1+r)^{t}} \\
& +\sum_{h \in \text { Alters }\left(\text { Egos }_{C}\right) \mid \text { non-churners }} \sum_{t=0}^{\infty} \frac{S_{h}^{t}(\text { call ego }) M B_{h}^{t}(\text { call ego })\left[\gamma_{h}^{t} \delta_{h}^{t}-1\right]}{(1+r)^{t}}
\end{aligned}
$$

Expression 8 measures the rescue effect on alters. This term tends to be positive because both $S_{h}^{t}$ and $M B_{h}^{t}$ tend to zero for churners (and thus $\gamma_{h}^{t}$ and $\delta_{h}^{t}$ tend to infinity). Expression 9 measures the delight effect on alters. This term tends to be negative because $\gamma_{h}^{t}$ tends to one (non-churners have large survivals rates independent of treatment assignment) but $\delta_{h}^{t}$ is likely less than one because the company delights alters by offering them lower monthly bills compared to their monthly bills under the Call Ego condition when alters are not called by the firm. The aggregate of these two effects is then traded-off with the spillover effect on egos (expression 6). Therefore, our expression for the additional value of socially-based proactive churn management relative to traditional proactive churn management shows that the former strategy may work well for the firm when spillover effects are sufficiently strong and in particular when they supersede the delight effect on alters. The rescue effect on alters is likely to be very small given the very low incidence 
of likely churners among the consumers predicted as non-likely churners by the machine learning algorithms typically used to identify the former. According to our discussion above on the mechanisms that are likely at play in our setting we note that the firm may actually strengthen the spillover effect from alters to egos when it avoids delighting alters because this gives egos the additional signal that they may become on-price if they accept the offers from the firm. Understanding this dynamics of customer churn that arise in socially-rich environments and the link between what is offered and not offered to the friends of likely churners and their decisions are fundamental pieces of knowledge for churn managers in the industry that our paper analyzes.

\section{J.1. Empirical Results}

In Section 5.2 we discuss how our experiment affected the monthly bill of households. However, our experiment also changed their lock-in periods. All offers made by the firm during our experiment required households to extend their lock-in period for 1 year. This, in turn, changed their survival rates. Therefore, our analysis of how our treatments affected the CLV of the households included in our experiment proceeds in three steps. First, we determine the effect of our interventions on the expected survival rate of each household. Second, we combine these estimates with the observed changes in monthly bills to compute each household's expected CLV. Finally, we regress CLV on treatment assignment to conclude how our experiment affected the firm's bottomline.

J.1.1. Effect on Survival Rates We model the expected survival with TELCO of households in the experiment using probabilistic continuous time models, which allow for churn rates to change over time (Fader and Hardie 2007, Schweidel et al. 2008). We use the number of months elapsed between treatment assignment and churn for households that are still with the firm in January 2016 as our dependent variable. Table 20 shows the results obtained. In all cases we find that the expected survival rate of egos does not change when only alters are listed to be called by the firm. The expected survival of egos increases they are listed to be called by the firm and much more so when their alters are also listed to the called by the firm, as indicated by columns (5)-(8). The latter is a direct consequence of the lower churn rate across egos under the Call Ego and Alters condition compared to the Call Ego condition, or in other words, of the increased ability of the firm to rescue egos when their alters are also listed to be called. Panels (a) and (b) in Figure 16 plot the actual and the estimated probability of survival of egos in our sample, respectively, using the average churn score across our sample (0.7) and the estimates provided by our log-logistic model, which we use in the remainder of our paper because they yield the lowest AIC and log likelihood across the ones reported in Table 20. The dashed horizontal line represents January 2016, the last month for which we have data. The actual and the projected probability of survival for egos in our sample in January 2016 are identical, which provides some evidence that our survival model fits the data well. We also observe that in our setting, the effect of our treatments is essentially to increase survival rates right after treatment assignment. A few months after treatment assignment the likelihood of survival for treated households decays just as if they had not been treated (the survival curves become parallel). As Figure 16 shows, our treatments displaced the survival curves only vertically. This means that the effect of proactive churn management on survival rates is imminent in our setting and, due to this initial displacement, the survival rates of treated consumers remain above those of non-treated consumers throughout time. Table 21 also shows that the survival rate of 
alters does not change with treatment assignment. This is a direct consequence of the fact that alters in our sample are not likely churners.

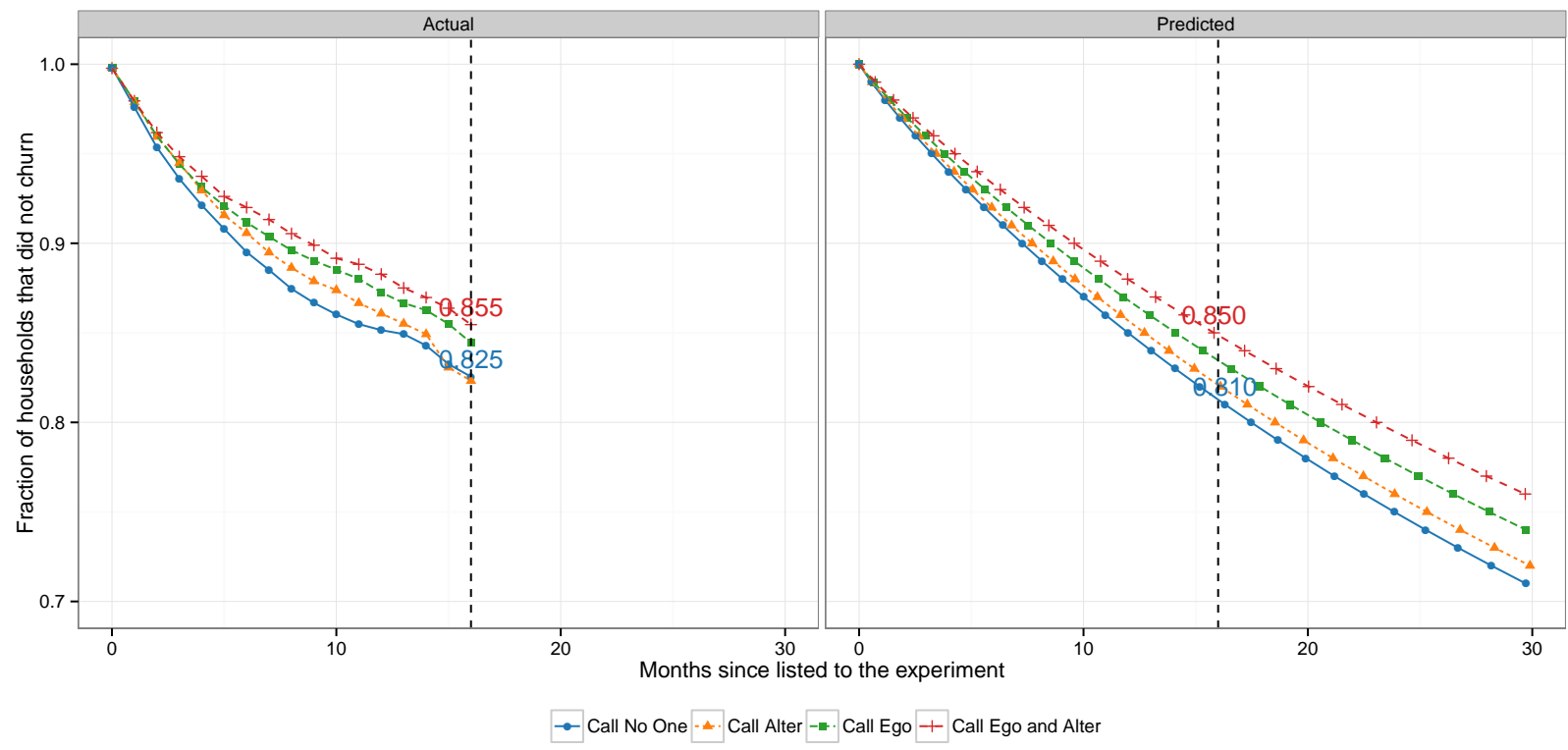

Figure 16 Actual and estimated probability of survival for egos in our experiment. 


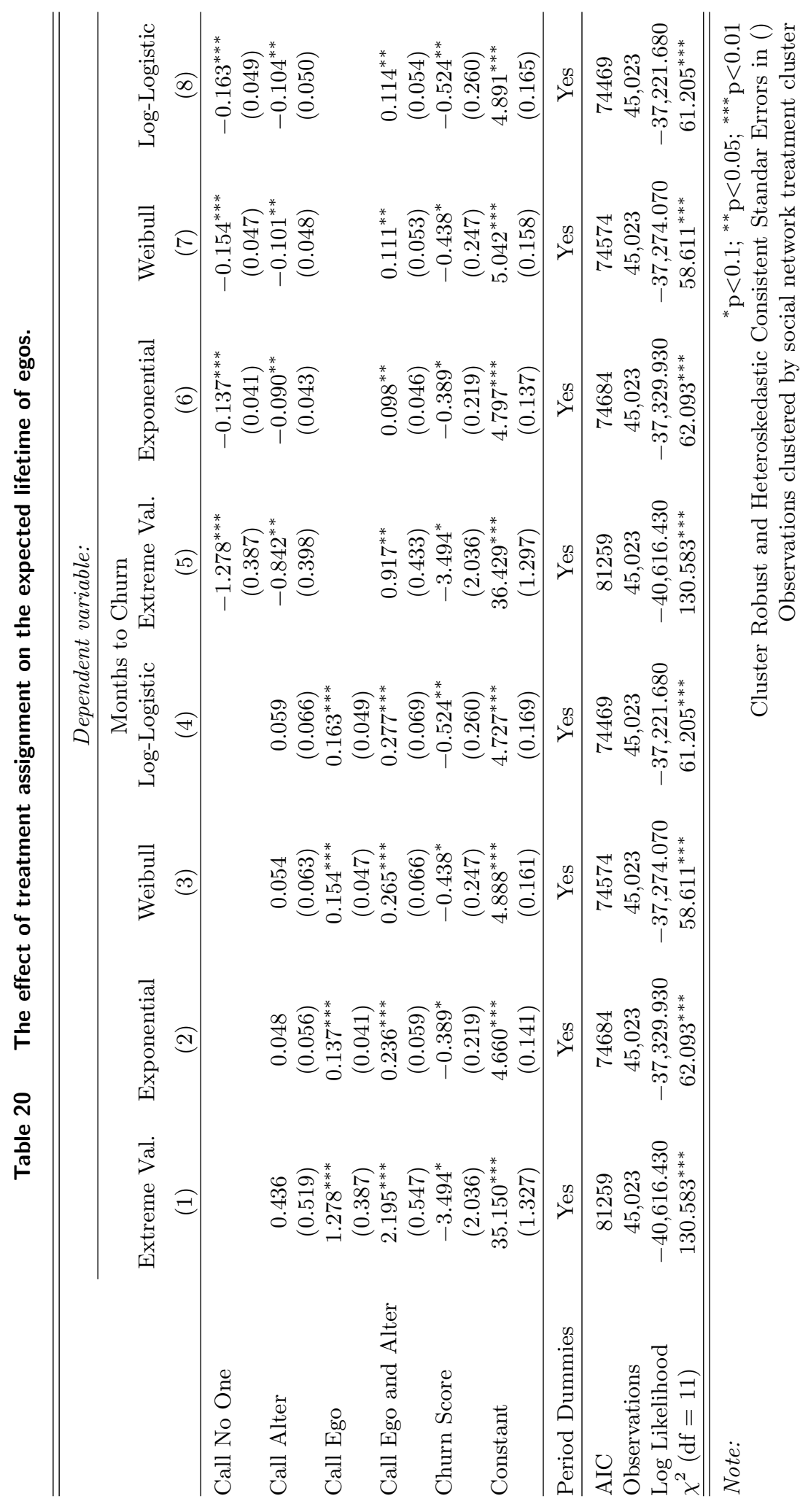




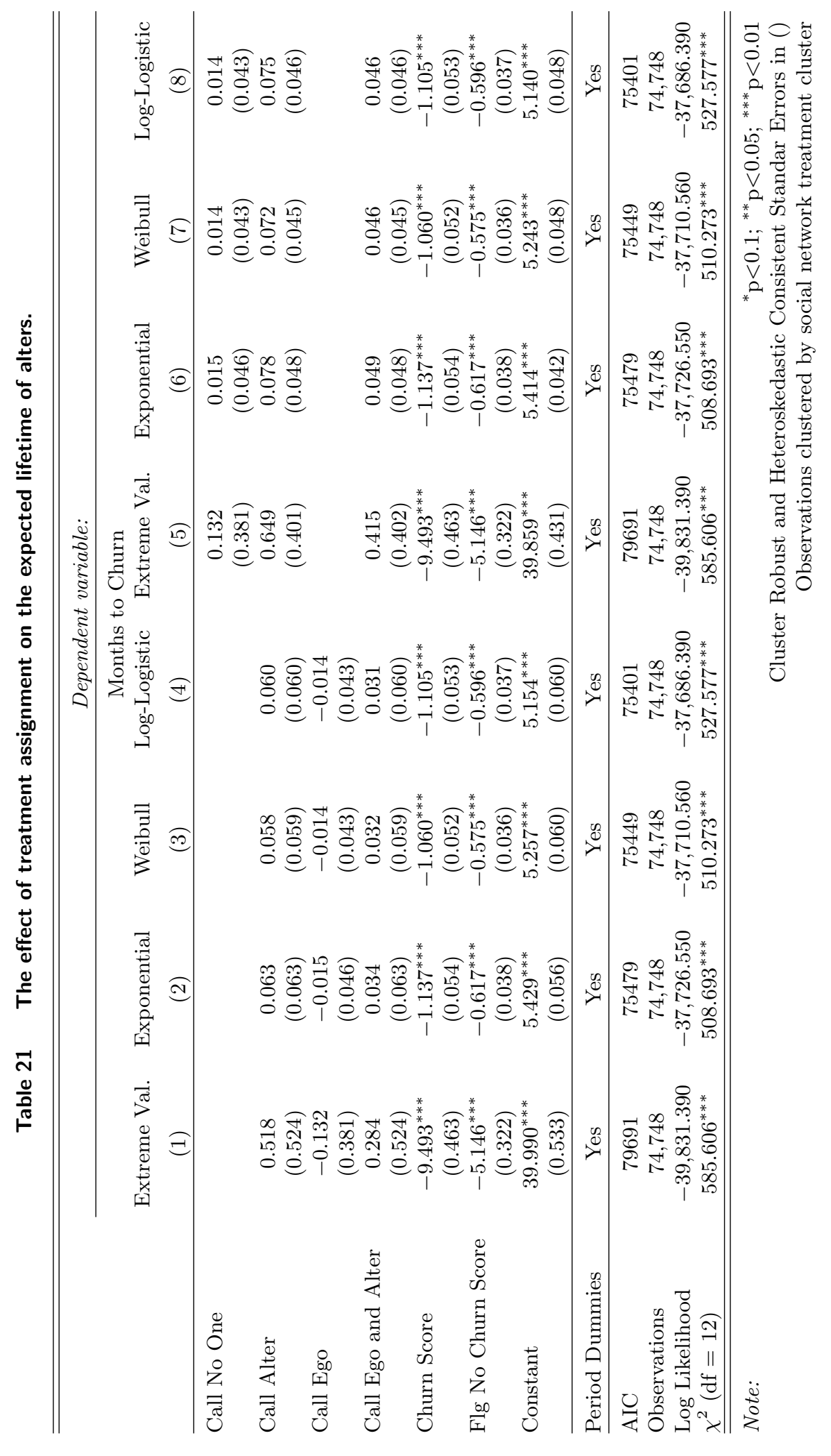


J.1.2. Effect on Expected Customer Lifetime Value Armed with estimates of how treatment assignment affects both monthly bills and expected survival rates we can estimate the CLV of each household. The following are noteworthy features of this exercise: i) we assume that a household generates a constant revenue over time equal to its monthly bill 30 days after treatment assignment; ii) we use TELCO's Weighted Average Cost of Capital of $0.7 \% /$ month, as reported to us by company managers, as our discount rate; iii) we compute CLV over a 10-year horizon (the average TELCO household stays 62 months with the firm). Empirically, we use the following regression:

$$
C L V_{j}=\zeta_{0}+\text { churn_score } \zeta_{j}+\text { treatment }_{\mathbf{j}} \zeta_{\mathbf{2}}+\epsilon_{j}
$$

where $j$ is a unit of analysis in our experiment (an ego, an alter or a cluster), treatment $\mathbf{j}_{\mathbf{j}}$ is a vector of indicators for our treatment conditions and $\zeta_{2}$ is a vector of parameters indicating the corresponding profits for the firm. Differences among these parameters will readily measure the profitability of proactive churn management and of socially-based proactive churn management as described in the beginning of this section. Table 22 shows the results obtained for egos in columns (1) and (2), for alters in columns (3) and (4) and for clusters in columns (5) and (6). Column (1) shows that calling only alters does not change the CLV of egos in our setting. This column also shows that when only egos are listed to be called by the firm their CLV increases by $2.1 \%\left(51.1 /\left(2861-663^{*} 0.7\right)\right)$, where 0.7 is the average churn score across egos in our sample. This is the direct effect of proactive churn management on egos, captured by expression 2 of our framework. This column also shows that when alters are listed to be called by the firm, in addition to egos, the CLV of the latter increases by $6.4 \%\left(154.3 /\left(2861-663^{*} 0.7\right)\right)$. Column $(2)$ shows that the difference between the latter and the former statistics is statistically significant: $4.3 \%$ given by $\left(103.2 /\left(2911-663^{*} 0.7\right)\right)$. This difference is the spillover effect of socially-based proactive churn management on egos, captured by expression 6 in our framework. Columns (3) and (4) show that listing alters to be called by the firm, irrespective of whether egos are called too, does not change their CLV. That is, there are no spillover effects of proactive churn management on alters nor direct effects of socially-based proactive churn management on alters, as captured by expressions 3 and 7 in our framework. Alters are not likely churners in our setting and the results in these columns show that the firm was effective at avoiding extending them good deals. Finally, and for robustness sake, columns (5) and (6) show effects over the clusters used in our experiment. The dependent variable in these regressions is the ratio between the sum of CLV for all egos and alters in the cluster and the number of egos and alters in the cluster, thus adjusting for the fact that different clusters may have different sizes. These columns confirm that in our setting listing likely churners to call is profitable for the firm and more so when their alters are also listed to be called. 


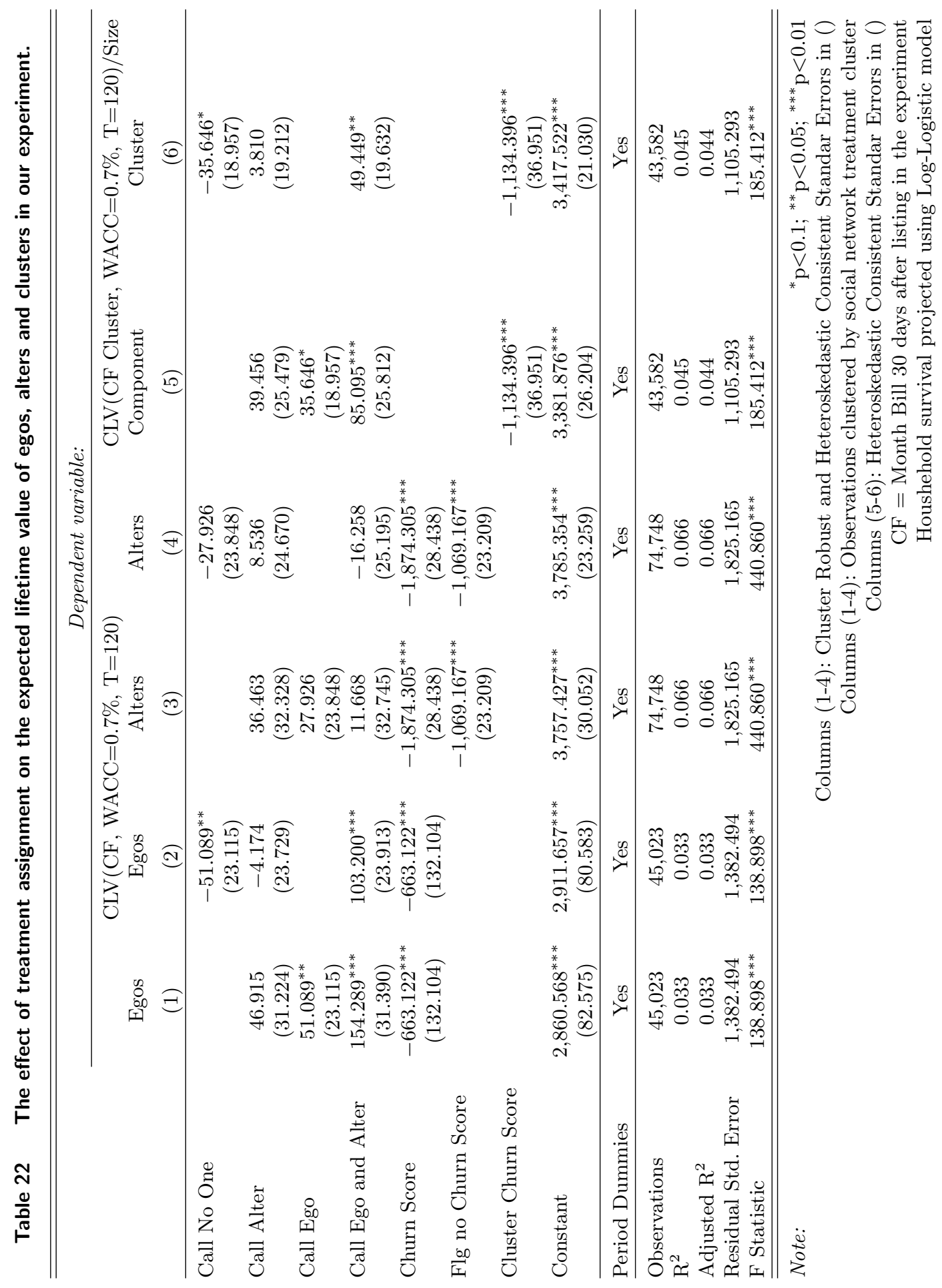

